\title{
Displacement convexity of Boltzmann's entropy characterizes the strong energy condition from general relativity*
}

\author{
Robert J. McCann ${ }^{\dagger}$
}

October 9, 2018

\begin{abstract}
On a Riemannian manifold, lower Ricci curvature bounds are known to be characterized by geodesic convexity properties of various entropies with respect to the Kantorovich-Rubinstein-Wasserstein square distance from optimal transportation. These notions also make sense in a (nonsmooth) metric measure setting, where they have found powerful applications. This article initiates the development of an analogous theory for lower Ricci curvature bounds in timelike directions on a (globally hyperbolic) Lorentzian manifold. In particular, we lift fractional powers of the Lorentz distance (a.k.a. time separation function)
\end{abstract}

*Dedicated to my muse, Carolyn. MSC Primary 53C50, Secondary 49J52 58Z05 83C99 82C35. The author is pleased to thank Spyros Alexakis, Stefanos Aretakis, Eric Carlen, Christian Ketterer and Eric Woolgar for stimulating conversations and relevant references, and to Martin Kell and Stefan Suhr for communicating their work to him in preprint form. He thanks Robert Wald for encouragement, and Elliott Lieb, who drew Boltzmann's entropy to his attention upon hearing about displacement convexity. He is grateful for the hospitality of the University of Chicago, the Mathematical Sciences Research Institute (MSRI) at Berkeley CA, Fields Insitute for the Mathematical Sciences, and Mittag-Leffler Institute during various stages of this work. He acknowledges partial support of his research by Natural Sciences and Engineering Research Council of Canada Grants 21700608 and -15, by a Simons Foundation Fellowship, and by US National Science Foundation Grant No. DMS-144041140 while in residence at MSRI during thematic programs in 2013 and 2016. (C) 2018 by the author.

$\dagger$ Department of Mathematics, University of Toronto, Toronto Ontario M5S 2E4 Canada, mccann@math.toronto.edu 
to probability measures on spacetime, and show the strong energy condition of Hawking and Penrose is equivalent to geodesic convexity of the Boltzmann-Shannon entropy there. This represents a significant first step towards a formulation of the strong energy condition and exploration of its consequences in nonsmooth spacetimes, and hints at new connections linking the theory of gravity to the second law of thermodynamics.

\section{Contents}

1 Introduction 2

1.1 Optimal transport with Lorentz distances . . . . . . . . . . 4

1.2 Main results and discussion . . . . . . . . . . . . . . . . . . . . . . . . . . .

1.3 Further related works . . . . . . . . . . . . . . 11

1.4 Plan of the paper ................ . . . . 12

2 Geodesics of probability measures on spacetime 13

3 Lagrangian, Hamiltonian, Lorentz distance 19

4 Kantorovich duality with Lorentz distance 29

5 Characterizing $q$-geodesics via duality 34

6 Entropic convexity from Ricci lower bounds 43

7 Relaxing separation from the null future 51

8 Ricci lower bounds from entropic convexity 59

A Monge-Mather shortening estimate 63

\section{Introduction}

The second law of thermodynamics is amongst the most remarkable and universal laws in all of physics. It asserts that for an isolated (or adiabatic) system, only certain physical processes are permitted. Moreover the distinction between permitted and forbidden processes is determined by the prin- 
cipal that the associated entropy be non-decreasing in time. In other words, these dynamical processes exhibit a preferred direction of time. There is an analogous law governing the dynamics of black holes in general relativity, Einstein's theory of gravity, which states that the area of the event horizon of a black hole is proportional to its entropy, hence can only increase [21] [18]. In fact, this idea can also be turned on its head, with the postulated proportionality used to derive general relativity [54] and other forms of gravity [90] [91] as emergent, entropic (i.e. statistical) forces.

In this paper we investigate another, quite different law concerning the information-theoretic entropy of probability measures on spacetime. Using the $q$-Lorentz-Wasserstein distance from optimal transportation [38] in place of a metric on such measures, we claim convexity of this entropy along the geodesics of probability measures which result is equivalent to the strong energy condition of Hawking and Penrose [79] [51] [53], which - despite its more limited range of validity than the dominant energy condition, e.g. [31] - plays an important role in gravitational theory.

The strong energy condition is a positive-definiteness condition on the stress-energy tensor $T_{a b}$, which encodes the energy and momenta densities and fluxes at each point in spacetime. It asserts that in each normalized timelike direction $v^{a}$, this tensor dominates half its trace: $T_{a b} v^{a} v^{b} \geq \frac{1}{2} T$. Equivalently, since the Einstein equation postulates proportionality of $T_{a b}-\frac{1}{2} T g_{a b}$ to the Ricci tensor $\mathrm{Ric}_{a b}$, in the absence of cosmological constant the strong energy condition boils down to Ricci non-negativity in timelike directions: $\operatorname{Ric}_{a b} v^{a} v^{b} \geq 0$. In the presence of trapped or other distinguished surfaces, it implies a spacetime must have singularities, e.g. [52] [93] [89]. It is also understood to be responsible for the empirical fact that gravity is purely attractive, and never repulsive (at least, in the absence of rotation; c.f. [31] and Raychaudhuri's equation).

That the Hawking and Penrose condition has anything to do with entropy or the second law of thermodynamics may seem surprising. However, this relationship is foreshadowed by recent developments in Riemannian geometry, the mathematical progenitor of general relativity. There a line of research due to the author [66] and his collaborators [36] 37], Otto and Villani [78, and von Renesse and Sturm, has culminated in a characterization of Riccicurvature lower bounds involving only the displacement convexity of certain information-theoretic entropies [80]. This in turn led Sturm [85] and independently Lott and Villani [62] to the development of a robust theory for lower Ricci curvature bounds in a (non-smooth) metric-measure space setting. A 
vibrant theory of such spaces has emerged rapidly since that time, which would take us too far afield to survey; see e.g. [7] [8] [10] [11] [12] 33] [34] [35] [39] [47] [49] [57] [70] [76]; competeing approaches to the complementary upper bounds may be found in [72] [50] and [86] [87].

Our purpose is to initiate the development of an analogous theory in the equally tantalizing and more physically relevant setting of gravitation. A particular consequence of our theory is that it becomes possible to define what it means for a volume measure on a (nonsmooth) Lorentzian geodesic space [58] to satisfy the strong energy condition, and to show that many familiar implications of this condition in the smooth setting extend to the nonsmooth geometries representing super-solutions of the vaccuum Einstein equations. This is particularly relevant to gravitational theory since a wide variety of smooth spacetimes contain timelike geodesics which are neither extendible nor complete [52]. However, the present manuscript is devoted to showing consistency of the proposed definition with the classical one in the smooth setting, and defers the development of a theory of non-smooth spacetimes to a forthcoming work.

From the technical point of view, our major innovations include the introduction of the strictly convex Lagrangian (20) inducing the fractional time-separation function $\ell(x, y)^{q}$ for $0<q<1$, and the development of techniques for resolving the resulting optimal transportation problem on spacetime posed by Eckstein and Miller [38 and its dual, despite the singularities

of these objective functionals. Even for the semi-relativistic Lagrangian of Brenier [29] (corresponding to $q=1$ on Minkowski hyperplanes), the challenges such singularities present have plagued researchers for more than a decade, and stimulated a series of works [69] [27] 26] 88] leading up to a preprint by Kell and Suhr [56] which, for $q=1$, resolves certain analogous issues described below simultaneously and independently of the present manuscript.

\subsection{Optimal transport with Lorentz distances}

Let $\left(M^{n}, g\right)$ be a smooth, connected, Hausdorff, time-oriented Lorentzian manifold, with a signature $(+,-, \ldots,-)$ metric. It follows from results of Nomizu, Ozeki [73] and Geroch [45] that $M$ is second countable and that its topology also arises from a complete Riemannian metric $\tilde{g}$. Hereafter these hypotheses may be abbreviated by saying $M$ is a spacetime. A tangent vector $v \in T_{x} M$ is said to be timelike $(>0)$, spacelike $(<0)$ or null $(=0)$, according 
to the sign of $v^{a} g_{a b} v^{b}$. The time-orientation of the manifold allows causal (i.e. non-spacelike) vectors to be classified continuously as either future- or pastdirected, with $v \neq 0$ being future-directed if and only if $-v$ is past-directed.

For $q \in(0,1]$, define the convex Lagrangian $L(v, x ; q):=-\left(g_{a b}(x) v^{a} v^{b}\right)^{q / 2} / q$ on the tangent bundle of $M$, with the convention that $L(v, x ; q)=+\infty$ unless $v$ is future-directed. For curves $\sigma \in C^{0,1}([0,1] ; M)$, the associated action is

$$
A[\sigma ; q]:=\int_{0}^{1} L\left(\sigma^{\prime}(s), \sigma(s) ; q\right) d s .
$$

We define the (q-dependent) Lorentz distance between any two points as the infimum

$$
\ell(x, y ; q)=-\inf \left\{A[\sigma ; q] \mid \sigma \in C^{0,1}([0,1] ; M), \sigma(0)=x, \sigma(1)=y\right\}
$$

over Lipschitz curves with fixed endpoints. For notational simplicity, we adopt the convention

$$
(-\infty)^{1 / q}:=-\infty=:(-\infty)^{q}
$$

throughout. With this convention, $(q \ell(x, y, q))^{1 / q}=: \ell(x, y)$ is independent of $q \in(0,1]$ and satisfies the reverse triangle inequality

$$
\ell(x, y) \geq \ell(x, z)+\ell(z, y)
$$

it represents the maximum amount that a physical particle can age while travelling from $x$ to $y$, and is therefore also known as the time-separation function. It differs from the textbook definitions [52] [77] [19] of the Lorentz distance, which is non-negative, only in that $\ell(x, y)=-\infty$ if there is no future-directed curve from $x$ to $y$. This has the convenient consequence that $y$ lies in the causal future of $x$ if and only if $\ell(x, y) \geq 0$, and in the chronological future of $x$ if and only if $\ell(x, y)>0$, (which one may also take as definitions of the italicized terms). It is also important for ensuring that whenever possible, the solutions to the transportation problem introduced in the next paragraphs couple only causally related events.

We henceforth assume our spacetimes $(M, g)$ to be globally hyperbolic, meaning $M$ has no closed causal curves, and for each $x, y \in M$ the intersection

$$
J^{+}(x) \cap J^{-}(y):=\{z \in M \mid \ell(x, z) \geq 0\} \cap\{z \in M \mid \ell(z, y) \geq 0\}
$$


of the causal future of $x$ with the causal past of $y$ is compact [23]. These assumptions ensure that the infimum (2) is actually attained [13] 84]; Jensen's inequality ensures the curve that attains it is independent of $q$. When $\ell(x, y)>0$ any such curve is a geodesic; moreover this geodesic is affinely parameterized if $q<1$, in which case we call it an action minimizing (or proper-time maximizing) segment. Each point $z$ on this segment saturates the triangle inequality (4); conversely, when $\ell(x, y)>0$, inequality (44) holds strictly unless $z$ lies on an action minimizing segment joining $x$ to $y$.

Let $\mathcal{P}(M)$ be the set of Borel probability measures on $M$, and $\mathcal{P}_{c}(M):=$ $\{\mu \in \mathcal{P}(M) \mid$ spt $\mu$ is compact $\}$, where spt $\mu$ denotes the smallest closed subset of $M$ carrying the full mass of $\mu \geq 0$. We lift the Lorentz distance $\ell$ from $M$ to $\mathcal{P}(M)$ as follows: given $\mu$ and $\nu$ on $M$, we recall the $q$-LorentzWasserstein distance introduced independently from the present manuscript by Eckstein and Miller [38],

$$
\ell_{q}(\mu, \nu):=\sup _{\pi \in \Pi_{\preceq}(\mu, \nu)}\left(\int_{M \times M} \ell(x, y)^{q} d \pi(x, y)\right)^{1 / q}
$$

where $\Pi_{\preceq}(\mu, \nu)$ denotes the of joint measures $\pi \geq 0$ on $M^{2}$ with spt $\pi \subset$ $\ell^{-1}([0, \infty])$ and having $\mu$ and $\nu$ for marginals. When $q=1$ it represents the maximum expected proper-time which can elapse between the distributions of events represented by $\mu$ and those represented by $\nu$. If $\ell$ is dominated by a lower semicontinuous function in $L^{1}(d \mu) \oplus L^{1}(d \nu)$ - as when $\mu$ and $\nu$ are compactly supported - then (6) is attained, e.g. 88, [92, since $\ell$ is upper semi-continuous, $d_{\tilde{g}}$ makes $M$ into a complete separable metric space, and our convention (3) ensures the value of the supremum (6) is unchanged if we replace $\Pi_{\preceq}(\mu, \nu)$ by the set $\Pi(\mu, \nu)$ of all measures on $M^{2}$ having $\mu$ and $\nu$ for marginals. If in additition $\Pi_{\preceq}(\mu, \nu)$ is non-empty then (6) is finite; c.f. [38]. The maximizing $\pi$ will be called $\ell^{q}$-optimal, since $\ell(x, y ; q)=\frac{1}{q} \ell(x, y)^{q}$. Our convention that $\ell_{q}(\mu, \nu)=-\infty$ unless there exists a pairing $\pi \in \Pi(\mu, \nu)$ with $y$ lying in the causal future of $x$ for $\pi$-a.e. $(x, y)$ is at variance with the convention of Eckstein and Miller (who instead define the $q$-Lorentz Wasserstein distance to be zero in this case). Nevertheless, the reverse triangle inequality (41) implies [38]

$$
\ell_{q}(\mu, \nu) \geq \ell_{q}(\mu, \lambda)+\ell_{q}(\lambda, \nu)
$$

for all $\lambda, \mu, \nu \in \mathcal{P}(M)$, where we use the convention $\infty-\infty=-\infty$ to interpret the right-hand side of (7). This precisely parallels the fact that the 
Kantorovich-Rubinstein-Wasserstein distances $W_{p}$ in the metric space setting described e.g. in [92, Definition 6.1] satisfy the usual triangle inequality. Continuing this analogy allows us to lift the notion of maximizing geodesic segment from points to (chronologically sequenced) probability measures:

Definition 1.1 (Geodesics of probability measures on spacetime) $W e$ say $s \in[0,1] \mapsto \mu_{s} \in \mathcal{P}(M)$ is a q-geodesic if and only if

$$
\ell_{q}\left(\mu_{s}, \mu_{t}\right)=(t-s) \ell_{q}\left(\mu_{0}, \mu_{1}\right) \in(0, \infty)
$$

for each $0 \leq s<t \leq 1$.

With this terminology, $q$-geodesics are implicitly future-directed and timelike. Subsequent sections establish the existence of $q$-geodesics connecting fairly arbitrary endpoints $\mu_{0}$ and $\mu_{1}$. The interpolating measures $\mu_{s}$ turn out to inherit compact support from the endpoints. Let $\mathcal{P}^{a c}(M) \subset \mathcal{P}(M)$ denote the set of measures $\mu$ which are absolutely continuous with respect to the Lorentzian volume $\operatorname{vol}_{g}$, and $\mathcal{P}_{c}^{a c}(M):=\mathcal{P}^{a c}(M) \cap \mathcal{P}_{c}(M)$. If, in addition, $\mu_{1}$ or $\mu_{0} \in \mathcal{P}_{c}^{a c}(M)$ and $q<1$, we show the $q$-geodesic joining them to be unique under the technical restriction of $q$-separation proposed in Definition 4.1. which amounts essentially to the positivity of $\ell$ throughout the supports of all $\ell^{q}$-optimal $\pi \in \Pi\left(\mu_{0}, \mu_{1}\right)$. This implies in particular that all $\ell^{q}$-optimal $\pi$ correlate the endpoint distributions of events chronologically, so that $x$ lies in the chronological past of $y$ whenever $(x, y) \in \operatorname{spt} \pi$. Apart from the second endpoint, the whole $q$-geodesic lies in $\mathcal{P}_{c}^{a c}(M)$ in this case. We define the relative entropy $E_{V}$ and Boltzmann-Shannon entropy $E_{0}$ on $\mathcal{P}^{a c}(M)$ :

Definition 1.2 (Entropy) Given $V \in C^{2}(M)$ and $\mu \in \mathcal{P}^{a c}(M)$ having density $\rho:=d \mu / d m$ with respect to the weighted Lorentzian volume $d m=$ $e^{-V} d \mathrm{vol}_{g}$, we define

$$
E_{V}(\mu):=\int_{M} \rho(x) \log \rho(x) e^{-V(x)} d v o l_{g}(x)
$$

if the integral has a well-defined value in $[-\infty, \infty]$, and set $E_{V}(\mu):=-\infty$ otherwise. When $\mu$ vanishes outside a set $U \subset M$ of finite volume $\operatorname{vol}_{g}[U]<$ $\infty$, as when $\mu$ is compactly supported, Jensen's inequality shows

$$
E_{V}(\mu) \geq-\log \int_{U} e^{-V(x)} d \operatorname{vol}_{g}(x)>-\infty
$$


One can define $E_{V}(\mu)=+\infty$ if $\mu \in \mathcal{P}(M) \backslash \mathcal{P}^{a c}(M)$. When $V:=0$ the Boltzmann-Shannon entropy $E_{0}$ results (but with sign differing from the usual convention of the physics literature).

Our central results (Theorems 6.4 and 8.5) are foreshadowed by the following corollary, which characterizes the strong energy condition of Hawking and Penrose [53] via the convexity of Boltzmann-Shannon entropy along $q$ geodesics in $\mathcal{P}_{c}^{a c}(M)$. It incorporates the possibility of non-vanishing cosmological constant $K \geq 0$. To avoid technical complications associated with the lack of smoothness of the Lorentz distance $\ell(x, y)$ at points where it vanishes, we prefer to focus our attention on geodesics whose endpoints $\mu_{0}$ and $\mu_{1}$ are totally chronologically related in the sense that $\operatorname{spt}\left[\mu_{0} \times \mu_{1}\right] \subset\{\ell>0\}$; i.e. each point $y \in \operatorname{spt} \mu_{1}$, lies in the timelike future of each point in spt $\mu_{0} \subset M$, as in the following corollary. Unfortunately, this cannot remain true for $\operatorname{spt}\left[\mu_{s} \times \mu_{t}\right]$ when $t-s>0$ is small, which is more delicate yet apparently unavoidable. We resolve this difficulty by showing the aforementioned $q$-separation propagates from the endpoints to the interior of a $q$-geodesic.

Corollary 1.3 (Positive energy = entropic displacement concavity) Let $\left(M^{n}, g\right)$ be a globally hyperbolic spacetime. Fix $0<q<1$. (i) If the Lorentzian metric satisfies $\operatorname{Ric}_{a b} v^{a} v^{b}<K \in \mathbf{R}$ in some timelike direction $(v, x) \in T M$ normalized so that $g_{a b} v^{a} v^{b}=1$, then a q-geodesic $s \in[0,1] \mapsto \mu_{s} \in \mathcal{P}_{c}^{a c}(M)$ exists along which $e(s):=E_{0}\left(\mu_{s}\right)$ is $C^{2}$-smooth and satisfies $e^{\prime \prime}(0)<K \ell_{q}\left(\mu_{0}, \mu_{1}\right)^{2}$; moreover, $\operatorname{spt}\left[\mu_{0} \times \mu_{1}\right]$ is disjoint from $\{\ell \leq 0\}$ and can be chosen to be contained in any specified neighbourhood of $(x, x)$. (ii) Conversely, if the metric tensor satisfies $\operatorname{Ric}_{a b} v^{a} v^{b} \geq K v^{a} v^{b} g_{a b} \geq 0$ in all timelike directions $(v, x) \in T M$ of the tangent bundle, then $e^{\prime \prime}(s) \geq$ $\frac{1}{n} e^{\prime}(s)^{2}+K \ell_{q}\left(\mu_{0}, \mu_{1}\right)^{2}$ holds along all q-geodesics $s \in[0,1] \mapsto \mu_{s} \in \mathcal{P}_{c}^{a c}(M)$ with finite entropy endpoints and $\operatorname{spt}\left[\mu_{0} \times \mu_{1}\right]$ disjoint from $\{\ell \leq 0\}$. Here $e^{\prime \prime}(s)$ is interpreted distributionally.

Apart from possible aesthetic or philosophical considerations, the advantage of the reformulation of the strong energy condition provided by this corollary is that the notions it relies on - namely, q-geodesics, entropy, and convexity - require only a time-separation function $\ell(x, y)$ (which determines the causal structure), and a reference measure $m$ (given in this case by $\left.d m=e^{-V} d \mathrm{vol}_{g}\right)$. As a result, they can be adapted to non-smooth settings, including the Lorentzian geodesic spaces of Kunzinger and Sämann [58], where 
they offer a promising approach to the development of a synthetic theory of spaces which enjoy uniform lower Ricci curvature bounds in all timelike directions. We call such spaces $T C D_{q}^{e}(K, N)$ spaces, in analogy with the corresponding theory of curvature dimension conditions in metric-measure spaces pioneered by Lott, Villani [62] and Sturm [85]. Here the superscript $e$ refers to the simpler alternative but equivalent definition of these conditions by Erbar, Kuwada and Sturm [39]; the possibility $q \neq 2$ was explored in the metric-measure setting by Kell [55] for $q \geq 1$, and the leading $\mathrm{T}$ is a mnemonic for timelike, following the terminology used by Woolgar and Wylie in their work on singularities and splitting theorems for $N$-Bakry-Émery spacetimes 94. We develop such a theory for nonsmooth spacetimes in a forthcoming work.

\subsection{Main results and discussion}

Our main result is considerably more general than the corollary indicated above. It concerns lower bounds for the following modified version of the Ricci tensor, called the $N$-Bakry-Émery-Ricci tensor in honor of [15]:

Definition 1.4 ( $N$-Bakry-Émery-Ricci tensor) Given $n \neq N \in[-\infty, \infty]$ and $V \in C^{2}(M)$ on a Lorentzian manifold $\left(M^{n}, g\right)$, we define the modified Ricci tensor by

$$
\operatorname{Ric}_{a b}^{(N, V)}:=\operatorname{Ric}_{a b}+\nabla_{a} \nabla_{b} V-\frac{1}{N-n}\left(\nabla_{a} V\right)\left(\nabla_{b} V\right)
$$

and adopt the conventions $\operatorname{Ric}_{a b}^{(n, V)}:=\operatorname{Ric}_{a b}$ and $V=0$ if $N=n$.

Explored in the Lorentzian context by Case [32, it was also used by Woolgar and collaborators (see [94] and the references there) to extend Hawking and Penrose type singularity theorems to manifolds-with-density — on which the Lorentzian volume $d \operatorname{vol}_{g}(x)$ is replaced by $d m(x):=e^{-V(x)} d \operatorname{vol}_{g}(x)$.

For fixed $K \geq 0, N \geq n, V \in C^{2}(M)$ and $0<q<1$, the results of Corollary 6.6 and Theorem 8.5 below show that

$$
\operatorname{Ric}_{a b}^{(N, V)} v^{a} v^{b} \geq K g_{a b} v^{a} v^{b}
$$

holds for each timelike vector $(v, x) \in T M$ tangent to a globally hyperbolic spacetime $\left(M^{n}, g\right)$ if and only if the distributional second-derivative of the 
relative entropy $e(s):=E_{V}\left(\mu_{s}\right)$ satisfies

$$
e^{\prime \prime}(s) \geq \frac{1}{N} e^{\prime}(s)^{2}+K \ell_{q}\left(\mu_{0}, \mu_{1}\right)^{2}
$$

on each $q$-geodesic $s \in[0,1] \mapsto \mu_{s} \in \mathcal{P}_{c}^{a c}(M)$ with $q$-separated, finite entropy endpoints. The requirement that each pair of endpoints be compactly supported and $q$-separated can be relaxed if we are content to have weak displacement convexity, meaning the existence of a single $q$-geodesic joining them which satisfies the required inequalities; see Corollary 7.5. This is the Lorentzian analog of Erbar, Kuwada and Sturm's reformulation $C D^{e}(K, N)$ [39] of Sturm's original curvature-dimension condition $C D(K, N)$ [85] for a metric measure space $(M, d, m)$ (also formulated independently, for $K / N=$ 0 , by Lott and Villani [62]); as long as geodesics in $M$ are essentially nonbranching the two formulations are shown to be equivalent by combining the results of Bacher and Sturm [14] and Cavalletti and Milman [33] with those of 39 .

Several points deserve further mention. First, the coefficient $\ell_{q}\left(\mu_{0}, \mu_{1}\right)^{2}$ of $K$ is natural, in the sense that it disappears from (13) if we 'arc-length' reparameterize the $q$-geodesic $\left(\mu_{s}\right)_{s \in[0,1]}$ over $\left[0, \ell_{q}\left(\mu_{0}, \mu_{1}\right)\right]$ instead of $[0,1]$. Second, in contradistinction to theories of Lott-Villani and Sturm, our theory does not encompass negative lower Ricci curvature bounds: although (13) continues to imply (12) when $K<0$, by way of converse we can only deduce that (12) implies

$$
e^{\prime \prime}(s) \geq \frac{1}{N} e^{\prime}(s)^{2}+K \int_{M \times M} \ell(x, y)^{2} d \pi(x, y)
$$

where $\pi \in \Pi\left(\mu_{0}, \mu_{1}\right)$ is $\ell^{q}$-optimal. To obtain (13) from this using Jensen's inequality requires $K \geq 0$, as in Remark 6.7 below.

In the present smooth context, its equivalence to (12) shows independence of (13) on $q \in(0,1)$. It is not clear whether this $q$-independence extends to the non-smooth $T C D_{q}^{e}(K, N)$ spaces of our sequel. In the metric-measure context, the analogous class of spaces are those satisfying the $C D_{q}(K, N)$ condition defined using the $q$-Wasserstein metric by Kell for $q \geq 1$ [55]. It is an interesting question whether the class of spaces which satisfy his condition actually depends on $q$; under suitable restrictions such as $1<q \leq 2$ and $K \geq 0$ the subset of Riemannian manifolds-with-density (also called smooth metric measure spaces) which satisfy it does not. 
Finally, the astute reader will note we have established convexity, rather than the monotonicity which would be required of the thermodynamic entropy by the second law. But our entropy is not the thermodynamic entropy, and the extent to which there is a connection, if any, between them remains mysterious.

\subsection{Further related works}

The need to extend concepts from Lorentzian geometry to non-smooth settings is discussed, e.g., in [58]. The idea of approaching this problem through displacement convexity of the entropy on the space of probability measures is inspired by its success in the Riemannian context following [62 [85].

Optimal transportation with respect to Lagrangians which are smooth and strictly convex is laid out in Villani [92, following works of Benamou, Brenier [22] 29], Bernard and Buffoni [24] [25]. As for the square distance [66] [36], these initial investigations established existence, uniqueness and regularity of optimal maps and interpolants $\mu_{s}$. Ohta [74] [75], Lee [59], Kell [55, and Schachter [83, continued this line of research by exploring entropic displacement convexity and its relation to notions of curvature for Lagrangians in varying degrees of generality, always assuming smoothness of $L$ except perhaps at the zero vector. Relatively little attention has been devoted to singular Lagrangians, apart from the subRiemannian case [5] [1] [42] 60] [16].

The most notable exceptions appear in work of Eckstein and Miller [38], who introduced the $q$-Lorentz Wasserstein distance (2) as a means of exploring causality relations between spacetime probability measures independently of the present manuscript, and Suhr [88], who studied the $q=1$ maximization problem (6) along with various generalizations complementary to ours, and focused especially on measures $\mu$ and $\nu$ which, instead of being absolutely continuous with respect to $d \mathrm{vol}_{g}$, are supported on spacelike hypersurfaces. His manuscript, which we learned of only during the writing of this work, provides analogs to several of our results from sections 2 and 5 in this rather different context. As antecedents for his study he cites the cosmic initial velocity reconstruction problem addressed by Frisch et al [43] 30], and the work of Bertrand and Puel [27] on Brenier's relativistic heat equation [29], which involves the special case of Suhr's problem set on parallel planes in Minkowski space (and was also explored in [69] [26]). The enhancing effect of Newtonian self-gravity on the displacement convexity of various entropies 
was first discovered by Loeper [61].

After the present results had been announced, we learned of work of Kell and Suhr which, particularly for $q=1$, develops a duality theory analogous to that of $\S 4$, under hypotheses which are related to but different from our $q$-separation; their conditions are phrased in terms of the existence of dynamical transport plans (= measures on action minimizing segments) which need not a priori be optimal, but whose velocities are locally bounded away from the light cone [56]. They also address the absolute continuity of 1-geodesics, using an approach different from both Corollary 5.9 and Remark [6.2, and indicate possible extensions to $q<1$. We similarly learned of a heuristic argument by Gomes and Senici [48] extending displacement convexity to planning problems from mean-field games which involve rather general smooth convex Hamiltonians and, strikingly, incorporate local congestion effects.

\subsection{Plan of the paper}

The plan of the paper is the following. In the next section we establish the existence and uniqueness of $q$-geodesics connecting chronologically related probability measures on spacetime. It is followed by a section which recalls various notions from non-smooth analysis, and lays out needed properties of the Lorentz distance $\ell(x, y ; q)$ and the $q$-dependent family of Lagrangians and Hamiltonians which define it. In Section \$4 we develop a KantorovichKoopmans duality theory for the optimal transportation problem ([6), under the aforementioned restriction that the probability measures $\mu$ and $\nu$ be $q$ separated. This duality theory allows us to develop a Lagrangian calculus for $q$-geodesics in $\$ 5$, based on the existence and uniqueness of optimal maps. The proof that intermediate-time maps have Lipschitz inverses is relegated to Appendix A, see also Suhr for $q=1$ [88]. In \$6 this calculus is employed to compute derivatives of the entropy along $q$-geodesics and establish our claim that Ricci non-negativity in timelike directions implies entropic displacement convexity — at least along geodesics with $q$-separated endpoints. Section $\$ 7$ shows this $q$-separation restriction can relaxed if we are content to conclude weak displacement convexity of the entropy. In this section we also extend our results concerning existence and uniqueness of optimal maps to situations where it is unclear whether strong duality is attained. The converse implication, that weak entropic displacement convexity implies timelike Ricci non-negativity, is established in Section $\$ 8$. 


\section{Geodesics of probability measures on space- time}

The main goal of this section is to derive conditions which guarantee the existence and uniqueness of $q$-geodesics in $\mathcal{P}(M)$. A more thorough characterization of their properties relies on the development of a strong duality theory, both of which require additional hypotheses and are deferred to subsequent sections. See also Suhr for the special case $q=1$ [88.

We begin by introducing the singular set $\operatorname{sing}(\ell)$ of the Lorentz distance, which consists of the timelike cut locus of $M$ together with all pairs of points not in chronological sequence. It is well-known to be closed, and can also be characterized as the set where $\ell$ fails to be smooth; see Theorem 3.6.

Definition 2.1 (Singularities of the Lorentz distance) Let $\left(M^{n}, g\right)$ be a globally hyperbolic spacetime. We say $(x, y) \in \operatorname{sing}(\ell)$ unless $\ell(x, y)>0$ and $x$ and $y$ both lie in the relative interior of some affinely parameterized proper-time maximizing geodesic segment.

We also make frequent use of the following construction familiar from optimal transportation.

Definition 2.2 (Push-forward) Given a Borel map $F: M \longrightarrow N$ between two metric spaces, and a Borel measure $\mu \geq 0$ on $M$, we define the pushforward $F_{\#} \mu$ to be the Borel measure on $N$ given by $F_{\#} \mu(V)=\mu\left(F^{-1}(V)\right)$ for each $V \subset N$.

Global hyperbolicity is used to ensure the interpolating measures $\left(\mu_{s}\right)_{s \in[0,1]}$ which make up each $q$-geodesic inherit compact support from the endpoints $\mu_{0}$ and $\mu_{1}$. It also ensures various familiar properties of the Lorentz distance $\ell(x, y)$ recalled for the reader's convenience in the next two lemmas.

Lemma 2.3 (Semicontinuity of Lorentz distance) Let $\left(M^{n}, g\right)$ be a globally hyperbolic spacetime. The Lorentz distance $\ell: M^{2} \longrightarrow[0, \infty) \cup\{-\infty\}$ defined by $q=1$ in (2) is (a) upper semicontinuous on $M \times M$, (b) continuous on $\ell^{-1}([0, \infty))$ and $(c)$ smooth precisely on the complement of the closed set $\operatorname{sing}(\ell)$. 
Proof. Continuity of the function $\ell_{+}:=\max \{\ell, 0\}$ is well known [19, Corollary 4.7] [77, Lemma 14.21-22]. Claims (a)-(b) follow immediately since $\ell^{-1}([c, \infty])=\ell_{+}^{-1}\left(\left[c_{+}, \infty\right]\right)$ is closed for each $c \in \mathbf{R}$ with $c_{+}=\max \{c, 0\}$.

The proof of (c) is deferred to Theorem 3.6 below; see also Proposition 9.29 of [19].

Lemma 2.4 (Midpoint continuity away from cut locus) For each $s \in$ $[0,1]$ and $(x, y) \in M \times M \backslash \operatorname{sing}(\ell)$ there is a unique $z=z_{s}(x, y) \in M$ such that

$$
\ell(x, z)=s \ell(x, y) \text { and } \ell(z, y)=(1-s) \ell(x, y) .
$$

Moreover, $z$ depends smoothly on $(s, x, y) \in[0,1] \times(M \times M \backslash \operatorname{sing}(\ell))$.

Proof. Let $(\bar{x}, \bar{y}) \in M \times M \backslash \operatorname{sing}(\ell)$. The definition of $\operatorname{sing}(\ell)$ implies both $\bar{x}$ and $\bar{y}$ lie in the relative interior of some timelike action minimizing segment $s \in[0,1] \mapsto \sigma(s)$, and $\bar{y}$ lies in the chronological future of $\bar{x}$. Thus $\bar{y}$ is strictly within the timelike cut locus of $\bar{x}$ which means (i) that there is a unique proper-time parameterized action minimizing geodesic $z_{s}(\bar{x}, \bar{y})$ joining $\bar{x}$ to $\bar{y}$ (e.g. Corollary 9.4 of [19]), hence a unique solution to (15), (ii) it is given by $z_{s}(\bar{x}, \bar{y})=\exp _{\bar{x}} s \bar{v}$ for some $\bar{v}=\bar{v}(\bar{x}, \bar{y}) \in T_{x} M$, and (iii) $\bar{x}$ and $\bar{y}$ are non-conjugate, so the smooth map $(x, v) \in T M \mapsto \exp _{x} v \in M \times M$ acts diffeomorphically on a neighbourhood of $(\bar{x}, \bar{v})$. Thus $\bar{v}(x, y)$ depends smoothly on $(x, y)$ near $(\bar{x}, \bar{y})$, which implies $z_{s}(x, y)$ is smooth outside the closed set $\operatorname{sing}(\ell)$ of Lemma 2.3.

The preceding and follows lemmas establish the interpolating point $z_{s}(x, y)$ and set $Z_{s}(\cdot)$ notations used throughout.

Lemma 2.5 (Midpoint sets inherit compactness) Fix a globally hyperbolic spacetime $(M, g)$. Given $S \subset M \times M$ and $s \in[0,1]$ let

$$
\begin{aligned}
Z_{s}(S) & :=\bigcup_{(x, y) \in S} Z_{s}(x, y) \quad \text { where } \\
Z_{s}(x, y) & :=\{z \in M \mid \ell(x, z)=\operatorname{s\ell }(x, y) \text { and } \ell(z, y)=(1-s) \ell(x, y)\} 17)
\end{aligned}
$$

if $\ell(x, y) \geq 0$ and $Z_{s}(x, y):=\emptyset$ otherwise. If $S$ is precompact then $Z(S):=$ $\cup_{s \in[0,1]} Z_{s}(S)$ is precompact. If, in addition, $S$ is compact then $Z(S)$ and $Z_{s}(S)$ are compact. 
Proof. Since $Z_{s}(x, y):=\emptyset$ unless $\ell(x, y) \geq 0$ and Lemma 2.3 implies $\{\ell \geq 0\}$ is closed, it costs no generality to restrict our attention to precompact sets $S \subset\{\ell \geq 0\}$. For such a set, fix an arbitrary sequence $\left\{z_{k}\right\}_{k=1}^{\infty}$ in $Z(S)$. Then there are a sequence of times $s_{k} \in[0,1]$ and timelike action minimizing geodesic segments $\sigma_{k}:[0,1] \longrightarrow M$ with endpoints $\left(x_{k}, y_{k}\right):=\left(\sigma_{k}(0), \sigma_{k}(1)\right)$ in $S$ such that $\sigma_{k}\left(s_{k}\right)=z_{k}$. Precompactness of $S$ yields a subsequential limit $\left(x_{\infty}, y_{\infty}\right)=\lim _{j \rightarrow \infty}\left(x_{k(j)}, y_{k(j)}\right)$ for the endpoints. Corollary 3.32 of [19] yields a future-directed limit curve $\sigma_{\infty}$ of this subsequence which joins $x_{\infty}$ to $y_{\infty}$. From its Arzelà-Ascoli based proof, we see more is true: letting $\tilde{\sigma}_{k}$ denote the reparameterization of $\sigma_{k}$ with respect to its arclength for the Riemannian metric $\tilde{g}$ we have uniform convergence of $\tilde{\sigma}_{k(j)}$ to $\tilde{\sigma}_{\infty}$; moreover this sequence of curves has arclength bounded by $c$ independent of $k$. For each $k$ there exists $c_{k} \leq c$ such that $z_{k}=\sigma_{k}\left(s_{k}\right)=\tilde{\sigma}_{k}\left(c_{k}\right)$. Extracting a further subsequence without relabelling yields a limit $c_{\infty}=\lim _{j \rightarrow \infty} c_{k(j)}$. Uniform convergence of the 1-Lipschitz curves $\tilde{\sigma}_{k(j)}$ then gives $\tilde{\sigma}_{\infty}\left(c_{\infty}\right)=\lim _{j \rightarrow \infty} \tilde{\sigma}_{k(j)}\left(c_{k(j)}\right)$ to establish the desired subsequential limit of $\left\{z_{k}\right\}_{k=1}^{\infty}$.

On the other hand, if $S \subset\{\ell \geq 0\}$ is compact and $z_{\infty}$ is any accumulation point of the sequence $\left\{z_{k}\right\}_{k=1}^{\infty} \subset Z(S)$ mentioned above, then taking the limit of

$$
\ell\left(x_{k}, z_{k}\right)=s_{k} \ell\left(x_{k}, y_{k}\right) \text { and } \quad \ell\left(z_{k}, y_{k}\right)=\left(1-s_{k}\right) \ell\left(x_{k}, y_{k}\right)
$$

along a subsequence $\left(x_{k(j)}, y_{k(j)}\right) \rightarrow\left(x_{\infty}, y_{\infty}\right)$ in $S$ with $z_{k(j)} \rightarrow z_{\infty}$ and $s_{k(j)} \rightarrow \bar{s}$, the continuity of $\ell$ from Lemma 2.3 shows $z_{\infty} \in Z_{\bar{s}}\left(x_{\infty}, y_{\infty}\right)$ to establish compactness of $Z(S)$. If $s_{k}=s$ for each $k$ then $\bar{s}=s$, so we have also established compactness of $Z_{s}(S)$.

Remark 2.6 Note $\ell(x, y)>0$ implies $Z_{0}(x, y)=\{x\}$ and $Z_{1}(x, y)=\{y\}$. Indeed, if e.g. $x \neq z \in Z_{0}(x, y)$, concatenating the action minimizing segment linking $x$ to $z$ with that linking $z$ to $y$ yields an action minimizing segment from $x$ to $y$ which changes causal type from null to timelike, contradicting the smoothness of geodesics which follows from the Euler-Lagrange equation they satisfy.

Lemma 2.3 asserts $\operatorname{sing}(\ell)$ to be closed. Define the timelike injectivity locus TIL $\subset$ TM to be the (unique) connected component of $\exp ^{-1}[M \times M \backslash$ $\sin g(\ell)]$ containing the zero section in its boundary. Let $T I L_{0}$ be the subset of the closure of TIL on which the exponential map remains well-defined, and $T I L_{+}:=T I L_{0} \cap \exp ^{-1}[\{\ell>0\}]$. Recall that a map between topological spaces is proper if the preimage of any compact set is compact. 
Corollary 2.7 (Proper action of the Lorentzian exponential) Global hyperbolicity of $(M, g)$ implies the Lorentzian exponential restricts to a proper map $\overline{\exp }: T I L_{+} \longrightarrow\{\ell>0\}$ on $T I L_{+} \subset T M$.

Proof. Let $\overline{\exp }$ denote the restriction of exp to TIL $L_{+}$. Given $S \subset\{\ell>$ 0\} $\subset M \times M$ compact and $\left(p_{i}, x_{i}\right) \in \overline{\exp }^{-1} S$, set $y_{i}=\exp _{x_{i}} p_{i}$ and $z_{i}:=$ $\exp _{x_{i}} \frac{1}{2} p_{i}$. The compactness of $S$ and $Z_{1 / 2}(S)$ shown in Lemma 2.5 provide a subsequence $\left(x_{i(k)}, y_{i(k)}, z_{i(k)}\right)$ converging to a limit $\left(\bar{x}, \bar{y}, z_{0}\right) \in S \times Z_{1 / 2}(S)$, with $z_{0}$ being the chronological midpoint of an action minimizing segment joining $\bar{x}$ to $\bar{y}$. Thus $\left(\bar{x}, z_{0}\right) \notin \operatorname{sing}(\ell)$, so $\overline{\exp }^{-1}$ acts diffeomorphically near $\left(\bar{x}, z_{0}\right)$. Since $z_{0}=\lim _{k \rightarrow \infty} \exp _{x_{i(k)}} \frac{1}{2} p_{i(k)}$ we conclude $p_{0}:=\lim _{k \rightarrow \infty} p_{i(k)}$ exists and deduce $z_{0}=\exp _{\bar{x}} \frac{1}{2} p_{0}$ and $\bar{y}=\exp _{\bar{x}} p_{0}$. Thus $\left(p_{0}, \bar{x}\right) \in \overline{\exp }^{-1} S$, to establish that $\overline{\exp }^{-1} S$ is compact and $\overline{\exp }$ is proper.

Lemma 2.8 (Selecting midpoints on the timelike cut locus) The maps $z_{s}$ from Lemma 2.4 can be measurably extended to $\{\ell>0\}$ by $\bar{z}_{s}$ so that $\ell(x, y)>0$ implies $s \in[0,1] \mapsto \bar{z}_{s}(x, y)$ is a proper-time maximizing geodesic segment joining $x$ to $y$.

Proof. Global hyperbolicity of $(M, g)$ implies the infimum (2) is attained [13] 84] hence $Z_{1 / 2}(S)$ is non-empty (16) for each $\emptyset \neq S \subset\{\ell>0\}$; it is closed if $S$ is, due to the continuity of $\ell(x, y)$ stated in Lemma 2.3, in particular $Z_{s}(x, y)$ is closed and non-empty for $(x, y) \in\{\ell>0\}$. The same lemma shows $\{\ell \leq 0\}$ to be closed. Thus $\exp ^{-1}[\{\ell>0\}]$ is open. Define the timelike injectivity locus, TIL $\subset T M$ to be the connected component of $\exp ^{-1}[\operatorname{sing}(\ell)]$ containing the zero section in its boundary. Set $T I L(x):=$ $\left\{v \in T_{x} M \mid(x, v) \in T I L\right\}$ and let $\overline{T I L}(x)$ denote its closure. Then $V(x, y):=$ $\overline{T I L}(x) \cap \exp _{x}^{-1} Z_{1 / 2}(x, y)$ is closed and non-empty for each $(x, y) \in\{\ell>0\}$, and $V(S):=\cup_{(x, y) \in S} V(x, y)$ is closed if $S \subset\{\ell>0\}$ is. This shows $V$ to be a measurable correspondence with non-empty closed values between the space $\{\ell>0\}$ metrized by $d_{\tilde{g}} \oplus d_{\tilde{g}}$ and the Polish space $T M$, in the terminology of Aliprantis and Border; it therefore admits a measurable selection $v:\{\ell>$ $0\} \longrightarrow M$ such that $v(x, y) \in V(x, y)$ according to the Kuratowski-RyllNardzewski Theorem, e.g. 18.13 of [4]. Now $\bar{z}_{s}(x, y):=\exp _{s} s \bar{v}(x, y)$ gives the desired measurable extension of $z_{s}$ from $M \times M \backslash \operatorname{sing}(\ell)$ to $\{\ell>0\}$. By construction, $\ell(x, y)>0$ implies that $s \in[0,1] \mapsto \bar{z}_{s}(x, y)$ is a proper-time maximizing geodesic segment joining $x$ to $y$.

We next identify the cases of equality in Eckstein and Miller's reverse triangle inequality [38] under the simplifying hypothesis $q \neq 1$ : 
Proposition 2.9 (Reverse triangle inequality and cases of equality) Fix $0<q<1$. If $\mu_{1}, \mu_{2}, \mu_{3} \in \mathcal{P}(M)$ and $\ell_{q}\left(\mu_{1}, \mu_{2}\right) \neq-\infty \neq \ell_{q}\left(\mu_{2}, \mu_{3}\right)$ then

$$
\ell_{q}\left(\mu_{1}, \mu_{3}\right) \geq \ell_{q}\left(\mu_{1}, \mu_{2}\right)+\ell_{q}\left(\mu_{2}, \mu_{3}\right) ;
$$

moreover, if $\mu_{1}\left[X_{1}\right]=1=\mu_{3}\left[X_{3}\right]$ and $\ell_{q}\left(\mu_{1}, \mu_{2}\right)+\ell_{q}\left(\mu_{2}, \mu_{3}\right)<\infty$ then the inequality is strict unless $\mu_{2}\left[Z\left(X_{1} \times X_{3}\right)\right]=1$.

Conversely, if (i) $\ell_{q}\left(\mu_{1}, \mu_{3}\right) \in(0, \infty)$, (ii) equality holds in (18), and (iii) both suprema (6) defining $\ell_{q}\left(\mu_{1}, \mu_{2}\right)$ and $\ell_{q}\left(\mu_{2}, \mu_{3}\right)$ are attained, then there exists $\omega \in \mathcal{P}\left(M^{3}\right)$ for which $\pi_{i j}:=\operatorname{proj}_{i j \#} \omega \in \Pi\left(\mu_{i}, \mu_{j}\right)$ is $\ell^{q}$-optimal for each $i<j$ with $i, j \in\{1,2,3\}$ and each $(x, y, z) \in \operatorname{spt} \omega$ satisfies

$$
\ell(x, y)=s \ell(x, z) \text { and } \ell(y, z)=(1-s) \ell(x, z)
$$

with $s:=\ell_{q}\left(\mu_{1}, \mu_{2}\right) / \ell_{q}\left(\mu_{1}, \mu_{3}\right)$ and $\operatorname{proj}_{i j}\left(x_{1}, x_{2}, x_{3}\right)=\left(x_{i}, x_{j}\right)$. If, in addition, $\pi_{13}[S]=1$ for some $S \subset M \times M$ then $\mu_{2}$ vanishes outside $Z_{s}(S)$. In particular, if $Z_{s}(x, y)=\left\{z_{s}(x, y)\right\}$ holds for $\pi_{13}$-a.e. $(x, y)$, then $\omega=$ $\left(z_{0} \times z_{s} \times z_{1}\right)_{\#} \pi_{13}$ and $\mu_{2}=z_{s \#} \pi_{13}$ in the notation of Definition 2.2.

Proof. If either term on the right hand side of (18) diverges to $-\infty$ there is nothing to prove. Otherwise, given $\epsilon>0$ there exist $\pi_{12} \in \Pi_{\beth}\left(\mu_{1}, \mu_{2}\right)$ and $\pi_{23} \in \Pi_{\preceq}\left(\mu_{2}, \mu_{3}\right)$ which are nearly $\ell^{q}$-optimal, in the sense that

$$
\ell^{q}\left[\pi_{i j}\right]:=\int_{M^{2}} \ell^{q} d \pi_{i j} \geq \min \left\{\ell_{q}\left(\mu_{i}, \mu_{j}\right)-\epsilon, \epsilon^{-1}\right\}
$$

for $j=i+1 \in\{2,3\}$. Disintegrate $d \pi_{12}(x, y)=d \mu_{2}(y) d \pi_{12}^{y}(x)$ and $d \pi_{23}(y, z)=$ $d \mu_{2}(y) d \pi_{23}^{y}(z)$ and define $\omega$ by 'gluing': i.e.,

$$
\int_{M^{3}} \phi(x, y, z) d \omega(x, y, z)=\int_{M} d \mu_{2}(y) \int_{M^{2}} \phi(x, y, z) d \pi_{12}^{y}(x) d \pi_{23}^{y}(z),
$$

as in e.g. [92, Definition 16.1]. Then $\pi_{13}:=\operatorname{proj}_{13 \#} \omega \in \Pi\left(\mu_{1}, \mu_{3}\right)$ and

$$
\begin{aligned}
\ell_{q}\left(\mu_{1}, \mu_{3}\right) & \geq\|\ell(x, z)\|_{L^{q}\left(d \pi_{13}\right)} \\
& =\|\ell(x, z)\|_{L^{q}(d \omega)} \\
& \geq\|\ell(x, y)+\ell(y, z)\|_{L^{q}(d \omega)} \\
& \geq\|\ell(x, y)\|_{L^{q}(d \omega)}+\|\ell(y, z)\|_{L^{q}(d \omega)} \\
& \geq \min \left\{\ell_{q}\left(\mu_{1}, \mu_{2}\right)+\ell_{q}\left(\mu_{2}, \mu_{3}\right)-2 \epsilon, \epsilon^{-1}-\epsilon\right\}
\end{aligned}
$$


where the inequalities follow from the definition (6) of $\ell_{q}$, reverse triangle inequality (44) for Lorentz distance, and the (reverse) Minkowski inequality for $q \in(0,1]$. In particular $\pi_{13} \in \Pi_{\preceq}\left(\mu_{1}, \mu_{3}\right)$. Since $\epsilon>0$ was arbitrary, (18) is established. Assume $\mu_{i}\left[X_{i}\right]=1$, so $\omega$ vanishes outside $X_{1} \times M \times X_{3}$. For $(x, y, z) \in X_{1} \times M \times X_{3}$ with $\ell(x, z) \geq 0$, inequality (4) holds strictly unless $y \in Z\left(X_{1} \times X_{3}\right)$; since $\ell(x, z) \geq 0$ holds $\omega$-a.e., $\ell_{q}\left(\mu_{1}, \mu_{2}\right)+\ell_{q}\left(\mu_{2}, \mu_{3}\right)<\infty$ implies (18) is strict unless $\omega$ vanishes outside $X_{1} \times Z\left(X_{1} \times X_{3}\right) \times X_{3}$, or equivalently, unless $\mu_{2}=\operatorname{proj}_{2 \#} \omega$ vanishes outside $Z\left(X_{1} \times X_{3}\right)$.

Now assume the suprema (6) defining $\ell_{q}\left(\mu_{1}, \mu_{2}\right)$ and $\ell_{q}\left(\mu_{2}, \mu_{3}\right)$ are both finite and attained, so that we can henceforth fix $\epsilon=0$ in the argument above. When (18) is saturated, each of the three inequalities in the preceding chain of claims must be saturated as well. Saturation of the first asserts $\ell^{q}$-optimality of $\pi_{13}$. For $\omega$-a.e. $(x, y, z)$, saturation of the second shows $\ell(x, z)=\ell(x, y)+\ell(y, z)$, while the third (Minkowski) asserts the existence of $s \in[0,1]$ such that that $(1-s) \ell(x, y)=s \ell(y, z)$. Combining the last two identities asserts that (19) holds $\omega$-a.e.; comparison with (18) forces $s:=\ell_{q}\left(\mu_{1}, \mu_{2}\right) / \ell_{q}\left(\mu_{1}, \mu_{3}\right)$. Since $\omega$ vanishes outside the closed set $\{(x, y, z) \mid$ $\min \{\ell(x, y), \ell(x, z), \ell(y, z)\} \geq 0\}$, the continuity of $\ell$ from Lemma 2.3 implies (19) extends to all $(x, y, z) \in \operatorname{spt} \omega$.

Now suppose $\pi_{13}[S]=1$ for some $S \subset M \times M$. Then $\omega[\tilde{S}]=1$ where $\tilde{S}:=\{(x, y, z) \mid(x, z) \in S\}$. For any Borel set $A \subset M$ disjoint from $Z_{s}(S)$, it follows that $M \times A \times M$ is disjoint from $\tilde{S}$, hence $\mu_{2}(A)=\omega[M \times A \times M]=0$ as desired. For example, suppose $Z_{s}(x, y)=\left\{z_{s}(x, y)\right\}$ holds for $\pi_{13}$-a.e. $(x, y)$. Then $\omega$ vanishes outside the graph of $z_{0} \times z_{s} \times z_{1}:$ spt $\pi_{13} \longrightarrow M \times M \times M$, whence $\omega=\left(z_{0} \times z_{s} \times z_{1}\right)_{\#} \pi_{13}$ by e.g. Lemma 3.1 of [2].

Corollary 2.10 (Interpolants inherit compact support) Let $\left(\mu_{s}\right)_{s \in[0,1]} \subset$ $\mathcal{P}(M)$ be a q-geodesic on globally hyperbolic spacetime $(M, g)$. If $\mu_{0}$ and $\mu_{1}$ have compact support, then $\mathrm{spt} \mu_{s} \subset Z_{s}\left(\operatorname{spt}\left[\mu_{0} \times \mu_{1}\right]\right)$ and the latter is compact for each $s \in[0,1]$.

Proof. Fix a $q$-geodesic $\left(\nu_{t}\right)_{t \in[0,1]} \subset \mathcal{P}(M)$ with compactly supported endpoints. Setting $X_{t}:=\operatorname{spt} \nu_{t}$, Lemma 2.5 shows the compactness of $Z:=$ $Z\left(X_{0} \times X_{1}\right)$ and $Z_{s}:=Z_{s}\left(X_{0} \times X_{1}\right)$. Let $0 \leq s<t \leq 1$ be arbitrary. From definition (8) we see $0<\ell_{q}\left(\nu_{s}, \nu_{t}\right)<\infty$, so taking $\left(\mu_{1}, \mu_{2}, \mu_{3}\right)=\left(\nu_{0}, \nu_{s}, \nu_{1}\right)$ yields equality in (18). The first part of Proposition 2.9 asserts $\nu_{s}$ vanishes outside of $Z$ - hence is compactly supported. Since $\sup _{Z} \ell<\infty$, the suprema (6) defining $\ell_{q}\left(\nu_{s}, \nu_{t}\right)$ is attained, and the second part of Proposition 2.9 asserts spt $\nu_{s} \subset Z_{s}$ as desired. 
Theorem 2.11 (Existence of $q$-geodesics) Let $(M, g)$ be a globally hyperbolic spacetime and $0<q \leq 1$. Fix $\mu, \nu \in \mathcal{P}(M)$ and suppose (6) is finite and attained by some $\pi \in \Pi(\mu, \nu)$ with $\ell>0$ holding $\pi$-a.e. Then (i) $\mu_{s}:=\bar{z}_{s \#} \pi$ defines a q-geodesic $s \in[0,1] \mapsto \mu_{s} \in \mathcal{P}(M)$ where $\bar{z}_{s}(x, y)$ is from Lemma 2.8. (ii) If $0 \leq s<t \leq 1$ then $\left(\bar{z}_{s} \times \bar{z}_{t}\right)_{\#} \pi$ is $\ell^{q}$-optimal. (iii) If $\mu_{0}$ and $\mu_{1}$ are compactly supported and the maximum (6) is uniquely attained and $\pi[\operatorname{sing}(\ell)]=0$, then the q-geodesic joining $\mu=\mu_{0}$ to $\nu=\mu_{1}$ is unique.

Proof. (i)-(ii) Taking $\mu, \nu \in \mathcal{P}(M)$ and $0 \leq s<t \leq 1$ as hypothesized, suppose (6) is finite and attained by some $\pi \in \Pi_{\preceq}(\mu, \nu)$ with $\ell>0$ holding $\pi$-a.e. Use the extension $\bar{z}_{s}$ of $z_{s}$ from Lemma 2.8 to define $\mu_{s}:=\bar{z}_{s \#} \pi$. Trying $\left(\bar{z}_{s} \times \bar{z}_{t}\right)_{\#} \pi \in \Pi_{\preceq}\left(\mu_{s}, \mu_{t}\right)$ in (마) shows

$$
\begin{aligned}
\ell_{q}\left(\mu_{s}, \mu_{t}\right)^{q} & \geq \int \ell\left(\bar{z}_{s}(x, y), \bar{z}_{t}(x, y)\right)^{q} d \pi(x, y) \\
& =(t-s)^{q} \int \ell(x, y)^{q} d \pi(x, y) \\
& =(t-s)^{q} \ell_{q}\left(\mu_{0}, \mu_{1}\right)^{q}
\end{aligned}
$$

from the fact that $s \in[0,1] \mapsto \bar{z}(x, y)$ is a proper time maximizing segment for $\pi$-a.e. $(x, y)$ and the optimality of $\pi \in \Pi_{\preceq}\left(\mu_{0}, \mu_{1}\right)$.

These lower bounds are finite and positive by hypothesis, and imply

$$
\ell_{q}\left(\mu_{0}, \mu_{s}\right)+\ell_{q}\left(\mu_{s}, \mu_{t}\right)+\ell_{q}\left(\mu_{t}, \mu_{0}\right) \geq \ell_{q}\left(\mu_{0}, \mu_{1}\right) .
$$

The reverse triangle inequality proved in Proposition 2.9 forces both inequalities to become equalities. Thus $s \in[0,1] \mapsto \mu_{s} \in \mathcal{P}(M)$ is a $q$-geodesic, and $\left(\bar{z}_{s} \times \bar{z}_{t}\right)_{\#} \pi$ is $\ell^{q}$-optimal.

(iii) If $\mu_{0}$ and $\mu_{1}$ are compactly supported, Corollary 2.10 asserts the same is true for $\mu_{s}$. When the maximum (6) is uniquely attained by $\pi \in \Pi_{\preceq}(\mu, \nu)$, uniqueness of $\mu_{s}$ follows from the last assertion in Proposition [2.9], whose hypotheses are satisfied because $\pi_{13}=\pi$ was assumed to vanish on $\operatorname{sing}(\ell)$, and because compact support guarantees the suprema (6) defining $\ell\left(\mu_{s}, \mu_{t}\right)$ in (8) is attained for each $0 \leq s<t \leq 1$.

\section{Lagrangian, Hamiltonian, Lorentz distance}

In this section, first- and second-variation formulas are used to establish properties of the Lorentz distance which will be useful throughout, along 
with convex-analytic properties of the Lagrangian and Hamiltonian which define it. Although it would not be surprising to learn they have been studied elsewhere, we have not seen the family of Lagrangians

$L(v ; q):=\left\{\begin{array}{cl}-\left(g_{a b}(x) v^{a} v^{b}\right)^{q / 2} / q & \text { if } v \text { is future-directed and } g_{a b}(x) v^{a} v^{b} \geq 0 \\ +\infty & \text { else }\end{array}\right.$

discussed previously — apart from the case $q=1$ [88. Propositions 3.4 and Theorem 3.5 are inspired by corresponding results from the Riemannian setting [36], but the Lorentzian versions appear to be new. They are based on concepts from non-smooth analysis recalled here which will also be useful later: sub- and superdifferentiability, semiconvexity and -concavity, approximate derivatives.

On a Riemannian manifold $\left(M^{n}, \tilde{g}\right)$, a function $u: M \longrightarrow[-\infty,+\infty]$ is said to be subdifferentiable at $x$ with subgradient $p \in T_{x}^{*} M$ if $x \in \operatorname{Dom} u:=$ $u^{-1}(\mathbf{R})$ and

$$
u\left(\exp _{x} v\right) \leq u(x)+p[v]+o\left(|v|_{\tilde{g}}\right)
$$

holds for small $v \in T_{x} M$. Here $p[v]$ denotes the duality pairing of $p$ with $v$. It doesn't matter whether the Riemannian or Lorentzian exponential is used in this definition, since they agree to order $o\left(|v|_{\tilde{g}}\right)$. The set of subgradients for $u$ at $x$ is denoted by $\partial u(x)$, or by $\partial . u(x)$ when we need to distinguish it from the set $\partial u(x)$ of supergradients. Here $p$ is a supergradient if inequality (21) is reversed, in which case we say $u$ is superdifferentiable at $x$. If $u$ has both a sub- and a supergradient at $x$, then $u$ is differentiable at $x$, in which case the super- and subdifferentials $\partial \cdot u(x)=\partial u(x)=\{D u(x)\}$ agree, and we write $x \in \operatorname{Dom} D u$.

Lemma 3.1 (Convex Lagrangian and Hamiltonian) Fix $0<q<1$ and a point $x$ on a Lorentzian manifold $\left(M^{n}, g\right)$. (i) The Lagrangian (20) is convex on $T_{x} M$; where $L<0$ it is smooth and its Hessian

$$
|v|^{2-q} g^{i j} g^{k l} \frac{\partial^{2} L}{\partial v^{k} \partial v^{l}}=(2-q)|v|^{-2} v^{i} v^{j}-g^{i j}
$$

is positive-definite, so strict convexity fails only on the future light cone. (ii) Subdifferentiability of $L(\cdot ; q)$ fails throughout the light cone. (iii) The corresponding Hamiltonian on the cotangent space $T_{x}^{*} M$ is given by

$H(p ; q):=\left\{\begin{array}{cl}-\left(g^{a b}(x) p_{a} p_{b}\right)^{q^{\prime} / 2} / q^{\prime} & \text { if } p \text { is past-directed and } g^{a b}(x) p_{a} p_{b}>0 \\ +\infty & \text { else }\end{array}\right.$ 
with $\frac{1}{q}+\frac{1}{q^{\prime}}=1$; it satisfies $v=D H(D L(v ; q) ; q)$ and $p=D L(D H(p ; q) ; q)$ for all timelike future-directed $v \in T_{x} M$ and timelike past-directed $p \in T_{x}^{*} M$.

Proof. (i) In the interior of the future cone, $L$ is smooth and we compute that $\frac{\partial L}{\partial v^{i}}=-|v|^{q-2} g_{i j} v^{j}$ is past-directed (because of the minus sign) and thence (22) with $|v|:=\sqrt{v^{a} g_{a b} v^{b}}$. Since $2-q>1$ and the reverse CauchySchwartz inequality asserts $\left(w^{a} g_{a b} v^{b}\right)^{2} \geq\left(w^{q} g_{a b} w^{b}\right)\left(v^{a} g_{a b} v^{b}\right)$ whenever $v$ is timelike, we conclude $D^{2} L$ is non-negative definite, c.f. $\S 2.4$ of [19]; the obsevation that $w \neq 0$ is spacelike whenever it is orthogonal to $v$ improves this to positive-definiteness. Since the future cone $L \geq 0$ is convex and $L$ is continuous on it and $+\infty$ outside, it follows that $L$ is convex on $T_{x} M$.

(ii) For $q<1$ we see $|D L(v ; q)|=|v|^{q-1}$ diverges as $|v| \rightarrow 0$. This shows the subdifferential $\partial L(v ; q)$ is empty at each point $v$ on the light cone, since [81, Theorem 25.6] asserts $\partial L(v ; q)=N+A$ where $A$ is the set of accumulation points of $D L\left(v_{k} ; q\right)$ with $v_{k} \rightarrow v$, and $N$ is the normal ray to the light cone at $v$. In this case $A$ is empty.

(iii) We readily see that $D H$ and $D L$ invert each other on the specified cones using $\frac{\partial H}{\partial p_{k}}=-|p|^{q^{\prime}-2} g^{k j} p_{j}$ and $(q-1)\left(q^{\prime}-1\right)=1$.

Corollary 3.2 (The classical Lagrangian and Hamiltonian) The limit $L(v ; 1)=\lim _{q \rightarrow 1^{-}} L(v ; q)$ inherits convexity from $L(v ; q)$, but fails to be strictly convex along any ray through the origin. (ii) Its convex dual Hamilton $H(p ; 1):=\sup _{v \in T_{x} M} p[v]-L(v ; q)$ is the indicator function of a past-directed solid hyperboloid:

$$
H(p ; 1)=\left\{\begin{array}{cl}
0 & \text { if } p \text { is past-directed and } g^{a b}(x) p_{a} p_{b} \geq 1 \\
+\infty & \text { else. }
\end{array}\right.
$$

(iii) Although $L$ is smooth in the interior of the future cone, subdifferentiability of $L(v ; 1)$ fails at each point on the lightcone apart from the origin, where its subdifferential $\partial L(\mathbf{0} ; 1)=\{p \mid H(p ; 1)=0\}$ is the solid hyperboloid.

Proof. (i) Lemma 3.1 makes convexity of $L(v ; q)$ and hence its $q \rightarrow 1^{-}$ limit $L(v ; 1)$ clear. Strict convexity fails along each ray due to the positive 1-homogeneity of $L(\lambda v ; 1)=\lambda L(v ; 1)$ for each $\lambda>0$.

(ii) The Legendre transform of the limit is the limit of the Legendre transforms:

$$
H(p ; 1)=\lim _{q \rightarrow 1^{-}} H(p ; q)
$$


Formula (24) now follows from (23).

(iii) Since $p \in \partial L(v ; 1)$ if and only if $v \in \partial H(p ; 1)$, we deduce $\{p \mid$ $H(p, 1)=0\} \subset \partial L(\mathbf{0} ; 1)$. Equality must hold since $H(\cdot ; 1)$ is not subdifferentiable outside its zero set. Each slope $p$ in the interior of the hyperboloid Dom $H(\cdot ; 1)$ supports the graph of $L(\cdot ; 1)$ only at the origin. Each slope on the hyperboloid boundary supports the graph of $L(\cdot ; 1)$ on an entire ray. Apart from the origin, this ray lies in the interior of the future-cone Dom $L(\cdot ; 1)$, since the hyperboloid is strictly convex. Thus $L(\cdot ; 1)$ cannot be subdifferentiable on the lightcone, except at the origin.

Recall the following definition of semiconvexity from, e.g. [17] [67], which is independent of the choice of Riemannian metric $\tilde{g}$ on $M$.

Definition 3.3 (Semiconvexity) Fix $U \subset M$ open. A function $u: U \longrightarrow$ $\mathbf{R}$ is semiconvex on $U$ if there is a constant $C \in \mathbf{R}$ such that

$$
\liminf _{w \rightarrow 0} \frac{u\left(\exp _{x}^{\tilde{g}} w\right)+u\left(\exp _{x}^{\tilde{g}}-w\right)-2 u(x)}{2|w|_{\tilde{g}}^{2}} \geq C .
$$

for all $x \in U$. The largest such $C$ is called the the semiconvexity constant of $u$ on $U$. Similarly, $u$ is called semiconcave if $-u$ is semiconvex.

Proposition 3.4 (Semiconvexity of Lorentz distance) For any smooth Riemannian metric $\tilde{g}$ on a globally hyperbolic manifold $(M, g)$, the limit

$$
\tilde{C}(x, y):=\liminf _{w \rightarrow 0} \frac{\ell\left(\exp _{x}^{\tilde{g}} w, y\right)+\ell\left(\exp _{x}^{\tilde{g}}-w, x\right)-2 \ell(x, y)}{2|w|_{\tilde{g}}^{2}}
$$

is continuous and real-valued on $\{(x, y) \mid \ell(x, y)>0\}$.

Proof. Suppose $\ell_{0}:=\ell(x, y)>0$ and let $\sigma$ be an action minimizing geodesic from $x=\sigma(0)$ to $y=\sigma(1)$ with $|\dot{\sigma}(s)|_{g}=\ell_{0}$. Given $w \in T_{x} M$ with $|w|_{\tilde{g}}=1$, let $w(s)$ be the Lorentzian parallel transport of $w$ along $\sigma$ and set

$$
W(s):=(1-s) w(s)
$$

so $W^{\prime}(s)=-w(s)$. Use the Riemannian exponential map to define a variation $\beta(r, s)$ around $\sigma(s)$ by

$$
\sigma_{r}(s):=\beta(r, s)=\exp _{\sigma(s)}^{\tilde{g}}(r W(s))
$$


with variable initial point $\sigma_{r}(0)=\exp _{x}^{\tilde{g}} r w$ but fixed final point $\sigma_{r}(1)=y$. Now use (2) to estimate

$$
\ell\left(\exp _{x}^{\tilde{g}} r w, x\right) \geq-a(r),
$$

by the action $a(r):=A\left[\sigma_{r} ; 1\right]$; equality holds when $r=0$. Thus we can bound the Riemannian Hessian of the Lorentz distance by that of the length (or action) functional $-A[\sigma ; 1]$ :

$$
\frac{\ell\left(\exp _{x}^{\tilde{g}} r w, y\right)+\ell\left(\exp _{x}^{\tilde{g}}-r w, y\right)-2 \ell(x, y)}{2 r^{2}} \geq-\frac{a(r)+a(-r)-2 a(0)}{2 r^{2}}
$$

The expression on the right converges: letting $\frac{D}{d r}$ denote Lorentzian covariant differentiation along the curve $r \mapsto \sigma_{r}(s)$, its limit is given by Synge's second variation formula, e.g. Theorem 10.4 of [77]:

$$
\left.\ell_{0} \frac{d^{2} a}{d r^{2}}\right|_{r=0}=-\left.\left\langle\sigma^{\prime}, \frac{D}{\partial r} \frac{\partial \beta}{\partial r}\right\rangle\right|_{0} ^{1}-\int_{0}^{1}\left[\left\langle W_{\perp}^{\prime}, W_{\perp}^{\prime}\right\rangle-R\left(W_{\perp}, \sigma^{\prime}, W_{\perp}, \sigma^{\prime}\right)\right] d s,
$$

where $W_{\perp}:=W-\left\langle W, \sigma^{\prime}\right\rangle \sigma^{\prime} / \ell_{0}^{2}=(1-s) w_{\perp}(s)$ is the projection of $W$ onto the orthogonal subspace of the geodesic $\sigma$, whose geodesy implies $W_{\perp}^{\prime}=$ $W^{\prime}-\left\langle W^{\prime}, \sigma^{\prime}\right\rangle \sigma^{\prime} / \ell_{0}^{2}=-w_{\perp}(s)$. Thus

$$
\begin{aligned}
\left.\ell_{0} \frac{d^{2} a}{d r^{2}}\right|_{r=0} & =-\left.\left\langle\sigma^{\prime}, \frac{D}{\partial r} \frac{\partial \beta}{\partial r}\right\rangle\right|_{0} ^{1}+\int_{0}^{1}\left[(1-s)^{2} R\left(w_{\perp}, \sigma^{\prime}, w_{\perp}, \sigma^{\prime}\right)-\left|w_{\perp}\right|_{g}^{2}\right] d s \\
& \leq-\left.\left\langle\sigma^{\prime}, \frac{D}{\partial r} \frac{\partial \beta}{\partial r}\right\rangle\right|_{0} ^{1}+\sup _{s \in[0,1]}(1-s)^{2} R\left(w_{\perp}, \sigma^{\prime},{ }_{\perp}, \sigma^{\prime}\right)-\left\langle w_{\perp}, w_{\perp}\right\rangle_{g} \\
& =: C\left(x, y ; w(0), \sigma^{\prime}(0)\right),
\end{aligned}
$$

where $C\left(x, y ; w, \sigma^{\prime}\right)$ is a continuous real-valued function of all four of its arguments (since the expression under the supremum is smooth - hence locally uniformly continuous - in the same variables, and $[0,1]$ is compact). Similarly, continuity of

$$
C(x, y):=\sup _{|w|_{\left(T_{x} M, \tilde{g}\right)}=1} \sup _{v \in \overline{\exp }_{x}^{-1} y} C(x, y ; w, v)
$$

on $\{\ell>0\}$ follows from the compactness of $\overline{\exp }_{x}^{-1} y$ established in Corollary 2.7. Taking $\tilde{C}(x, y)=-C(x, y) / \ell(x, y)$, the continuity of $\ell(x, y)$ recalled in Lemma 2.3 concludes the proof of (25). 
Theorem 3.5 (Semiconcavity fails on the timelike cut locus) If $(x, y) \in$ $\{\ell>0\} \cap \operatorname{sing}(\ell)$ then

$$
\sup _{0<|w|_{\tilde{g}}<1} \frac{\ell\left(\exp _{x}^{\tilde{g}} w, y\right)+\ell\left(\exp _{x}^{\tilde{g}}-w, y\right)-2 \ell(x, y)}{2|w|_{\tilde{g}}^{2}}=+\infty
$$

Proof. Fix $(x, y) \in\{\ell>0\} \cap \operatorname{sing}(\ell)$. If $x$ is a cut point - meaning multiple action minimizing curves link $x$ to $y$ - the proof is easy. Therefore, assume $x$ is a conjugate point of $y$. To derive a contradiction, assume also

$$
\limsup _{w \rightarrow 0} \frac{\ell\left(\exp _{x}^{\tilde{g}} w, y\right)+\ell\left(\exp _{x}^{\tilde{g}}-w, y\right)-2 \ell(x, y)}{2|w|_{\tilde{g}}^{2}}<\tilde{C} \in \mathbf{R} .
$$

This means the function $u(\cdot):=\ell(\cdot, y)$ has a quadratic upper bound at $x$. Proposition 3.4 implies $u(\cdot)$ also admits a quadratic lower bound at the same point. To first order, these bounds must agree, hence $u(\cdot)$ is differentiable at $x$. We claim (27) implies an analogous bound (28) for the second difference quotients of $u$ along Lorentzian rather than Riemannian geodesics, but possibly with a larger constant $C>\tilde{C}$. Indeed, given a Riemannian unit vector $w \in T_{x} M$, letting $\gamma_{r}=\exp _{x} r w$, in Riemannian normal coordinates around $x$ we find

$$
u\left(\gamma_{r}\right)+u\left(\gamma_{-r}\right)-2 u(x) \leq \frac{\partial u}{\partial x^{\alpha}}\left[\gamma_{r}+\gamma_{-r}-2 x\right]^{\alpha}+\tilde{C} \sum_{\alpha=1}^{n}\left(\gamma_{r}^{\alpha}\right)^{2}+\left(\gamma_{-r}^{\alpha}\right)^{2}
$$

for $r$ sufficiently small. Thus

$$
\limsup _{w \rightarrow 0} \frac{\ell\left(\exp _{x} w, y\right)+\ell\left(\exp _{x}-w, y\right)-2 \ell(x, y)}{2|w|_{\tilde{g}}^{2}} \leq C
$$

where

$$
C=\tilde{C}+|D u|_{\tilde{g}} \sup _{|w| \tilde{g}=1}\left|\frac{\tilde{D}}{\partial r} \frac{d \gamma_{r}}{d r}\right|_{\tilde{g}}<\infty
$$

and $\frac{\tilde{D}}{\partial r}$ denotes Riemannian covariant differentiation.

Let $\sigma(s)$ be the proper time maximizing geodesic segment joining $x=\sigma(0)$ to $y=\sigma(1)$, and define its index form by

$$
I\left(W_{1}, W_{2}\right):=-\int_{0}^{1}\left\langle W_{1 \perp}^{\prime}, W_{2 \perp}^{\prime}\right\rangle-R\left(W_{1 \perp}, \sigma^{\prime}, W_{2 \perp}, \sigma^{\prime}\right) d s .
$$


where $W_{i}^{\prime}$ denotes the covariant derivative of $W_{i}$ along $\sigma$, and $W_{\perp}:=W-$ $g\left(W, \sigma^{\prime}(s)\right) \sigma^{\prime}(s) /\left|\sigma^{\prime}(s)\right|_{g}^{2}$ denotes the component of $W$ orthogonal to $\sigma$.

Let $U(s)$ be a non-zero Jacobi field along $\sigma$ vanishing at its endpoints $s \in\{0,1\}$. Notice $w:=U^{\prime}(0)$ cannot be a multiple of $\sigma^{\prime}(0)$, since the initial conditions $\left(0, \sigma^{\prime}(0)\right)$ generate the solution $(s \sigma(s))_{s \in[0,1]}$ to Jacobi's equation which corresponds to simply stretching the geodesic. Thus the component $w_{\perp}$ of $w$ orthogonal to $\sigma^{\prime}(0)$ is spacelike. Scaling the Jacobi field $U$ and the Riemannian and Lorentzian metrics independently we may assume $\ell(x, y)=$ 1 and $|w|_{\tilde{g}}=1=-g\left(w_{\perp}, w_{\perp}\right)$. Let $w(s)$ be a parallel field along $\sigma$ with $w(0)=w$ and set $W(s):=(1-s) w(s)$. Fix $\epsilon>0$ small enough that

$$
I(W, W)<-C+\frac{2}{\epsilon}
$$

and then let $U_{\epsilon}(s):=U(s)+\epsilon W(s)$ be a perturbation of the Jacobi field in question. Introduce the variation $\sigma_{r}(s)=\beta(r, s):=\exp _{\sigma(s)} r U_{\epsilon}(s)$ around the geodesic segment $\sigma_{0}$. Its action is $a(r):=A\left[\sigma_{r} ; 1\right]$. Since $\sigma_{r}$ joins $\exp _{x} r \epsilon w$ to $y$, (2) implies

$$
\ell\left(\exp _{x} \epsilon r w, y\right) \geq-a(r),
$$

with equality when $r=0$. Thus, by assumption (28)

$$
\lim _{r \rightarrow 0} \frac{a(r)+a(-r)-2 a(0)}{r^{2} \epsilon^{2}} \geq-C .
$$

Noting that $r \mapsto \beta(r, s)$ is a geodesic for each $s \in[0,1]$, the endpoint terms vanish in Synge's second variation formula (26), giving

$$
\begin{aligned}
-\epsilon^{2} C & \leq a^{\prime \prime}(0) \\
& =I\left(U_{\epsilon}, U_{\epsilon}\right)+\left.g\left(\frac{D}{D r} \frac{\partial \beta}{\partial r}(r, s), \dot{\sigma}(s)\right)\right|_{(r, s)=(0,0)} ^{(r, s)=(0,1)} \\
& =I(U, U)+2 \epsilon I(U, W)+\epsilon^{2} I(W, W) \\
& <0+2 \epsilon g\left(w_{\perp}, w_{\perp}\right)-C \epsilon^{2}+2 \epsilon
\end{aligned}
$$

by our choice of $\epsilon$, since $U(s)$ Jacobi with vanishing endpoints implies $I(U, U)=$ 0 and $I(U, W)=-\left.g\left(W_{\perp}(s), U_{\perp}^{\prime}(s)\right)\right|_{s=0} ^{s=1}=g\left(w_{\perp}, w_{\perp}\right)=-1$, noting our choice of $W(s)$. This yields the contradiction desired to establish the theorem.

For convenient reference, we collect together several consequences of the foregoing analysis along with the known results of Lemma 2.3, and provide the deferred proof of $(\mathrm{c})$. 
Theorem 3.6 (Smoothness of Lorentz distance) Let $\left(M^{n}, g\right)$ be a globally hyperbolic spacetime. The Lorentz distance $\ell: M^{2} \longrightarrow[0, \infty) \cup\{-\infty\}$ defined by $q=1$ in (2) is (a) upper semicontinuous. It is (b) continuous on $\ell^{-1}([0, \infty))$, (c) smooth precisely on the complement of the closed set $\operatorname{sing}(\ell),(d)$ locally Lipschitz and locally semiconvex on the open set $\{\ell>0\}$. Moreover, if $y=\exp _{x} v$ and $x=\exp _{y} w$ for $(x, y) \in \ell^{-1}((0, \infty))$, then $-\frac{v_{*}}{\left|v_{*}\right|_{g}} \in \partial . u(x)$ and $-\frac{w_{*}}{\left|w_{*}\right|_{g}} \in \partial . \bar{u}(y)$, where $u(\cdot):=\ell(\cdot, y), \bar{u}(\cdot):=\ell(x, \cdot)$ and $v_{*}[\cdot]=g(v, \cdot)$. However, (e) the superdifferential of $\ell(\cdot, y)$ is empty at $x$ if $\ell(x, y)=0$ unless $x=y$, in which case the supergradients lie in the solid hyperboloid $\left\{p \in T_{x}^{*} M \mid H(p ; 1)=0\right\}$.

Proof. (a)-(b) were proven in Lemma 2.3, based on the continuity of the function $\ell_{+}:=\max \{\ell, 0\}$ from [19, Corollary 4.7] [77, Lemma 14.21-22].

(d) Openness of $\{\ell>0\}$ also follows from the continuity $\ell_{+}:=\max \{\ell, 0\}$. Semiconvexity of $\ell$ was established in Proposition 3.4 and, in combination with (b), implies $\ell$ is locally Lipschitz (since locally bounded convex functions are locally Lipschitz). Apart from an overall change of sign, the explicit form of the subgradient of $\ell(\cdot, y)$ at $x$ and $\ell(x, \cdot)$ at $y$ follows from the endpoint terms in the first variation formula, as in the proof of Proposition 10.15(i) of Villani 92. Although the statement of that Proposition requires $L \in C^{1}$, the proof makes it clear that it is enough to have tangent bundle estimates for $L$ and its first derivative in a neighbourhood of the minimizing geodesic joining $x$ to $y$. We have these estimates since $\ell(x, y)>0$ ensures the geodesic in question is timelike. As in the Riemannian case [67], an alternative proof may also be constructed based on the existence of convex neighbourhoods, the Gauss Lemma, and the reverse triangle inequality, similarly in strategy to the proof of (e) below.

(c) To see $\operatorname{sing}(\ell)$ is closed, suppose $\left(x_{k}, y_{k}\right) \in \operatorname{sing}(\ell)$ converge to $\left(x_{0}, y_{0}\right)$. By global hyperbolicity there is a proper-time maximizing segment joining $\left(x_{0}, y_{0}\right)$ [19, Theorem 3.18]; the only question is whether it has a proper-time maximizing extension in one and hence both [ibid, Theorem 9.12] directions. If $\ell\left(x_{0}, y_{0}\right) \leq 0$ then $\left(x_{0}, y_{0}\right) \in \operatorname{sing}(\ell)$, so assume $\ell\left(x_{0}, y_{0}\right)>0$. Then (a) shows $\ell\left(x_{k}, y_{k}\right)>0$ eventually. If $\left(x_{k}, y_{k}\right)$ are conjugate along a subsequence then $\left(x_{0}, y_{0}\right)$ are conjugate, hence in $\operatorname{sing}(\ell)$ [ibid, Theorem 9.11]. Otherwise, eventually each $\left(x_{k}, y_{k}\right)$ are joined by a pair of distinct proper-time maximizing segments. From this we can extract either distinct proper-time maximizing segments linking $\left(x_{0}, y_{0}\right)$, or a Jacobi field which shows $x_{0}$ to be conjugate to $y_{0}$. In either case $\left(x_{0}, y_{0}\right) \in \operatorname{sing}(\ell)$. 
Concerning smoothness of $\ell$ : since points near $\left(x_{0}, y_{0}\right) \notin \operatorname{sing}(\ell)$ are timelike separated but not conjugate, the inverse function theorem guarantees $(x, v) \mapsto\left(x, \exp _{x} v\right)$ acts as a smooth diffeomorphism near $\left(x_{0}, v_{0}\right):=$ $\left(x_{0}, \exp _{x}^{-1} y_{0}\right)$, as does $(y, w) \mapsto\left(y, \exp _{y} w\right)$ near $\left(y_{0}, \exp ^{-1} x_{0}\right)$ [ibid, pp 314328]. From $(d)$, we deduce $\ell$ is differentiable near $\left(x_{0}, y_{0}\right)$ and its gradient

$$
-D \ell(x, y)=\left.\left(\frac{v_{*}}{\left|v_{*}\right| g}, \frac{w_{*}}{\left|w_{*}\right|_{g}}\right)\right|_{(v, w)=\left(\exp _{x}^{-1} y, \exp _{y}^{-1} x\right)}
$$

depends smoothly on $(x, y)$ there. Thus $\ell(x, y)$ is smooth near $\left(x_{0}, y_{0}\right)$.

We also claim $\ell$ fails to be smooth at each $\left(x_{0}, y_{0}\right) \in \operatorname{sing}(\ell)$. If $\ell\left(x_{0}, y_{0}\right)=$ $-\infty$ this is obvious since smooth functions are by definition real-valued. If $\ell\left(x_{0}, y_{0}\right)=0$, we will show in (e) below that differentiability of $\ell$ fails unless $x_{0}=y_{0}$, in which case $\ell(x, y)=+\infty$ for points arbitrarily close to $\left(x_{0}, y_{0}\right)$. If $\ell\left(x_{0}, y_{0}\right)>0$ then we are on the timelike cut locus where Theorem 3.5 shows $\ell$ fails to be $C^{1,1}$ smooth.

(e) Suppose $\ell(x, y)=0$. Let $X \subset M$ denote a convex neighbourhood of $x$, meaning for each $z \in X$, the inverse map to $\exp _{z}: T_{z} M \longrightarrow M$ acts diffeomorphically on $X$, as in e.g. [77, §5.7]. Let $\sigma:[0,1] \longrightarrow M$ be a (null) action minimizing segment joining $x$ to $y$. For $s>0$ sufficiently small that $z:=\sigma(s) \in X$ we find

$$
\begin{aligned}
\ell\left(\exp _{x} v, z\right) & =\ell\left(\exp _{z} \circ\left(\exp _{z}\right)^{-1} \circ \exp _{x} v, z\right) \\
& =-L\left(-\left(\exp _{z}\right)^{-1} \circ \exp _{x} v, z ; 1\right) \\
& =-L\left(\dot{\sigma}(s)-\left(D \exp _{z}^{-1}\right)_{\dot{\sigma}(s)}\left(D \exp _{x}\right)_{\mathbf{0}} v, z ; 1\right) .
\end{aligned}
$$

Now if $\ell(\cdot, y)$ has a supergradient $w \in T_{x}^{*} M$, the reverse triangle inequality yields

$$
\begin{aligned}
\ell\left(\exp _{x} v, z\right) & \leq \ell\left(\exp _{x} v, y\right)-\ell(z, y) \\
& \leq \ell(x, y)-\ell(z, y)+w[v]+o\left(|v|_{\tilde{g}}\right)
\end{aligned}
$$

as $v \rightarrow 0$. Since $\ell(x, y)=\ell(z, y)=L(\dot{\sigma}(s) ; 1)=0$, this would imply subdifferentiability of $L(\cdot, z ; 1)$ at $\dot{\sigma}(s)$ - a contradiction with Corollary 3.2 unless $\dot{\sigma}(s)=0$, in which case $x=y=z$, both derivatives in (29) are given by the identity map, and $H(w ; 1)=0$.

Corollary 3.7 (Twist and non-degeneracy) Fix $0<q<1$. Then (i) $\ell^{q}$ inherits properties (a)-(d) of Theorem 3.6 from $\ell$. (ii) If $\frac{1}{q} \ell(\cdot, y)^{q}$ has 
supergradient $w$ at $x$ then $y=\exp _{x} D H(w, x ; q)$, where $H$ is defined at (23)). (iii) If $(x, y) \notin \operatorname{sing}(\ell)$ then $\operatorname{det} \frac{\partial^{2}}{\partial x^{j} \partial y^{i}} \ell^{q}(x, y) \neq 0$. (iv) If $(x, y) \in\{\ell>$ $0\} \cap \operatorname{sing}(\ell)$ then

$$
\sup _{0<|w|_{\tilde{g}}<1} \frac{\ell^{q}\left(\exp _{x}^{\tilde{g}} w, y\right)+\ell^{q}\left(\exp _{x}^{\tilde{g}}-w, y\right)-2 \ell^{q}(x, y)}{2|w|_{\tilde{g}}^{2}}=+\infty
$$

Proof. (i) For a function $u: \mathbf{R}^{n} \longrightarrow[0, \infty)$ to have semiconvexity constant $C$ at $\bar{x}$ is equivalent to asserting $p \in \partial . u(\bar{x})$ non-empty and

$$
u(x) \geq u(\bar{x})+p[x-\bar{x}]-\frac{1}{2} C|x-\bar{x}|^{2}+o\left(|x-\bar{x}|^{2}\right)
$$

as $x \rightarrow \bar{x}$, for all $\bar{x}$ near $\bar{x}$. Raising this inequality to exponent $q$, for $|t|<1$ the existence of $\left|t_{*}\right| \leq|t|$ such that

$$
(1+t)^{q}=1+q t+q(q-1) t^{2} / 2+q(q-1)(q-2) t_{*}^{3} / 6
$$

shows $u^{q} / q$ inherits semiconvexity constant $C u^{q-1}+2(1-q) u^{q-2}|D u|^{2}$ at $\bar{x}$ from $u$. Applying this argument in Riemannian normal coordinates establishes semiconvexity of the locally Lipschitz function $u^{q}(\cdot):=\ell^{q}(\cdot, y)$ at each point $\bar{x}$ with $\ell(\bar{x}, y)>0$ in view of Theorem $3.6(d)$. The remaining properties (a)-(d) follow from the one-sided chain rule [67, Lemma 5] and our convention $(-\infty)^{1 / q}:=-\infty=:(-\infty)^{q}$. We shall obtain a strengthening of (e) in the course of proving (ii) below: namely, that $\ell(x, y)=0$ implies the superdifferential of $\frac{1}{q} \ell(\cdot, y)^{q}$ at $x$ is empty.

(iv) The alternative to (30) is that $u(\cdot):=\ell^{q}(\cdot, y)$ has semiconcavity constant $C<\infty$ at some $\bar{x}$ with $(\bar{x}, y) \in\{\ell>0\} \cap \operatorname{sing}(\ell)$. The same argument as above then implies $u^{1 / q}$ has semiconcavity constant $C u^{\frac{1}{q}-1}+$ $2\left(\frac{1}{q}-1\right) u^{\frac{1}{q}-2}|D u|^{2}$ at $\bar{x}$, contradicting Theorem 3.5. So (30) must hold.

(ii) If $\frac{1}{q} \ell(\cdot, y)^{q}$ admits $w \in T_{x} M$ as a supergradient, then $\ell(\cdot, y)$ admits $\ell(x, y)^{1-q} w$ as a supergradient at $x$, by the (one-sided) chain rule. When $\ell(x, y)=0$ this contradicts Theorem 3.6(e) whether or not $x$ is distinct from $y$, since $H(\mathbf{0} ; 1)=+\infty \neq 0$. Thus $\ell(x, y)>0$. Now (c) implies differentiability of $\frac{1}{q} \ell(\cdot, y)$ at $x$, with

$$
w:=D_{x} \ell^{q}(x, y) / q=\left.|v|_{g}^{q-2} v_{*}\right|_{v=-\exp _{x}^{-1} y} .
$$


Thus $y=\exp _{x}-|w|_{g}^{q^{\prime}-2} w=\exp _{x} D H(w, x ; q)$ is uniquely determined by $x$ and $w$, where $\frac{1}{q}+\frac{1}{q^{\prime}}=1$.

(iii) Now fix $(x, y) \notin \operatorname{sing}(\ell)$. Differentiating (31) with respect to $y$ yields

$$
-D_{y x}^{2} \ell(x, y) / q=\left.\frac{|v|^{2} g-(2-q) v_{*} \otimes v_{*}}{|v|_{g}^{4-q}}\right|_{v=\exp _{x}^{-1} y} D_{y}\left(\exp _{x}^{-1} y\right) .
$$

Our choice $(+-\ldots-)$ of signature for $g$ shows the first factor is negative definite when $q<1$ since $v$ is timelike; the second factor has non-zero determinant since $y$ is in the chronological future but not in the conjugate locus of $x$.

Definition 3.8 (Approximate differentiability) $A$ map $F: M \longrightarrow N$ between differentiable manifolds is approximately differentiable at $x \in M$ if there exists a map $\tilde{F}: M \longrightarrow N$, differentiable at $x$, such that the set $\{\tilde{F} \neq F\}$ has zero density at $x$, i.e.

$$
\lim _{r \rightarrow 0} \frac{\operatorname{vol}\left[\left\{x \in B_{r}(x) \mid F(x) \neq \tilde{F}(x)\right\}\right]}{\operatorname{vol}\left[B_{r}(x)\right]}=0,
$$

where the radius $r$ and vol refers to the coordinate radius and volume in any (and hence all) coordinate system(s) at $x$.

A well-known result of Aleksandrov [3] whose Riemannian version [17] we shall exploit asserts that convex (and hence semiconvex) functions have approximate second derivatives almost everywhere. In fact, more is true: any semiconvex function agrees with a $C^{2}$ function, outside a set of arbitrarily small volume. We shall make use of this Lusin style approximation result, which follows from the fact that convex gradients are countably Lipschitz, e.g. [6] [82], and the analogous $C^{1}$ approximation result for Lipschitz functions, e.g. $\S 6.6$ of [40].

\section{Kantorovich duality with Lorentz distance}

To characterize the $q$-geodesics defined above, we must first study the optimization (6), which is a Monge-Kantorovich optimal transportation problem. As an infinite-dimensional linear program, it is well-known to have the following dual problem, provided the infinum is finite as described e.g. in [92]:

$$
\frac{1}{q} \ell_{q}(\mu, \nu)^{q}=\inf \left\{\int_{M} u d \mu+\int_{M} v d \nu \mid \frac{1}{q} \ell^{q} \leq u \oplus v \in L^{1}(\mu \times \nu)\right\},
$$


where

$$
(u \oplus v)(x, y):=u(x)+v(y)
$$

and $u$ and $v$ are lower semicontinuous. Moreover, given any sets $X \supset \operatorname{spt} \mu$ and $Y \supset$ spt $\nu$, we may restrict the infimum (33) to pairs of functions $u=v_{q}$ on $X$ and $v=u_{\tilde{q}}$ on $Y$, where

$$
\begin{aligned}
& v_{q}(x):=\sup _{y \in Y} \frac{1}{q} \ell^{q}(x, y)-v(y) \\
& u_{\tilde{q}}(y):=\sup _{x \in X} \frac{1}{q} \ell^{q}(x, y)-u(x) .
\end{aligned}
$$

Such pairs of functions $(u, v)=\left(v_{q}, u_{\tilde{q}}\right)$ are called $\frac{\ell^{q}}{q}$-convex or $\frac{\ell^{q}}{q}$-conjugate. Notice however, that these notions depend on the choice of sets $X$ and $Y$.

Unfortunately, since the function $\ell$ jumps to $-\infty$ outside the causal future, it is not clear whether the infimum (33) is generally attained. However, we shortly show that it will be attained when the measures $\mu$ and $\nu$ satisfy the following condition.

Definition 4.1 ( $q$-separated) Fix $0<q \leq 1$. We say $(\mu, \nu) \in \mathcal{P}_{c}(M)^{2}$ is $q$-separated by $\pi \in \Pi(\mu, \nu)$ and lower semicontinuous $u$ : spt $\mu \longrightarrow \mathbf{R} \cup\{+\infty\}$ and $v: \operatorname{spt} \nu \longrightarrow \mathbf{R} \cup\{+\infty\}$ if

$$
u(x)+v(y) \geq \frac{1}{q} \ell(x, y)^{q} \quad \forall(x, y) \in \operatorname{spt}[\mu \times \nu],
$$

$\operatorname{spt} \pi \subset S:=\left\{(x, y) \in \operatorname{spt}[\mu \times \nu] \mid u(x)+v(y)=\frac{1}{q} \ell(x, y)^{q}\right\}$ and $S \subset\{\ell>0\}$.

Remark 4.2 Compactness of $S$ and its disjointness from $\{\ell \leq 0\}$ are essential to this definition: $\ell>0$ on $S$ shows events described by $d \mu(x)$ and $d \nu(y)$ can be matched so that each $x$ lies in the chronological - as opposed to the causal - past of its assigned $y$. One can also strengthen the definition of q-separation by requiring disjointness of $S$ from $\operatorname{sing}(\ell)$. This leads to a simpler variant of the theory, but one which is unable to rule out transportation to the timelike cut locus. This limitation is unsatisfactory in the context of our intended application of these developments to nonsmooth spacetimes.

Although mildly restrictive, this definition has the following theorem as an immediate consequence, which allows us to circumvent various subtleties involving non-compact support and/or null geodesics. In Section $\$ 7$ we relax this restriction by approximation. Of course, it may turn out that the dual 
is actually attained in this greater generality, as in the Riemannian case 41 . Ideas of Bertrand, Pratelli, Puel [27] 26] and Suhr [88 may prove relevant to this question, as may my own work with Puel [69].

A set $S \subset M \times M$ is called $\ell^{q}$-cyclically monotone, if for each $k \in \mathbf{N}$, permutation $\sigma$ on $k$ letters, for each $\left(x_{1}, y_{1}\right), \ldots,\left(x_{k}, y_{k}\right) \in S$ we find

$$
\sum_{i=1}^{k} \ell\left(x_{i}, y_{i}\right)^{q} \geq \sum_{i=1}^{k} \ell\left(x_{i}, y_{\sigma(i)}\right)^{q} .
$$

This property is well-known to characterize the support of $\ell^{q}$-optimal measures $\pi$ for (6), provided the set where the cost is not finite is closed [20; $\ell^{q}$-monotonicity refers to the case $k=2$ in (36).

Theorem 4.3 (Duality by $q$-separation) Fix $0<q \leq 1$. If $(\mu, \nu) \in$ $\mathcal{P}_{c}(M)^{2}$ is q-separated by $(\pi, u, v)$, then $(i)(u, v)=\left(v_{q}, u_{\tilde{q}}\right)$ on $X \times Y:=$ $\operatorname{spt}[\mu \times \nu]$ in (34). (ii) The set $S=\left\{(x, y) \in X \times Y \mid u \oplus v=\frac{1}{q} \ell^{q}\right\}$ is compact and $\ell^{q}$-cyclically monotone. (iii) The potentials $(u, v)$ minimize (33), while $\pi$ maximizes (66). (iv) The extensions $u:=v_{q}$ and $v:=u_{\tilde{q}}$ are semiconvex Lipschitz functions on neighbourhoods of $X$ and $Y$, respectively, with Lipschitz and semiconvexity constants estimated by those of $\frac{1}{q} \ell^{q}$ on $S$.

Proof. (i) Let $(\mu, \nu) \in \mathcal{P}_{c}(M)^{2}$ be $q$-separated by $(\pi, u, v)$. For $\bar{x} \in M$ the supremum

$$
v_{q}(\bar{x}):=\sup _{y \in Y} \frac{1}{q} \ell^{q}(\bar{x}, y)-v(y)
$$

is attained, due to the compactness of $Y:=\operatorname{spt} \nu$ and the upper semicontinuity assumed for $-v$ and established for of $\ell^{q}$ in Corollary 3.7. If $\bar{x} \in X$ then $u(\bar{x}) \geq v_{q}(\bar{x})$ follows from the definition of $q$-separation. In this case the existence of $\bar{y} \in Y$ such that $(\bar{x}, \bar{y}) \in \operatorname{spt} \pi \subset S:=\{(x, y) \in X \times Y \mid$ $\left.u(x)+v(y)=\frac{1}{q} \ell(x, y)^{q}\right\}$ follows from compactness of $X:=\operatorname{spt} \mu$ and $Y$. This $\bar{y}$ must then maximize (37), and $S \subset\{\ell>0\}$ shows $u(\bar{x})=v_{q}(\bar{x})$ to be finite on $X$; since $u$ was not defined outside $X$ we may take $u:=v_{q}$ as a definition there. The identity $v=u_{\tilde{q}}$ is proved similarly.

(ii) Compactness of $X, Y$ and the lower semicontinuity of $u \oplus v-\frac{1}{q} \ell^{q}$ asserted by Corollary 3.7 show that $S$ is compact. Choosing any $k \in \mathbf{N}$ and 
a permutation $\sigma$ on $k$ letters, for each $\left(x_{1}, y_{1}\right), \ldots,\left(x_{k}, y_{k}\right) \in S$ we find

$$
\begin{aligned}
\frac{1}{q} \sum_{i=1}^{k} \ell\left(x_{i}, y_{i}\right)^{q} & =\sum_{i=1}^{k} u\left(x_{i}\right)+v\left(y_{i}\right) \\
& =\sum_{i=1}^{k} u\left(x_{i}\right)+v\left(y_{\sigma(i)}\right) \\
& \geq \frac{1}{q} \sum_{i=1}^{k} \ell\left(x_{i}, y_{\sigma(i)}\right)^{q}
\end{aligned}
$$

as desired.

(iii) Since $S$ is compact, Corollary 3.7 shows $\ell$ is bounded above on $S$. Being lower semicontinuous, $u$ and $v$ are bounded below on $S$. Because $\pi$ vanishes outside $S$,

$$
\begin{aligned}
\frac{1}{q} \int_{M^{2}} \ell(x, y)^{q} d \pi(x, y) & =\int_{M^{2}}[u(x)+v(y)] d \pi(x, y) \\
& =\int_{M} u d \mu+\int_{M} v d \nu
\end{aligned}
$$

where the second equality follows from the fact that $\pi \in \Pi(\mu, \nu)$ has $\mu$ and $\nu$ for its left and right marginals. Observing that the inequality $\frac{1}{q} \ell_{q}(\mu, \nu)^{q} \leq \inf$ of (33) is elementary to derive makes it clear that $(u, v)$ attain the infimum and $\pi$ attains the maximum (6).

(iv) Compactness of $S$ shows its (Riemannian) distance $3 R:=d_{\tilde{g} \oplus \tilde{g}}(\{\ell \leq$ $0\}, S)$ from $\{\ell \leq 0\}$ is positive. Given $r \geq 0$, let $X_{r}:=\left\{x \in M \mid d_{\tilde{g}}(x, X) \leq\right.$ $r\}$ denote the set of points whose Riemannian distance from $X$ is at most $r$. Define $Y_{r} \subset M$ and $S_{r} \subset M^{2}$ analogously. According to Theorem 3.6 the restriction of $\ell^{q} / q$ to the Riemannian neighbourhour $S_{2 R}$ of size $2 R$ around $S$ is Lipschitz and has semiconvexity constant $C_{2 R}>-\infty$.

We claim the (Riemannian) Lipschitz constant of $v_{q}$ on some sufficiently small neighbourhood $X_{r}$ of $X=\operatorname{spt} \mu$ is no greater than $\|\ell\|_{C^{0,1}\left(S_{2 R}\right)}$. However, let us first establish lower semicontinuity (38) of $v_{q}$ at each point $\bar{x} \in X$. Fixing $\bar{x} \in X$, there exists $\bar{y}$ with $(\bar{x}, \bar{y}) \in S$ as above. Letting $x \in B_{R}(\bar{x})$, 
we find

$$
\begin{aligned}
v_{q}(x) & \geq \ell(x, \bar{y} ; q)-v(\bar{y}) \\
& \geq \ell(\bar{x}, \bar{y} ; q)-\|\ell(\cdot, \bar{y} ; q)\|_{C^{0,1}\left(B_{R}(\bar{x})\right)} d_{\tilde{g}}(x, \bar{x})-v(\bar{y}) \\
& \geq v_{q}(\bar{x})-\frac{1}{q}\left\|\ell^{q}\right\|_{C^{0,1}\left(S_{R}\right)} d_{\tilde{g}}(x, \bar{x}) .
\end{aligned}
$$

If $x \in X$ we can interchange $\bar{x}$ with $x$ to obtain the desired Lipschitz bound for $v_{q}$ on $X$ (and for $u_{\tilde{q}}$ on $Y$ ), though not yet on $X_{r}$ (or $Y_{r}$, respectively). However, even for $x \in X_{r} \backslash X$ we deduce the desired lower semicontinuity:

$$
\lim \inf _{x \rightarrow \bar{x}} v_{q}(x) \geq v_{q}(\bar{x}) .
$$

Taking $r>0$ sufficiently small ensures that $X_{r}$ inherits compactness from $X$ (and $Y_{r}$ from $Y$ ). Taking $r>0$ smaller still ensures $S_{(r, 0)}:=\{(x, y) \in$ $\left.X_{r} \times Y \mid v_{q}(x)+v(y)=\ell^{q}(x, y) / q\right\}$ is contained in $S_{R}$. If not, there exists a sequence $\left(x_{k}, y_{k}\right) \in S_{(1 / k, 0)} \backslash S_{R}$ with convergent subsequence. Lower semicontinuity of $v$ on $Y$, Theorem 3.6 and (38) imply the limit $\left(x_{\infty}, y_{\infty}\right)$ belongs to $S$. But this contradicts $\left(x_{k}, y_{k}\right) \notin S_{R}$.

Now apply the preceding argument to an arbitrary pair of points $\bar{x} \in X_{r}$ and $x \in B_{R}(\bar{x}) \cap X_{r}$. As before there exists $\bar{y}$ with $(\bar{x}, \bar{y}) \in S_{(r, 0)} \subset S_{R}$, and

$$
\begin{aligned}
v_{q}(x) & \geq \ell(x, \bar{y} ; q)-v(\bar{y}) \\
& \geq \ell(\bar{x}, \bar{y} ; q)-\|\ell(\cdot, \cdot ; q)\|_{C^{1}\left(B_{R}(\bar{x}, \bar{y})\right)} d_{\tilde{g}}(x, \bar{x})-v(\bar{y}) \\
& =v_{q}(\bar{x})-\frac{1}{q}\left\|\ell^{q}\right\|_{C^{1}\left(S_{2 R}\right)} d_{\tilde{g}}(x, \bar{x})
\end{aligned}
$$

interchanging $\bar{x}$ with $x$ yields the desired Lipschitz bound for $v_{q}$ on $X_{r}$.

Finally, for $|w|_{\tilde{g}}<R$ and $(\bar{x}, \bar{y}) \in S_{(r, 0)} \subset S_{R}$ as above, the Riemannian exponential yields

$$
\begin{aligned}
& v_{q}\left(\exp _{\bar{x}}^{\tilde{g}} w\right)+v_{q}\left(\exp _{\bar{x}}^{\tilde{g}}-w\right)-2 v_{q}(\bar{x}) \\
\geq & \ell\left(\exp _{\bar{x}}^{\tilde{g}} w, \bar{y} ; q\right)+\ell\left(\exp _{\bar{x}}^{\tilde{g}}-w, \bar{y} ; q\right)-2 \ell(\bar{x}, \bar{y} ; q) \\
\geq & C_{2 R}|w|_{\tilde{g}}^{2} .
\end{aligned}
$$

This shows the semiconvexity of $v_{q}$ on $X_{r}$. Similarly, taking $r>0$ small enough yields $u_{\tilde{q}}$ semiconvex and Lipschitz on $Y_{r}$.

The following lemma shows the notion of $q$-separation is not vacuous; instead it puts us back into the more standard framework of optimal transportation with respect to uniformly continuous cost functions. 
Lemma 4.4 (Existence of $q$-separation) Fix $0<q \leq 1$ and $\mu, \nu \in \mathcal{P}_{c}(M)$. If $\operatorname{spt}[\mu \times \nu] \subset M \times M \backslash\{\ell \leq 0\}$ then $(\mu, \nu)$ is q-separated.

Proof. Theorem 3.6 asserts continuity of $\ell$ on the compact set $X \times Y:=$ $\operatorname{spt}[\mu \times \nu]$. In this case, the supremum (66) and infimum (33) are well-known to be attained by $\pi \in \Pi(\mu, \nu)$ and a pair of uniformly continuous functions $(u, v)=\left(v_{q}, u_{\tilde{q}}\right)$ satisfying (34), e.g. 82, 92. Duality (33) implies spt $\pi$ is contained in the equality set $S \subset X \times Y$ for $u \oplus v-\frac{1}{q} \ell^{q} \geq 0$ - which inherits both compactness and disjointness to $\operatorname{sing}(\ell)$ from $X \times Y$.

\section{Characterizing $q$-geodesics via duality}

Armed with a duality theory for $q$-separated probability measures $\left(\mu_{0}, \mu_{1}\right)$, we now turn to the analytical characteristics of the $q$-geodesic $s \in[0,1] \mapsto$ $\mu_{s} \in \mathcal{P}_{c}(M)^{2}$ which links them. These can in principal be described using either an Eulerian [22] [78] [29] [9] [83] or Lagrangian framework [92]. Here we employ a Lagrangian approach consistent with the analogous results originally obtained in Euclidean space [66] and, with Cordero-Erausquin and Schmuckenschläger, on Riemannian manifolds [67] [36] [37]. For the case $q=1$ not covered here, Suhr develops a different approach, based on dynamical transport plans (i.e. measures on the space of geodesic segments) [88.

Take $M$ to be a globally hyperbolic spacetime, $N=M \times M$ and $0<q<1$. When $\mu_{0}, \mu_{1} \in \mathcal{P}_{c}(M)$ are $q$-separated by $(\pi, u, v)$ and $\mu_{0} \in \mathcal{P}_{c}^{a c}(M)$, one task will be to show $\pi=\left(i d \times F_{1}\right)_{\#} \mu_{0}$ where $F_{s}(x):=\exp _{x}(s D H(D u(x), x ; q))$ for each $s \in[0,1]$ and $i d: M \longrightarrow M$ denotes the identity map $i d(x)=x$ on $M$. In other words, the $\ell^{q}$-optimal coupling $\pi \in \Pi\left(\mu_{0}, \mu_{1}\right)$ from Theorem 4.3 concentrates its mass on the graph of a map $F_{1}: M \longrightarrow M$. By analogy with the Euclidean case [71], such a map is said to solve Monge's problem [82] [92]. This is accomplished in Theorem 5.8, which also characterizes the unique coupling achieving the maximum (6), and is the analog of Brenier's theorem from the Euclidean setting [28] 65] and my Riemannian generalization [67]. Its corollaries go further by showing $\mu_{s}:=F_{s \#} \mu_{0}$ is the unique $q$-geodesic with the given endpoints, that it is absolutely continuous with respect to $\operatorname{vol}_{g}$ and that its density $\rho_{s}:=d \mu_{s} / d \operatorname{vol}_{g}$, is related to the Jacobian $J F_{s}(x)$ of $F_{s}$ by the Monge-Ampère type equation

$$
\rho_{0}(x)=\rho_{s}\left(F_{s}(x)\right) J F_{s}(x) \quad \mu_{0} \text {-a.e., }
$$


whenever $s<1$ or $\mu_{1} \in \mathcal{P}_{c}^{a c}(M)$. In particular, the Lagrangian path description of the worldlines of the individual events making up this geodesic is given by the map $(x, s) \in M \times[0,1] \mapsto F_{s}(x)$.

To achieve this description we will need to establish various analytical properties of $F_{s}$ along the way, such as the fact that $F_{s}^{-1}$ is (Lipschitz) continuous for each $s<1$ in Theorem 5.3. Similarly, the fact that $F_{s}$ is countably Lipschitz follows from Theorem 4.3(iv), allowing us to make sense of its Jacobian $J F_{s}(x):=\left|\operatorname{det} \tilde{D} F_{s}(x)\right|$ almost everywhere. The Monge-Ampère type equation (39) will be the key to analyzing convexity properties of the Boltzmann-Shannon or relative entropy $e(s):=E_{V}\left(\mu_{s}\right)$ along the $q$-geodesic in question, so we will need to be able to compute two derivatives of (39) with respect to $s$. Fortunately, $s \in[0,1] \mapsto F_{s}(x)$ is a proper-time maximizing segment for each $x \in$ Dom $D u$, so the derivatives desired can be computed using Jacobi fields. This is accomplished in Lemma 5.7, where we see the first Lorentzian connection linking optimal transport to semi-Riemannian curvature.

We begin by showing that if an $\ell^{q}$-optimal measure $\pi$ couples two distinct pairs of events, i.e. $(x, y),\left(x^{\prime}, y^{\prime}\right) \in \operatorname{spt} \pi$, then the midpoint $\bar{z}_{1 / 2}(x, y)$ of a proper-time maximizing geodesic segment joining $x$ to $y$ cannot coincide with the midpoint of any proper-time maximizing geodesic segment joining $x^{\prime}$ to $y^{\prime}$. Similarly $\bar{z}_{s}(x, y) \neq \bar{z}_{s}\left(x^{\prime}, y^{\prime}\right)$ for $0<s<1$. Such pairs of coupled events satisfy (40) by Theorem 4.3(ii).

Proposition 5.1 (Lagrangian trajectories don't cross) Fix $q, s \in(0,1)$. If $Z_{s}(x, y)$ from (17) intersects $Z_{s}\left(x^{\prime}, y^{\prime}\right)$ yet

$$
\ell\left(x, y^{\prime}\right)^{q}+\ell\left(x^{\prime}, y\right)^{q} \leq \ell(x, y)^{q}+\ell\left(x^{\prime}, y^{\prime}\right)^{q}
$$

then $(x, y)=\left(x^{\prime}, y^{\prime}\right)$.

Proof. This argument is inspired by the Riemannian case [36]. The reverse triangle inequality and strict concavity of $r \mapsto r^{q}$ assert

$$
\begin{aligned}
\ell\left(x, y^{\prime}\right)^{q} & \geq\left(s \frac{\ell(x, m)}{s}+(1-s) \frac{\ell\left(m, y^{\prime}\right)}{1-s}\right)^{q} \\
& \geq s^{1-q} \ell(x, m)^{q}+(1-s)^{1-q} \ell\left(m, y^{\prime}\right)^{q} \\
& =s \ell(x, y)^{q}+(1-s) \ell\left(x^{\prime}, y^{\prime}\right)^{q} .
\end{aligned}
$$

The first inequality is strict unless $m$ lies on an action minimizing segment joining $x$ to $y^{\prime}$ - or equivalently $y^{\prime}$ lies beyond $m$ on the unique future 
directed geodesic from $x$ passing through $m$; the second inequality is strict unless $\frac{\ell(x, m)}{s}=\frac{\ell\left(m, y^{\prime}\right)}{1-s}$, or equivalently $\ell(x, y)=\ell\left(x^{\prime}, y^{\prime}\right)$. Similarly,

$$
\begin{aligned}
\ell\left(x^{\prime}, y\right)^{q} & \geq\left(s \frac{\ell\left(x^{\prime}, m\right)}{s}+(1-s) \frac{\ell(m, y)}{1-s}\right)^{q} \\
& \geq s \ell\left(x^{\prime}, y^{\prime}\right)^{q}+(1-s) \ell(x, y)^{q},
\end{aligned}
$$

and at least one of these two inequalities is strict unless $\ell(x, y)=\ell\left(x^{\prime}, y^{\prime}\right)$ and $y$ lies beyond $m$ on the extension of the geodesic from $x^{\prime}$ through $m$.

Summing these contradicts (40) unless equalities hold throughout. But this forces $\ell(x, y)=\ell\left(x^{\prime}, y^{\prime}\right)$ and all five points $x, x^{\prime}, m, y^{\prime}, y$ onto the same timelike geodesic, with $x$ and $x^{\prime}$ in the past of $m$ and $y$ and $y^{\prime}$ in its future. Since the segments $x y$ and $x^{\prime} y^{\prime}$ of this geodesic have the same proper time and $m$ divides them both in the same ratio, we conclude $x=x^{\prime}$ and $y=y^{\prime}$. as desired.

Corollary 5.2 (Continuous inverse maps) Fix q, $s \in(0,1)$. If $\left(\mu_{0}, \mu_{1}\right) \in$ $\mathcal{P}_{c}(M)^{2}$ is q-separated and $X_{i}:=$ spt $\mu_{i}$, there is a continuous map $W$ : Dom $W \subset M \longrightarrow S \subset X_{0} \times X_{1}$ such that if $\mu_{s}$ lies on a q-geodesic (8) then $W_{\#} \mu_{s}$ maximizes $\ell^{q}$ in $\Pi\left(\mu_{0}, \mu_{1}\right)$. Here $\operatorname{Dom} W=Z_{s}(S)$ where $Z_{s}$ is from (16) and $S$ from the Definition 4.1 of $q$-separated. Moreover, $\bar{z}_{s} \circ W$ acts as the identity map on $\bar{z}_{s}(S)$ whenever $\bar{z}_{s}$ is consistent with Lemma 2.8 .

Proof. Duality (33) holds with continuous semiconvex optimizers $(u, v)=$ $\left(v_{q}, u_{\tilde{q}}\right)$ according to Theorem 4.3, which also shows the set $S=\{(x, y) \in$ $\left.X_{0} \times X_{1} \mid u(x)+v(y)=\ell(x, y ; q)\right\}$ to be compact and $\ell^{q}$-cyclically monotone. Recall $q$-separation requires $S$ to be disjoint from $\{\ell \leq 0\}$. Let $m_{k} \in Z_{s}\left(x_{k}, y_{k}\right)$ for some sequence $\left(x_{k}, y_{k}\right) \in S$. where $Z_{s}(x, y)$ is from (17). Assume $m_{k} \rightarrow m$, and extract a subsequential limit $\left(x_{k(j)}, y_{k(j)}\right) \rightarrow(x, y)$ using compactness of $S$. Then $m \in Z_{s}(x, y)$. Similarly, if another subsequence of $\left(x_{k}, y_{k}\right)$ converges to a different limit $\left(x^{\prime}, y^{\prime}\right) \in S$ then $m \in Z_{s}\left(x^{\prime}, y^{\prime}\right)$. Thus $Z_{s}(x, y)$ intersects $Z_{s}\left(x^{\prime}, y^{\prime}\right)$. The $\ell^{q}$-cylical monotonicity of $S$ implies (40), which forces $(x, y)=\left(x^{\prime}, y^{\prime}\right)$ according to Proposition 5.1. This means $W(m):=(x, y) \in X_{0} \times X_{1}$ is well-defined and continuous, since all subsequences of $\left(x_{k}, y_{k}\right)=W\left(m_{k}\right)$ converge to the same limit $(x, y)=W(m)$. Moreover, $W$ acts as a right-inverse for $\bar{z}_{s}$ on $\bar{z}_{s}(S)$.

Now let $\mu_{s}$ satisfy (8). Since $q$-separation yields $0<\ell_{q}\left(\mu_{0}, \mu_{1}\right)<\infty$, Proposition 2.9 provides $\omega \in \mathcal{P}\left(M^{3}\right)$ with marginals $\left(\mu_{0}, \mu_{s}, \mu_{1}\right)$ whose projection onto any pair of coordinates is $\ell^{q}$-optimal and has $z \in Z_{s}(x, y)$ for 
$\omega$-a.e. $(x, z, y)$. In particular $\pi=\operatorname{proj}_{13 \#} \omega$ maximizes $\ell^{q}$ on $\Pi\left(\mu_{0}, \mu_{1}\right)$, hence is supported in the compact set $S$ according to Theorem 4.3 and the duality (33). It follows that $\mu_{s}=\operatorname{proj}_{2} \omega$ vanishes outside Dom $W:=Z_{s}(S)$, which is compact according to Lemma 2.5. Denoting $(X(m), Y(m)):=W(m)$, the preceding paragraph shows $\omega$ to vanish outside the graph of $W$. Thus $\omega=(X \times i d \times Y)_{\#} \mu_{s}$ by e.g. Lemma 3.1 of [2], hence $\pi=W_{\#} \mu_{s}$ as desired.

The continuous map $W$ of the preceding corollary is actually Lipschitz:

Theorem 5.3 (Lipschitz inverse maps) Under the hypotheses of Corollary 5.2, the map $W: Z_{s}(S) \longrightarrow M^{2}$ defined in that corollary is Lipschitz continuous with respect to any fixed choice of Riemannian distance $d_{\tilde{g}}$ on $M$.

To avoid interrupting the flow of ideas, we defer the discussion and rather technical proof of Theorem 5.3 to Appendix $\mathrm{A}$.

Lemma 5.4 (Variational characterization of geodesic endpoints) Fix $0<q<1$ and a timelike proper-time maximizing segment $s \in[0,1] \mapsto x_{s} \in$ $M$. For each $0<s<1$ and $x \in M$,

$$
\ell\left(x, x_{1}\right)^{q} \geq s^{1-q} \ell\left(x, x_{s}\right)^{q}+(1-s)^{1-q} \ell\left(x_{s}, x_{1}\right)^{q}
$$

with equality if and only if $x=x_{0}$.

Proof. The reverse triangle inequality yields

$$
\ell\left(x, x_{1}\right) \geq s \frac{\ell\left(x, x_{s}\right)}{s}+(1-s) \frac{\ell\left(x_{s}, 1\right)}{1-s},
$$

with equality only if $x_{s}$ lies on the minimizing segment joining $x$ to $x_{1}$. In other words, equality holds only if $x$ lies beyond $x_{s}$ on the past-directed geodesic from $x_{1}$ through $x_{s}$ (this geodesic is unique since $x_{s}$ is internal to the minimizing segment joining $x_{0}$ to $x_{1}$ ). Strict concavity of the function $r \mapsto r^{q}$ yields (41), with equality forcing

$$
\frac{\ell\left(x, x_{s}\right)}{s}=\frac{\ell\left(x_{s}, 1\right)}{1-s} .
$$

This equation is uniquely solved on the geodesic in question by $x=x_{0}$.

The following proposition shows our $q$-separation property propogates from the endpoints to the interior of a $q$-geodesic. 
Proposition 5.5 (Star-shapedness of $q$-separation) Fix $0<q<1$. If $s \in[0,1] \mapsto \mu_{s} \in \mathcal{P}_{c}(M)$ is a q-geodesic and $\left(\mu_{0}, \mu_{1}\right)$ is q-separated, then $\left(\mu_{s}, \mu_{t}\right)$ is q-separated for all $0 \leq s<t \leq 1$.

Proof. Let $\left(\mu_{0}, \mu_{1}\right)$ be $q$-separated by $\pi$ and $(u, v)$. Since every subsegment of a $q$-geodesic is itself a $q$-geodesic (after affine reparameterization), it suffices to prove $\left(\mu_{0}, \mu_{s}\right)$ and $\left(\mu_{t}, \mu_{1}\right)$ are $q$-separated. We show this for $\left(\mu_{0}, \mu_{s}\right)$; the proof for $\left(\mu_{t}, \mu_{1}\right)$ is similar.

Setting $X_{s}:=\operatorname{spt} \mu_{s}$, Theorem 4.3 asserts that $u$ and $v$ are continuous on $X_{0}$ and $X_{1}$ respectively, $S:=\left\{(x, y) \in X_{0} \times X_{1} \mid u(x)+v(y)=\ell(x, y ; q)\right\}$ is compact and

$$
u(m)=\max _{(x, y) \in S} \ell(m, y ; q)-v(y) \quad \text { for all } m \in X_{0},
$$

where we note that $q$-separation implies the projections of $S \subset M \times M$ onto the first and second copies of $M$ cover $X_{0}$ and $X_{1}$, respectively. Moreover, for fixed $m \in X_{0}$ the supremum is attained at $(x, y)=(m, y) \in S$. Lemma 5.4 implies

$$
s^{q-1} u(m)=\max _{(x, y) \in S, z \in Z_{s}(x, y)} \ell(m, z ; q)+\left(s^{-1}-1\right)^{1-q} \ell(z, y ; q)-s^{q-1} v(y)
$$

and that the maximum is attained at some $(x, y)=(m, y) \in S$ and each $z \in Z_{s}(m, y)$. According to Corollary [5.2, there is a continuous map $W$ : $Z_{s}(S) \longrightarrow S \subset M \times M$ for which $z \in Z_{s}(x, y)$ with $(x, y) \in S$ implies $(x, y)=\left(U_{s}(z), V_{s}(z)\right):=W(z)$. Thus

$$
s^{q-1} u(m)=\max _{z \in Z_{s}(S)} \ell(m, z ; q)+\left(s^{-1}-1\right)^{1-q} \ell\left(z, V_{s}(z) ; q\right)-s^{q-1} v\left(V_{s}(z)\right)
$$

and the maximum is attained at some $z$ satisfying $U_{s}(z)=m$.

We claim $\left(\mu_{0}, \mu_{s}\right)$ is $q$-separated by $\bar{\pi}=\left(U_{s} \times i d\right)_{\#} \mu_{s}$, and

$$
(\bar{u}, \bar{v})=s^{q-1}\left(u, v \circ V_{s}-(1-s)^{1-q} \frac{\ell^{q}}{q} \circ\left(i d \times V_{s}\right)\right) .
$$

Since $z \in Z_{s}(S)$ lies on a geodesic segment whose endpoints $W(z) \in S$ are chronologically separated, $0<s<1$ implies $\left(z, V_{s}(z)\right) \notin \operatorname{sing}(\ell)$; thus $\bar{u} \in C\left(X_{0}\right)$ and $\bar{v} \in C\left(Z_{s}((S))\right)$ inherit continuity from that of $(u, v), V_{s}$ and that of $\ell$ outside $\operatorname{sing}(\ell)$. Since Lemma 2.5 and Proposition 2.9 imply $X_{s} \subset$ $Z_{s}(S)$, compactness of $\bar{S}:=\left\{(x, z) \in \operatorname{spt}\left[\mu_{0} \times \mu_{s}\right] \mid \bar{u}(x)+\bar{v}(z)=\ell(x, z ; q)\right\}$ 
follows from that of $\operatorname{spt}\left[\mu_{0} \times \mu_{s}\right]$ and the upper semicontinuity of $\ell$ shown in Corollary 3.7. Moreover, $\ell \geq 0$ on $\bar{S}$. Our identification of the maximizers in (42) shows spt $\bar{\pi} \subset \bar{S}$, but we must still establish $\ell \neq 0$ on $\bar{S}$.

Given $(x, z) \in \bar{S}$, the identification above asserts $x=U_{s}(z)$. Moreover, $z \in Z_{s}(x, y)$ for $y=V_{s}(z)$. Since Corollary 5.2 also asserts $\pi:=\left(U_{s} \times V_{s}\right)_{\#} \mu_{s}$ maximizes $\ell^{q}$ on $\Pi\left(\mu_{0}, \mu_{1}\right)$, we find $(x, y) \in S$ and furthermore, $\bar{\pi} \in \Pi\left(\mu_{0}, \mu_{s}\right)$. The disjointness of $S$ from $\{\ell \leq 0\}$ guaranteed by $q$-separation implies $y$ is in the chronological future of $x$. Since $z$ lies on the timelike geodesic segment joining $x$ to $y$, this shows $\ell(x, z)>0$ as well. Thus $\bar{S} \subset\{\ell>0\}$ to conclude the proof.

Remark 5.6 (Hopf-Lax / Hamilton-Jacobi semigroup) By symmetry, the potentials which $q$-separate $\mu_{t}$ from $\mu_{1}$ are given by

$$
(\bar{u}, \bar{v})=(1-t)^{q-1}\left(u \circ U_{t}-t^{1-q} \frac{\ell^{q}}{q} \circ\left(U_{t} \times i d\right), v\right) .
$$

instead of (43). Apart from an overall change of sign, $\bar{u}$ should be compared with the Hopf-Lax solution

$$
\tilde{u}(z, t)=\inf _{\substack{\sigma \in C^{1}([0, t] ; M) \\ \sigma(t)=z}}-u(\sigma(0))+\int_{0}^{t} L(\dot{\sigma}(s), \sigma(s) ; q) d s
$$

to the Hamilton-Jacobi semigroup [92]

$$
\frac{\partial \tilde{u}}{\partial t}+H(D \tilde{u} ; q)=0
$$

associated with Hamiltonian $H$ from (23).

Lemma 5.7 (Maps and their Jacobian derivatives) Fix $X, Y \subset M$ compact, $0<q<1$ and $u$ semiconvex and Lipschitz with $u \geq u_{\tilde{q} q}$ in a neighbourhood of $X$.

(i) If $u \oplus u_{\tilde{q}}-\frac{1}{q} \ell^{q} \geq 0$ vanishes at $(\bar{x}, \bar{y}) \in X \times Y$ then $\bar{x} \in$ Dom Du implies $\bar{y}=F_{1}(\bar{x})$ where $F_{s}(x):=\exp _{x} s D H(D u(x), x ; q)$ while $\bar{x} \in$ Dom $\tilde{D}^{2} u$ implies $(\bar{x}, \bar{y}) \notin \operatorname{sing}(\ell)$. Similarly, $\bar{y} \in \operatorname{Dom} D u_{\tilde{q}}$ gives $\bar{x}=\exp _{\bar{y}}-D H\left(-D u_{\tilde{q}}(\bar{y}), \bar{y} ; q\right)$ while $\bar{y} \in \operatorname{Dom} \tilde{D}^{2} u_{\tilde{q}}$ gives $(\bar{x}, \bar{y}) \notin \operatorname{sing}(\ell)$.

(ii) For $\operatorname{vol}_{g}$-a.e. $x \in X$, the approximate derivative $\tilde{D} F_{s}(x): T_{x} M \longrightarrow$ $T_{F_{s}(x)} M$ from Definition 3.8 exists, depends smoothly on $s$, and $\tilde{D} F_{s}(x) w$ gives a Jacobi field along the geodesic $s \in[0,1] \mapsto F_{s}(x)$ for each $w \in T_{x} M$. 
(iii) Moreover,

$$
\left.\frac{\partial}{\partial s}\right|_{s=0} \tilde{D} F_{s}=\left.\tilde{D} \frac{\partial F_{s}}{\partial s}\right|_{s=0}=\left(D^{2} H \circ D u\right) \tilde{D}^{2} u
$$

holds $\mathrm{vol}_{g}$-a.e. on $X$, where the derivatives are computed with respect to the Lorentzian connection, (c.f. (45)), where $H$ is from (23) and we use $\tilde{D}^{2} u$ to denote the approximate Hessian of $u$.

Proof. (i) Observe $u_{\tilde{q} q} \oplus u_{\tilde{q}}-\frac{1}{q} \ell^{q} \geq 0$ holds on $M \times Y$, thus $u \oplus u_{\tilde{q}}-\frac{1}{q} \ell^{q} \geq 0$ on $U \times Y$ where $U$ is the hypothesized neighbourhood of $X$ on which $u$ is Lipschitz and semiconvex. If the latter inequality is saturated at $(\bar{x}, \bar{y}) \in$ $X \times Y$ then $u_{\tilde{q} q} \oplus u_{\tilde{q}}-\frac{1}{q} \ell^{q}$ has zero as a subgradient at $(\bar{x}, \bar{y})$. If $\bar{x} \in \operatorname{Dom} D u$, it follows that $\ell^{q}(\cdot, \bar{y})$ is superdifferentiable at $\bar{x}$ with supergradient $D u(\bar{x})$, whence Corollary 3.7(ii) implies $\bar{y}=\exp _{\bar{x}} D H(D u(\bar{x}), \bar{x} ; q)$ as desired. If, in addition, $\bar{x} \in \operatorname{Dom} \tilde{D}^{2} u$ then the second-order Taylor expansion for $u(x)$ around $\bar{x}$ provides a quadratic upper-bound for $\frac{1}{q} \ell^{q}(x, \bar{y})-u_{\tilde{q}}(\bar{y})$ at $\bar{x}$. This rules out $(\bar{x}, \bar{y}) \in \operatorname{sing}(\ell)$ according to (iv) of the same corollary. Since $u \oplus u_{\tilde{q}}-\frac{1}{q} \ell^{q} \geq 0$ holds on $X \times M$, when equality holds at $(\bar{x}, \bar{y}) \in X \times Y$ with $\bar{y} \in \operatorname{Dom} D u_{\tilde{q}}$ it follows similarly that $\exp _{\bar{y}}^{-1} \bar{x}=-D H\left(-D u_{\tilde{q}}(\bar{y}), \bar{y} ; q\right)$ and — when $\bar{y} \in \operatorname{Dom} \tilde{D}^{2} u_{\tilde{q}}$ - that $(\bar{x}, \bar{y}) \notin \operatorname{sing}(\ell)$.

(ii) For every $\epsilon>0$, semiconvexity implies that outside of a set of volume $\epsilon$ in $U \supset X, u$ agrees with a twice continuously differentiable function $u^{\epsilon} \in$ $C^{2}(M)$; moreover, its approximate second derivative agrees with $D^{2} u^{\epsilon}$ outside of this small set. Thus $F_{s}^{\epsilon}(x):=\exp _{x} s D H\left(D u^{\epsilon}(x), x ; q\right)$ is $C^{1}$ in $x$ and smooth in $s$, and its mixed partial derivatives are continuous and equal: $\frac{\partial}{\partial s} D F_{s}^{\epsilon}=D \frac{\partial}{\partial s} F_{s}^{\epsilon}(x)$ where $D$ denotes derivative with respect to $x$. Given $(w, x(0)) \in T M$, let $r \in[-1,1] \mapsto x(r) \in M$ be a $C^{1}$ curve through $x(0)$ with tangent vector $\dot{x}(0)=w$. Then $r \in[-1,1] \mapsto F_{s}^{\epsilon}(x(r))$ is a $C^{1}$ geodesic variation since $s \in[0,1] \mapsto F_{s}^{\epsilon}(x(r))$ is a geodesic for each $r \in[-1,1]$. Thus $\left.\frac{\partial}{\partial r}\right|_{r=0} F_{s}^{\epsilon}(x(r))=D F_{s}^{\epsilon}(x(0)) w$ is a Jacobi field (by e.g. Lemma 8.3 of [77]). Since the approximate derivative $\tilde{D} F_{s}(x)$ agrees with $D F_{s}^{\epsilon}(x)$ outside of a set of volume $\epsilon$, and $\epsilon>0$ is arbitrary, we find $\tilde{D} F_{s}(x(0)) w$ to depend smoothly on $s$ and be a Jacobi field for $x(0) \in U$ in a subset of full volume.

(iii) Differentiating the vector field $\left.\frac{\partial F_{s}^{\epsilon}(x)}{\partial s}\right|_{s=0}=D H\left(D u^{\epsilon}(x), x ; q\right)$ using the Lorentzian connection yields

$$
\left.D_{k} \frac{\partial}{\partial s}\right|_{s=0} F_{s}^{\epsilon}(x)^{i}=H^{i j} u_{j k}^{\epsilon}
$$


since $H(p, x ; q)=-|p|_{g}^{q^{\prime}} / q^{\prime}$ with $\frac{1}{q}+\frac{1}{q^{\prime}}=1$ whenever $p=D u$ is past-directed and timelike. We may interchange the order of $x$ and $s$ derivatives as in (ii). Since these derivatives of $F^{\epsilon}$ and $u^{\epsilon}$ agree with the corresponding approximate derivatives of $F$ and $u$ outside a set of volume $\epsilon>0$, we obtain (44).

We are now in a position to characterize the joint measure $\pi \in \Pi(\mu, \nu)$ maximizing (6). Let $\mathcal{P}_{c}^{a c}(M) \subset \mathcal{P}_{c}(M)$ denote the measures $\mu$ which are absolutely continuous with respect to the Lorentzian volume $\operatorname{vol}_{g}$.

Theorem 5.8 (Characterizing optimal maps) Fix $0<q<1$. If $(\mu, \nu) \in$ $\mathcal{P}_{c}(M)^{2}$ is q-separated by $(\pi, u, v)$, and $\mu \in \mathcal{P}_{c}^{a c}(M)$, setting $X \times Y:=$ $\operatorname{spt}[\mu \times \nu]$ implies (i) there is a unique map $F(x)=\exp _{x} D H(D \bar{u}(x), x ; q)$ with $\nu=F_{\#} \mu$ such that $\bar{u}$ is Lipschitz and satisfies

$$
\bar{u}(x)=\max _{y \in Y} \frac{1}{q} \ell^{q}(x, y)-\bar{u}_{\tilde{q}}(y)
$$

on a neighbourhood of $X$; in this case $\pi=(i d \times F)_{\#} \mu$ uniquely maximizes (6), $u$ is semiconvex in a neighbourhood of $X$, and both $D u=D \bar{u}$ and $(x, F(x)) \notin$ $\operatorname{sing}(\ell)$ hold $\mu$-a.e. (ii) If, in addition, $\nu \in \mathcal{P}_{c}^{a c}(M)$ then $F \circ G(y)=y$ holds $\mu$ a.e. and $G(F(x))=x$ holds $\nu$-a.e. where $G(y):=\exp _{y}-D H\left(-D u_{\tilde{q}}(y), y ; q\right)$. Here $H$ is from (23) and $F_{\#}$ from Definition 2.2.

Proof. (i) Theorem 4.3 shows $(u, v)=\left(v_{q}, u_{\tilde{q}}\right)$ on $X \times Y$, and that $u:=v_{q}$ and $v:=u_{\tilde{q}}$ are semiconvex Lipschitz functions on neighbourhoods of $X$ and $Y$. It also shows $(u, v)$ attains the infimum (33) and $\pi$ attains the maximum (6). Let $S \subset X \times Y$ be the zero set of the non-negative function $u \oplus v-\frac{1}{q} \ell^{q}$. When $q<1$, for each $(x, y) \in S$ with $x \in \operatorname{Dom} \tilde{D}^{2} u$, Lemma 5.7 goes on to assert $y=F_{1}(x):=\exp _{x} D H(D u(x), x ; q)$ and $\left(x, F_{1}(x)\right) \notin \operatorname{sing}(\ell)$. Since Dom $\tilde{D}^{2} u$ is a set of full $\operatorname{vol}_{g}$ (hence $\mu \ll \operatorname{vol}_{g}$ ) measure by Alexandrov's theorem (e.g. [17]), we deduce $\pi=\left(i d \times F_{1}\right)_{\#} \mu$ from e.g. Lemma 3 of [2]. If $\pi^{\prime} \in \Pi(\mu, \nu)$ also maximizes (6), then $\pi^{\prime}$ vanishes outside $S$ because of the duality (33), and we conclude $\pi^{\prime}=\left(i d \times F_{1}\right)_{\#} \mu$ as above. This shows uniqueness of the maximizer when $q<1$ and $\mu \in \mathcal{P}_{c}^{a c}(M)$.

Now suppose $F_{\#} \mu=\nu$, where $F$ is defined as in the statement of the theorem and $\bar{u}$ is Lipschitz, semiconvex and satisfies (46) in a neighbourhood of $X$. We claim $\bar{\pi}=(i d \times F)_{\#} \mu$ maximizes (33). For each $x \in X \cap \operatorname{Dom} D \bar{u}$, the point $y \in Y$ attaining the maximum (46) is given by $y=F(x)$, according to Lemma 5.7. Thus

$$
\frac{1}{q} \ell^{q}(x, F(x))=\bar{u}(x)+\bar{u}_{\tilde{q}}(F(x))
$$


holds on a set $X \cap \operatorname{Dom} D \bar{u}$ whose complement is $\mu$-negligible. Integrating this identity against $\mu$ yields

$$
\frac{1}{q} \int_{M \times M} \ell^{q}(x, y) d \bar{\pi}(x, y)=\int_{M} \bar{u}(x) d \mu(x)+\int_{M} \bar{u}_{\tilde{q}}(y) d \nu(y) .
$$

where $F_{\#} \mu=\nu$ has been used. This shows $\bar{\pi}$ maximizes (6), in view of the duality (33). The uniqueness of maximizer established above implies $(i d \times F)_{\#} \mu=\left(i d \times F_{1}\right)_{\#} \mu$, from which we conclude $F=F_{1}$ holds $\mu$-a.e. Finally, $D u(\bar{x})=D \bar{u}(\bar{x})$ on the set $X \cap \operatorname{Dom} D u \cap \operatorname{Dom} D \bar{u}$ of full $\mu$-measure: Theorem 3.6 and its corollary show there cannot be multiple action minimizing geodesics joining $\bar{x}$ to $F(\bar{x})$ unless $x \in M \mapsto \ell^{q}(x, F(\bar{x}))$ is subdifferentiable but not superdifferentiable at $x=\bar{x}$, which would contradict the vanishing of $u(x)+u_{\tilde{q}}(F(\bar{x}))-\frac{1}{q} \ell^{q}(x, F(\bar{x})) \geq 0$ at $x=\bar{x} \in \operatorname{Dom} D u$.

(ii) When $\nu \in \mathcal{P}_{c}^{a c}(M)$ a similar argument (or symmetry) shows $\pi=$ $(G \times i d)_{\#} \nu$. In particular, the set $(X \cap \operatorname{Dom} D u) \times(Y \cap \operatorname{Dom} D v)$ is full measure for $\pi$, and for each point $(x, y)$ in this set we have $y=F(x)$ and $x=G(y)$. This shows $G$ acts $\mu$-a.e as left-inverse to $F$, and $\nu$-a.e. as rightinverse to $F$.

Corollary 5.9 (Lagrangian characterization of q-geodesics) Fix $0<$ $q<1$. If $\left(\mu_{0}, \mu_{1}\right) \in \mathcal{P}_{c}(M)^{2}$ is q-separated by $(\pi, u, v)$ and $\mu_{0} \in \mathcal{P}_{c}^{a c}(M)$ then $F_{s}(x):=\exp _{x} s D H(D u(x), x ; q)$ defines the unique q-geodesic $s \in[0,1] \mapsto$ $\mu_{s}:=F_{s \#} \mu_{0}$ in $\mathcal{P}(M)$ linking $\mu_{0}$ to $\mu_{1}$. (We assume $u$ has been extended to a neighbourhood of $X$ by setting $u:=v_{q}$ in (34), where $X \times Y:=\operatorname{spt}\left[\mu_{0} \times \mu_{1}\right]$.) Moreover, $\mu_{s} \in \mathcal{P}_{c}^{a c}(M)$ if $s<1$.

Proof. Under these hypotheses, Theorem 5.8(i)-(ii) assert the maximum (6) to be uniquely attained by $\pi=\left(i d \times F_{1}\right)_{\#} \mu$, where $\pi[\operatorname{sing}(\ell)]=0$. Theorem 2.11 then implies the unique $q$-geodesic $\mu_{s}$ joining $\mu_{0}$ to $\mu_{1}$ to be given by $z_{s \#} \pi=F_{s \#} \mu$, where the last identification follows from $z_{s}\left(x, F_{1}(x)\right)=F_{s}(x)$.

For $s<1$, Theorem 5.3 asserts $F_{s}$ has a Lipschitz inverse. Thus $F_{s}^{-1}(V)$ has zero Lorentzian volume if $V \subset M$ does, in which case absolute continuity of $\mu_{0}$ implies $\mu_{s}(V)=\mu_{0}\left(F_{s}^{-1}(V)\right)$ also vanishes, establishing absolute continuity of $\mu_{s}$. Compactness of its support is asserted by Corollary 2.10.

For reference, let us also state the Lorentzian analog of Theorem 11.1 of [92]; its omitted proof combines Theorem 3.83 of [6] with Lemma 5.5.3 of [9] applied in local coordinates, as in the Riemannian case. 
Theorem 5.10 (Jacobian equation) Let $\left(M^{n}, g\right)$ be a Lorentzian manifold with a compatible Riemannian metric $\tilde{g}$. Let $0 \leq f \in L^{1}\left(M, d \mathrm{vol}_{g}\right)$ and let $F: M \longrightarrow M$ be Borel. Define $d \mu(x)=f(x) d \operatorname{vol}_{g}(x)$ and $\nu:=F_{\#} \mu$. Assume that: (i) $f$ vanishes outside a measurable set $\Sigma \subset M$ on which $F$ is injective; and (ii) $F$ is approximately differentiable almost everywhere on $\Sigma$.

Define $J F(x):=|\operatorname{det} \tilde{D} F(x)|$ a.e. on $\Sigma$, where $\tilde{D} F$ denotes the approximate gradient of $F$. Then $\nu \ll \operatorname{vol}_{g}$ if and only if $J F(x)>0$ a.e. In that case $\nu$ vanishes outside $F(\Sigma)$, and its density $\rho$ is determined by the equation

$$
f(x)=\rho(F(x)) J F(x) .
$$

Corollary 5.11 (Monge-Ampère type equation) Under the hypotheses of Theorem 5.8(i)-(ii), F is countably Lipschitz and the Jacobian equation

$$
\rho_{0}(x)=\rho_{1}(F(x)) J F(x)
$$

holds $\rho_{0}$-a.e., where $\rho_{0}=d \mu / d \operatorname{vol}_{g}, \rho_{1}=d \nu / d \operatorname{vol}_{g}$ and $J F(x)=|\operatorname{det} \tilde{D} F(x)|$, with $\tilde{D} F$ denoting the approximate derivative of $F$ from Definition 3.8 .

Proof. The potential $u=u_{\tilde{q} q}$ of Theorem 5.8 is semiconvex by Theorem 4.3. As a consequence $u$ agrees with a $C^{2}$ function outside of a set of arbitrarily small volume. Thus $F$ is countably Lipschitz, hence approximately differentiable $\operatorname{vol}_{g}$-a.e. It is also injective $\mu$-a.e., according to Theorem 5.8(iii). The Jacobian equation (48) now follows from Theorem 5.10.

We call (48) a Monge-Ampère type equation since it reduces to a secondorder degenerate elliptic equation for the $\frac{1}{q} \ell^{q}$-convex potential $u$ of Theorem 5.8, as in e.g. 92. Combining Corollaries 5.9 and 5.11 yields an analogous equation (39) for the density $\rho_{s}:=d \mu_{s} / d \mathrm{vol}_{g}$ along the $q$-geodesic $s \in[0,1] \mapsto \mu_{s} \in \mathcal{P}_{c}^{a c}(M)$. This equation holds $\mu_{0}$-a.e., though the set where it holds may depend on $s \in[0,1]$.

\section{Entropic convexity from Ricci lower bounds}

The key to understanding the behaviour of entropy along $q$-geodesics $s \in$ $[0,1] \mapsto F_{s \#} \mu_{0} \in \mathcal{P}_{c}^{a c}(M)$ is to analyze the Jacobian factors $J F_{s}(x):=$ $\left|\operatorname{det} \tilde{D} F_{s}(x)\right|$ which appear in the Monge-Ampère type equations (39). In a moving frame along the proper time maximizing segment $s \in[0,1] \mapsto F_{s}(x)$, Lemma 5.7 asserts $A_{s}(x):=\tilde{D} F_{s}(x)$ is a matrix of Jacobi fields. The present 
section begins with a proposition harvesting consequences of the fact that its logarithmic derivative $B_{s}(x):=A_{s}^{\prime}(x) A_{s}(x)^{-1}$ in time satisfies a matrix Riccati equation, whose trace involves the Ricci curvature in the direction of the worldline $s \in[0,1] \mapsto F_{s}(x)$; c.f. [37] [32] [89] and Raychaudhuri's equation. After a technical lemma, Theorem 6.4 gives explicit expressions for the first two derivatives of the Boltzmann-Shannon and relative entropies $E_{V}\left(F_{s \#} \mu_{0}\right)$ along the geodesic in question. Its corollary translates a non-negative lower Ricci curvature bound into quantified convexity of the Boltzmann-Shannon entropy along $q$-geodesics.

Proposition 6.1 (Jacobian along $q$-geodesics) Fix $0<q<1$ and let $\left(\mu_{0}, \mu_{1}\right) \in \mathcal{P}_{c}^{a c}(M)^{2}$ be q-separated by $(\pi, u, v)$. Set $X \times Y=\operatorname{spt}\left[\mu_{0} \times \mu_{1}\right]$, $u:=v_{q}$ and $F_{s}(x):=\exp _{x} s D H(D u(x), x ; q)$. For $\operatorname{vol}_{g}$-a.e. $x \in X$, the approximate derivative $A_{s}(x):=\tilde{D} F_{s}(x): T_{x} M \longrightarrow T_{F_{s}(x)} M$ exists, is invertible, depends smoothly on $s \in[0,1]$, and $\phi(s):=-\log \left|\operatorname{det} A_{s}(x)\right|$ satisfies

$$
\begin{aligned}
\phi^{\prime}(s) & =-\operatorname{Tr} B_{s}(x), \\
\phi^{\prime \prime}(s) & =\operatorname{Ric}_{F_{s}(x)}\left(F_{s}^{\prime}(x), F_{s}^{\prime}(x)\right)+\operatorname{Tr}\left[B_{s}^{2}(t)\right], \\
\text { and } \operatorname{Tr}\left[B_{s}^{2}(x)\right] & \geq \frac{1}{n}\left(\operatorname{Tr} B_{s}(x)\right)^{2},
\end{aligned}
$$

where $B_{s}(x):=A_{s}^{\prime}(x) A_{s}(x)^{-1}$ and ${ }^{\prime}:=\frac{\partial}{\partial s}$ and the Ricci curvature Ric is computed with respect to the Lorentzian connection.

Proof. For vol $\operatorname{vo}$-a.e. $x \in X$, Lemma 5.7 asserts that $A_{s}(x)$ and $\tilde{D}^{2} u(x)$ exist, and that $s \in[0,1] \mapsto A_{s}(x) w$ is a (smooth) Jacobi field for each $w \in T_{x} M$, with $B_{0}(x)=D^{2} H(D u(x)) \tilde{D}^{2} u(x)$, in view of Theorem 4.3(iv). Corollorary 5.11 asserts $A_{1}(x)$ is invertible a.e. Fixing such an $x \in X$, since $A_{0}(x)=I$ the set of $s$ values for which $\operatorname{det} A_{s}(x)=0$ forms a closed subset of $(0,1)$ which we shall presently show to be empty. Outside of this set, from $\phi(s)=-\operatorname{Tr} \log \left|A_{s}(x)\right|$ we compute

$$
\begin{aligned}
\phi^{\prime}(s) & =-\operatorname{Tr} B_{s}(x), \\
\text { and } \quad \phi^{\prime \prime}(s) & =-\operatorname{Tr}\left[A_{s}^{\prime \prime}(x) A_{s}(x)^{-1}\right]+\operatorname{Tr}\left[B_{s}^{2}(x)\right] .
\end{aligned}
$$

Since $s \in[0,1] \mapsto A(s) w \in T_{F_{s}(x)} M$ is a Jacobi field for each $w \in T_{x} M$, we can evaluate $\operatorname{Tr} \bar{A}^{\prime \prime}(s) \bar{A}(s)^{-1}$ via Jacobi's equation:

$$
\begin{aligned}
0 & =\left(\nabla_{F^{\prime}}\left(\nabla_{F^{\prime}} A_{\bar{j}}^{i}\right)+R_{j k l}^{i} F^{\prime j} F^{\prime l} A_{\bar{j}}^{k}\right)\left(A^{-1}\right)_{i}^{\bar{j}} \\
& =\operatorname{Tr} A^{\prime \prime}(s) A(s)^{-1}+\operatorname{Ric}\left(F^{\prime}, F^{\prime}\right)
\end{aligned}
$$


to arrive at (50); here barred and unbarred indices refer to coordinate systems at $x$ and $F_{s}(x)$ respectively.

We can now prove (51), at least when $s=0$. Indeed, this follows from Cauchy-Schwartz inequality for the Hilbert-Schmidt norm $\|C\|^{2}:=\operatorname{Tr} C^{*} C$ on $n \times n$ matrices $C$, which asserts

$$
\left(\operatorname{Tr} C^{*} D\right)^{2} \leq\left(\operatorname{Tr} C^{*} C\right)\left(\operatorname{Tr} D^{*} D\right),
$$

when applied to $C=\sqrt{D^{2} H} D^{2} u \sqrt{D^{2} H}$ and $D=I$, noting $\operatorname{Tr} D^{*} D=n$, $\operatorname{Tr} C^{*}=\operatorname{Tr} C=\operatorname{Tr} B_{0}$ and $\operatorname{Tr} C^{*} C=\operatorname{Tr} C^{2}=\operatorname{Tr} B_{0}^{2}$. Here convexity of $H(p)$ plays the crucial role of ensuring $D^{2} H$ is non-negative definite, hence admits a matrix square-root.

The next step in the proof is to propagate the estimate (51) from $s=0$ to $s>0$ using the (Hopf-Lax) semigroup property for $q$-geodesics. Theorem 5.3 asserts that $F_{s}^{-1}$ extends to a Lipschitz map on spt $\mu_{s}$, whose image must have full measure in spt $\mu_{0}$ since $\left(F_{s}^{-1}\right)_{\#} \mu_{s}=\mu_{0}$. Defining $F_{s}^{t}:=F_{s} \circ F_{t}^{-1}$ whenever $t \leq s$, we deduce $s \in[0,1] \mapsto \mu_{s}=\left(F_{s}^{t}\right)_{\#} \mu_{t}$ is the $q$-geodesic connecting $\mu_{t}$ to $\mu_{1}$. Moreover, $F_{s}^{t}$ can be confirmed to be the $\ell^{q}$-optimal map between $\mu_{t}$ and $\mu_{s}$ as a consequence of Proposition 2.9 and Theorem 2.11. For fixed $t$ and $\mu_{t}$-a.e. $z$ set $\bar{A}_{s}(z)=D F_{s}^{t}(z)$ and $\bar{B}_{s}(z)=\bar{A}_{s}^{\prime}(z) A_{s}(z)^{-1}$. The preceding paragraph yields

$$
\operatorname{Tr}\left[\bar{B}_{t}^{2}(z)\right] \geq \frac{1}{n}\left(\operatorname{Tr} \bar{B}_{t}(z)\right)^{2}
$$

But $D F_{s}^{t}=D F_{s} \circ D F_{t}^{-1}$ and $\left(D F_{s}^{t}\right)^{\prime}=D F_{s}^{\prime} \circ D F_{t}^{-1}$, whence $\bar{B}_{s}(z)=$ $\left(D F_{s}^{t}(x)\right)^{\prime}\left(D F_{s}^{t}(z)\right)^{-1}=B_{s}\left(F_{t}^{-1}(z)\right)$. Thus (52) translates into the desired bound (51), at least on a set $X_{s}$ of full $\mu_{0}$ measure. Although $X_{s}$ here depends on $s=t \in[0,1]$, the bound (51) holds on the intersection $\cap_{s \in \mathbf{Q} \cap[0,1]} X_{s}$ for all rational $s$, hence for all $s \in[0,1]$ since $B_{s}(x)$ depends smoothly on $s$.

Finally, (49)-(51) combine with $\left|F_{s}^{\prime}(x)\right|=\ell\left(x, F_{1}(x)\right)$ to show

$$
\phi^{\prime \prime}(s)-\frac{1}{n}\left(\phi^{\prime}(s)\right)^{2} \geq K \ell^{2}\left(x, F_{1}(x)\right)
$$

where the constant $K$ is a lower bound for the Ricci curvature of $M$ on the compact set $Z\left(\operatorname{spt}\left[\mu_{0} \times \mu_{1}\right]\right)$ of Lemma 2.5. In particular, $\phi(s)$ is semiconvex on the open set $S(x):=\{s \mid \phi(s) \neq-\infty\}$. This yields a lower bound for $\phi(s)$ throughout $[0,1]$ in terms of $\phi(0)$ and $\phi^{\prime}(0)$ (or of $\left(\phi, \phi^{\prime}\right)(\epsilon)$ if $\left.\phi^{\prime}(0)=-\infty\right)$, which shows $S(x)$ to be empty and $A_{s}(x)$ to be invertible. 
Remark 6.2 (Relevance of Lipschitz inverse maps) The Monge-Mather shortening estimate of Theorem 5.3 is essential only to extend (51) from $s=0$ to $s>0$. Once we have this extension, one can deduce the absolute continuity of $F_{s \#} \mu_{0}$ for $s \in(0,1)$ from Theorem 5.10 using the positivity of $J F_{s}(x)$ provided by Proposition [6.1, as an alternative to Corollary 5.9.

Lemma 6.3 (Second finite-difference representation) If $\phi \in L^{\infty}([0,1])$ is semiconvex on $(0,1)$ and $g(s, t):=\min \{s, t\}-$ st, then

$$
(1-t) \phi(0)+t \phi(1)-\phi(t)=\int_{[0,1]} \phi^{\prime \prime}(s) g(s, t) d s
$$

for each $t \in[0,1]$, where $\phi^{\prime \prime}$ denotes the distributional second derivative of $\phi$.

Proof. Semiconvexity and boundedness implies $\phi$ has a continuous extension $\bar{\phi}$ to $[0,1]$, which coincides with $\phi$ except perhaps at the endpoints. For $\bar{\phi}$, the representation (53) is asserted by Villani in (16.5) of [92. When $\phi$ differs from $\bar{\phi}$, then $\phi^{\prime \prime}$ differs from $\bar{\phi}^{\prime \prime}$ only by derivatives of Dirac distributions at the endpoints:

$$
\phi^{\prime \prime}(s)-\bar{\phi}^{\prime \prime}(s)=-(\phi(0)-\bar{\phi}(0)) \delta^{\prime}(s)+(\phi(1)-\bar{\phi}(1)) \delta^{\prime}(s-1) .
$$

It is not hard to verify the representation (53) extends from $\bar{\phi}$ to $\phi$, after noting for each $t \in[0,1]$ that $g(s, t)$ depends smoothly on $s$ in a neighborhood of the endpoints of $[0,1]$, where it vanishes. (We can extend $\phi$ and $\bar{\phi}$ to be locally constant outside $(0,1)$ and $g(s, t)$ to be compactly supported and smooth outside $s=t \in[0,1]$ to facilitate this calculation.)

Theorem 6.4 (Displacement Hessian of relative entropy) Fix $0<q<$ 1 and $V \in C^{2}(M)$ on a globally hyperbolic spacetime. Let $s \in[0,1] \mapsto \mu_{s}=$ $\left(F_{s}\right)_{\#} \mu_{0} \in \mathcal{P}_{c}^{a c}(M)$ be one of the q-geodesics described by Corollary 5.9. If $e(0)$ and $e(1)$ are finite, then: (a) the relative entropy $e(s):=E_{V}\left(\mu_{s}\right)$ of (9) is continuous and semiconvex on $s \in[0,1]$ and continuously differentiable on $s \in(0,1)$, with

$$
\begin{aligned}
e^{\prime}(s) & =\int_{M}\left[D V_{F_{s}(x)} F_{s}^{\prime}(x)-\operatorname{Tr} B_{s}(x)\right] d \mu_{0}(x) \quad \text { and } \\
e^{\prime \prime}(s) & =\int_{M}\left[\operatorname{Tr}\left(B_{s}^{2}(x)\right)+\left(\operatorname{Ric}+D^{2} V\right)_{F_{s}(x)}\left(F_{s}^{\prime}(x), F_{s}^{\prime}(x)\right)\right] d \mu_{0}(x)
\end{aligned}
$$


holding on $[0,1]$ in the distributional sense. Here $A_{s}(x):=\tilde{D} F_{s}(x): T_{x} M \longrightarrow$ $T_{F_{s}(x)} M$ denotes the approximate derivative of $F_{s}, B_{s}(x):=A_{s}^{\prime}(x) A_{s}(x)^{-1}$, $':=\frac{\partial}{\partial s}$ and $\operatorname{Tr}\left[B_{s}(x)^{2}\right] \geq \frac{1}{n}\left(\operatorname{Tr} B_{s}(x)\right)^{2}$. (b) The integral expression (55) for $e^{\prime \prime}(s)$ depends lower semicontinuously on $s \in[0,1]$; the integrand is bounded below.

Proof. Our strategy will be to produce a finite second difference representation of $e$ using Lemma 6.3.

Let $F_{s}(x):=\exp _{x} s D H(D u(x), x ; q)$ and $\mu_{s}=\left(F_{s}\right)_{\#} \mu_{0} \in \mathcal{P}_{c}^{a c}(M)$ be from Corollary 5.9, Proposition 6.1 asserts that $J F_{s}(x):=\left|\operatorname{det} \tilde{D} F_{s}(x)\right|$ exists and depends smoothly on $s \in[0,1]$ for each $x$ in a subset $X_{0}$ of full measure in spt $\mu_{0}$. Letting $\rho_{s}:=d \mu_{s} / d \operatorname{vol}_{g}$, Corollary [5.11 gives

$$
\rho_{s}\left(F_{s}(x)\right) J F_{s}(x)=\rho_{0}(x)>0
$$

on a subset $X_{s} \subset X_{0}$ of full $\mu_{0}$ measure.

Letting $Z:=Z\left(\operatorname{spt}\left[\mu_{0} \times \mu_{1}\right]\right)$ denote the compact set from Lemma 2.5, since spt $\mu_{s} \subset Z$ the (Borel) change of variables $y=F_{s}(x)$ and (10) yield

$$
\begin{aligned}
-\infty & <-\log \int_{Z} e^{-V} d \operatorname{vol}_{g} \\
& \leq e(s) \\
& =\int_{M}\left[\log \rho_{s}(y)+V(y)\right] d \mu_{s}(y) \\
& =\int_{M}\left[\log \rho_{s}\left(F_{s}(x)\right)+V\left(F_{s}(x)\right)\right] d \mu_{0}(x) \\
& =\int_{M}\left[\log \rho_{0}(x)-\log \left|J F_{s}(x)\right|+V\left(F_{s}(x)\right)\right] d \mu_{0}(x)
\end{aligned}
$$

where the last identity follows from (56). Thus

$$
(1-t) e(0)+t e(1)-e(t)=\int_{M}\left[(1-t) \phi_{x}(0)+t \phi_{x}(1)-\phi_{x}(t)\right] d \mu_{0}(x) .
$$

where

$$
\phi_{x}(s)=-\log \left|J F_{s}(x)\right|+V\left(F_{s}(x)\right)
$$

For $x \in X_{0}$ (which forms a set of full $\mu_{0}$ measure), setting $A_{s}(x)=$ $\tilde{D} F_{s}(x)$ and $B_{s}(x)=A_{x}^{\prime}(x) A_{s}(x)^{-1}$, Proposition 6.1 yields $\operatorname{Tr}\left[B_{s}(x)^{2}\right] \geq$ 


$$
\begin{aligned}
\frac{1}{n}\left(\operatorname{Tr} B_{s}(x)\right)^{2} \geq 0, & \\
\phi_{x}^{\prime}(s) & =D V\left(F_{s}(x)\right) F_{s}^{\prime}(x)-\operatorname{Tr} B_{s}(x) \quad \text { and } \\
\phi_{x}^{\prime \prime}(s) & =\operatorname{Tr}\left[B_{s}(x)^{2}\right]+\left(\operatorname{Ric}+D^{2} V\right)\left(F_{s}^{\prime}(x), F_{s}^{\prime}(x)\right) \\
& \geq K_{Z} \ell\left(x, F_{1}(x)\right)^{2}
\end{aligned}
$$

where $F_{s}^{\prime}=\frac{\partial F_{s}}{\partial s} \in T_{F_{s}(x)} M$ and $\nabla_{F_{s}^{\prime}} F_{s}^{\prime}=0$ since $s \in[0,1] \mapsto F_{s}(x)$ is an action minimizing geodesic segment. Here $K_{Z}$ denotes a lower bound for Ric $+D^{2} V \geq K_{Z} g$ on the compact set $Z \supset$ spt $\mu_{s}$ defined above, and we have used geodesy to conclude $\left|F_{s}^{\prime}(x)\right|=\ell\left(x, F_{1}(x)\right)$.

Applying Lemma 6.3 to (58) yields

$$
\begin{aligned}
& (1-t) e(0)+t e(1)-e(t) \\
= & \int_{M} \int_{[0,1]} \phi_{x}^{\prime \prime}(s) g(s, t) d s d \mu_{0}(x) \\
= & \int_{M} \int_{[0,1]}\left[\operatorname{Tr}\left(B_{s}^{2}(x)\right)+\left(\operatorname{Ric}+D^{2} V\right)\left(F_{s}^{\prime}(x), F_{s}^{\prime}(x)\right)\right] g(s, t) d s d \mu_{0}(x)(63) \\
\geq & \frac{K_{Z}}{2} t(1-t) \int_{M} \ell\left(x, F_{1}(x)\right)^{2} d \mu_{0}(x)
\end{aligned}
$$

Since each subsegment of a $q$-geodesic is a $q$-geodesic, we deduce

$$
\begin{aligned}
\frac{e(s)+e(t)}{2}-e\left(\frac{s+t}{2}\right) & \geq \frac{K_{Z}}{8} \int_{M} \ell\left(F_{s}(x), F_{t}(x)\right)^{2} d \mu_{0}(x) \\
& =\frac{K_{Z}}{8} \int_{M} \ell\left(x, F_{1}(x)\right)^{2} d \mu_{0}(x) \\
& \geq-\frac{1}{8} \min \left\{K_{Z}, 0\right\} \sup _{x, y \in Z} \ell(x, y)^{2} \\
& >-\infty
\end{aligned}
$$

for all $0 \leq s \leq t \leq 1$. This shows the semiconvexity and upper boundedness of $e$ on $[0,1]$, and continuity on $(0,1)$, since (57) bounds $e(s)$ below and we have assumed finiteness of $e(0)$ and $e(1)$.

Applying Lemma 6.3 to $e$, (63) now yields

$$
\begin{aligned}
e^{\prime \prime}(s) & =\int_{M}\left[\operatorname{Tr}\left(B_{s}^{2}(x)\right)+\operatorname{Ric}\left(F_{s}^{\prime}(x), F_{s}^{\prime}(x)\right)\right] d \mu_{0}(x) \\
& \geq K_{Z} \int_{M} \ell\left(x, F_{1}(x)\right)^{2} d \mu_{0}(x)
\end{aligned}
$$


in the distributional sense. The lower bound (65) implies continuity of $e$ at the endpoints of $[0,1]$, since otherwise $e^{\prime \prime}$ would contain a derivative of a Dirac delta measure. Using (59)-(61) and Fubini's theorem, we can also integrate (55) twice to obtain

$$
\begin{aligned}
e^{\prime}(s) & =c_{1}+\int_{M}\left[D V_{F_{s}(x)} F_{s}^{\prime}(x)-\operatorname{Tr} B_{s}(x)\right] d \mu_{0}(x) \quad \text { and } \\
e(s) & =c_{0}+c_{1} s+\int_{M}\left[V\left(F_{s}(x)\right)-\log \left|J F_{s}(x)\right|\right] d \mu_{0}(x) .
\end{aligned}
$$

The boundary values determine the constants $c_{0}=E_{0}\left(\mu_{0}\right)$ and $c_{1}=0$ of integration by comparison with (57), to establish (54).

On a set $X_{0}$ of full measure, the integrand $\phi_{x}^{\prime \prime}(s)$ depends smoothly on $s \in[0,1]$ and can be bounded below independently of $x \in Z$ using (61). Lower semicontinuity of the integral (55) representing $e^{\prime \prime}(s)$ therefore follows from Fatou's lemma. Similarly, the addition of a linear term $k s$ makes the integrand $\phi_{x}^{\prime}(s)$ from (60) increase continuously in $s \in[0,1]$; continuity of $e^{\prime}(s)$ on $(0,1)$ then follows from the representation (54) by Lebesgue's dominated convergence theorem, to conclude the proof.

Definition 6.5 ((K,N) convexity; c.f. 39]) Fix $K \in \mathbf{R}$ and $N>0$. A function $e:[0,1] \longrightarrow[-\infty, \infty]$ is said to be $(K, N)$-convex if $e$ is upper semicontinuous, Dom $e:=\{s \in[0,1] \mid e(s)<\infty\}$ is connected, and either $e^{-1}(-\infty)$ contains the interior I of Dom $e$ or is empty, and in the latter case: $e$ is semiconvex throughout I and satisfies

$$
e^{\prime \prime}(s)-\frac{1}{N}\left(e^{\prime}(s)\right)^{2} \geq K
$$

there, in the distributional sense. The last clause merely means the second derivative of e is interpreted distributionally; semiconvexity implies $e^{\prime}(s)$ has no singular part, hence $e^{\prime}(s)^{2}$ can be interpreted in the pointwise a.e. sense.

Given a globally hyperbolic spacetime $\left(M^{n}, g\right)$ and $0<q \leq 1$, a functional $E: \mathcal{P}(M) \longrightarrow \mathbf{R} \cup\{ \pm \infty\}$ is said to be weakly $(K, N, q)$-convex for $Q \subset$ $\mathcal{P}(M)^{2}$ if for each $\left(\mu_{0}, \mu_{1}\right) \in Q$ there is a q-geodesic in $\mathcal{P}(M)$ joining $\mu_{0}$ to $\mu_{1}$ on which $E\left(\mu_{s}\right)$ is $\left(K \ell_{q}\left(\mu_{0}, \mu_{1}\right)^{2}, N\right)$-convex. E is said to be $(K, N, q)$-convex for $Q$ if, in addition, $E\left(\mu_{s}\right)$ is $\left(K \ell_{q}\left(\mu_{0}, \mu_{1}\right)^{2}, N\right)$-convex for all q-geodesics $s \in[0,1] \mapsto \mu_{s} \in \mathcal{P}(M)$ with endpoints in $Q$. 
Recall also the definition (11) of the $N$-Bakry-Émery-Ricci tensor

$$
\operatorname{Ric}_{a b}^{(N, V)}:=\operatorname{Ric}_{a b}+\nabla_{a} \nabla_{b} V-\frac{1}{N-n} \nabla_{a} V \nabla_{b} V
$$

Corollary 6.6 (Entropic convexity from timelike lower Ricci bounds) Let $\left(M^{n}, g\right)$ be a globally hyperbolic spacetime. Fix $V \in C^{2}(M)$ and $N>n$. If $\operatorname{Ric}^{(N, V)}(v, v) \geq K|v|_{g}^{2} \geq 0$ holds in every timelike direction $(v, x) \in T M$, then for each $0<q<1$ the relative entropy $E_{V}(\mu)$ of (9) is $(K, N, q)$-convex for the set $Q \subset \mathcal{P}_{c}^{a c}(M)^{2}$ of probability measures with q-separated endpoints.

Proof. Fix $0<q<1$. If $\left(\mu_{0}, \mu_{1}\right) \in Q$ then Corollary 5.9 describes the unique $q$-geodesic $s \in[0,1] \mapsto \mu_{s} \in \mathcal{P}(M)$ joining any such pair of $q$-separated endpoints, and asserts that $\mu_{s} \in \mathcal{P}_{c}^{a c}(M)$ for each $s \in[0,1]$. Moreover, $e(s):=E_{V}\left(\mu_{s}\right)>-\infty$ by (10). If $e(s)$ is finite at $s=0$ and $s=1$, Jensen's inequality combines with Theorem 6.4 to estimate

$$
\begin{aligned}
\frac{1}{N} e^{\prime}(s)^{2} & \leq \int_{M}\left(\frac{1+\epsilon^{-1}}{N}\left|D V\left(F_{s}\right) \cdot F_{s}^{\prime}\right|^{2}+(1+\epsilon) \frac{n}{N} \operatorname{Tr}\left[B_{s}^{2}\right]\right) d \mu_{0} \\
& =\int_{M}\left(\frac{1}{N-n}\left|D V\left(F_{s}\right) \cdot F_{s}^{\prime}\right|^{2}+\operatorname{Tr}\left[B_{s}^{2}\right]\right) d \mu_{0}
\end{aligned}
$$

by choosing $\epsilon=\frac{N-n}{n}>0$. The same theorem yields continuity of $e(s)$ on $[0,1]$, semiconvexity on $(0,1)$, and the distributional bound on $e^{\prime \prime}(s)$ given by

$$
\begin{aligned}
e^{\prime \prime}(s)-\frac{1}{N} e^{\prime}(s)^{2} & \geq \int_{M} \operatorname{Ric}^{(N, V)}\left(F_{s}^{\prime}, F_{s}^{\prime}\right) d \mu_{0} \\
& \geq K \int_{M} \ell^{2}\left(x, F_{1}(x)\right) d \mu_{0} \\
& \geq K \ell_{q}\left(\mu_{0}, \mu_{1}\right)^{2}
\end{aligned}
$$

where the second and third estimates follow from the lower bound $\operatorname{Ric}^{(N, V)} \geq$ $K g \geq 0$ in timelike directions and the $q$-separation $\left|F_{s}^{\prime}(x)\right|_{g}=\ell\left(x, F_{1}(x)\right)>0$ via Jensen's inequality. If $e(s)$ is infinite at either endpoint, we can apply the foregoing argument on any subinterval of $[0,1]$ having finite entropy at its endpoints to reach the desired conclusion.

Remark 6.7 (The restriction $K \geq 0$ ) Note that the preceding proof uses $K \geq 0$ only to pass from (66) to (67). 


\section{Relaxing separation from the null future}

Considerations henceforth have been restricted to $q$-geodesics whose endpoints $\left(\mu_{0}, \mu_{1}\right)$ are $q$-separated. In this chapter we relax this restriction, to allow endpoints which merely admit an $\ell^{q}$-optimal $\pi \in \Pi\left(\mu_{0}, \mu_{1}\right)$ with $\ell>0$ holding $\pi$-a.e. Corollary 7.5 asserts equivalence of timelike lower Ricci bounds to weak $(K, N, q)$ convexity of the relative entropy on the enlarged set of geodesics which arise in this more general setting.

Under these weaker hypotheses, we no longer know whether or not strong duality holds: i.e. we assume only that the dual infimum (33) is finite, but not that it is attained; see e.g. [20] and its references. Nevertheless, the following theorem decomposes the more general $\ell^{q}$-optimal measures $\pi$ which vanish on $\operatorname{sing}(\ell)$ into countably many components whose left and right marginals are $q$-separated (iii). This allows us to deduce (i) the uniqueness of $\pi$ and $\ell^{q}$-cyclical monotonicity of its support; (ii) the existence of Monge maps $F$; (iv) absolute continuity of $\mu_{s}$ along the corresponding $q$-geodesic. Our strategy for obtaining the existence and uniqueness results (i)-(ii) without dual attainment is inspired by Gigli's approach to a similar question in a less smooth setting [46]. The arguments of this section become somewhat simpler if one is satisfied to have results only for compactly supported measures

Theorem 7.1 (Maps characterizing interpolants without duality) Let $\left(M^{n}, g\right)$ be a globally hyperbolic spacetime. Fix $V \in C^{2}(M), 0<q<1$, $\mu \in \mathcal{P}^{a c}(M)$ and $\nu \in \mathcal{P}(M)$ for which the infimum (33) is finite. Then (i) at most one $\ell^{q}$-optimal $\pi \in \Pi(\mu, \nu)$ has the additional property that $\ell>0$ holds $\pi$-a.e. (ii) If such a joint measure exists, then $\pi=(i d \times F)_{\#} \mu$ for some map $F: \operatorname{spt} \mu \longrightarrow \operatorname{spt} \nu$ and $\pi[\operatorname{sing}(\ell)]=0$. (iii) Moreover, $\pi=\sum_{i=1}^{\infty} \pi^{i}$ decomposes into countably many non-negative, mutually singular measures such that $\cup_{i=1}^{\infty} \operatorname{spt} \pi^{i}$ is $\ell^{q}$-cylically monotone and the marginals $\left(\mu^{i}, \nu^{i}\right)$ of $\hat{\pi}^{i}:=\pi^{i} / \pi^{i}\left[M^{2}\right]$ have $\operatorname{spt}\left[\mu^{i} \times \nu^{i}\right]$ compact and disjoint from $\{\ell \leq 0\}$. For each $i \in \mathbf{N}$, the map $F$ agrees $\mu^{i}$-a.e. with the unique $\ell^{q}$-optimal map $F^{i}$ pushing $\mu^{i}$ forward to $\nu^{i}$ from Theorem 5.8; moreover $\operatorname{Graph}\left(F^{i}\right) \subset \operatorname{spt} \pi^{i}$. (iv) The q-geodesic $\left(\mu_{s}\right)_{s \in[0,1]}$ defined by $\mu_{s}:=\left(z_{s}\right)_{\#} \pi$ and (15) satisfies $\mu_{s} \in \mathcal{P}^{a c}(M)$ for $s<1$. (v) The measures $\mu_{s}^{i}:=\left(z_{s}\right)_{\#} \pi^{i}$ decompose $\mu_{s}$ into mutually singular pieces for $s<1$. (vi) The sum $\pi=\sum_{i} \pi^{i}$ is finite if and only if $\operatorname{spt} \pi$ is compact and disjoint from $\{\ell \leq 0\}$.

Proof. (iii)-(iv) and (vi): Suppose $\pi \in \Pi(\mu, \nu)$ is $\ell^{q}$-optimal and $\ell>0$ holds $\pi$-a.e. Since $M$ is a manifold and $\{\ell>0\}$ is open by Theorem 3.6, 
$\{\ell>0\} \cap \operatorname{spt} \pi$ can be covered by open rectangles $U \times W$ whose compact closures are contained in $\{\ell>0\}$. In fact, countably many such rectangles suffice due to the second countability of $M$; finitely many suffice if $\operatorname{spt} \pi$ is compact and contained in $\{\ell>0\}$. Setting $\pi^{0}=0$, define $\pi^{i}$ inductively as the restriction of $\pi-\pi^{i-1}$ to the $i$-th rectangle, so that $\pi=\sum_{i=1}^{\infty} \pi^{i}$, where the summands $\pi^{i}$ are mutually singular and each $\pi^{i}$ vanishes outside the $i$ th rectangle. Denote the marginals of $\pi^{i}$ by $\mu^{i}$ and $\nu^{i}$, and normalize $\hat{\pi}^{i}:=\pi^{i} / \pi^{i}\left[M^{2}\right]$ whenever $\pi^{i}$ is non-vanishing. Its marginals $\left(\hat{\mu}^{i}, \hat{\nu}^{i}\right)$ are $q$-separated by a pair of potentials $\left(u^{i}, v^{i}\right)$ according to Lemma 4.4, and $\pi^{i}$ and the partial sum $\sum_{k=1}^{i} \pi^{k}$ both inherit $\ell^{q}$-optimality from $\pi$ by e.g. Theorem 4.6 of [92, which requires finiteness of (33). Theorem 5.8 then asserts that $\pi^{i}=\left(i d \times F_{1}^{i}\right)_{\#} \mu^{i}$ and $\pi^{i}[\operatorname{sing}(\ell)]=0$, where $F_{s}^{i}=\exp s D H \circ D u^{i}$. Corollary 5.9 asserts that $\mu_{s}^{i}:=\left(z_{s}\right)_{\#} \pi^{i}$ is absolutely continuous for each $s<1$, establishing (iv). Compactness of $\operatorname{spt} \pi^{i}$ allows us to extend $F_{1}^{i}$ from $\operatorname{Dom} D u^{i}$ to spt $\mu^{i}$ so as to ensure $\operatorname{Graph}\left(F_{1}^{i}\right) \subset \operatorname{spt} \pi^{i}$. Since the support of $\sum_{k=1}^{i} \pi^{k}$ is compact, it lies a positive distance from the closed set $\{\ell \leq 0\}$, establishing (iv). Continuity of $\ell^{q}$ on a neighbourhood of $\cup_{k=1}^{i} \operatorname{spt} \pi^{k}$ ensures the latter is $\ell^{q}$-cyclically monotone by the well-known perturbation argument from my work with Gangbo [44]. Since $c$-cyclical monotonicity is checked on finite collections of points, it also holds for the limiting set $\cup_{k=1}^{\infty} \operatorname{spt} \pi^{k}$. Setting $\mu^{i j}:=\min \left\{\mu_{i}, \mu_{j}\right\}$, we next claim that $F_{1}^{i}=F_{1}^{j}$ holds $\mu^{i j}$-a.e.

To derive a contradiction suppose for some $i<j$ there is a set $S$ of positive measure for both $\mu^{i}$ and $\mu^{j}$ on which $F_{1}^{i} \neq F_{1}^{j}$. We may also suppose $\mu^{i}$ and $\mu^{j}$ to be given by densities with respect to $\operatorname{vol}_{g}$ which are bounded above and below on $S$. Take $S$ smaller if necessary to be compact, and so that for each $k \in\{i, j\}$, the map $F_{s}^{k}$ has approximate derivative $\tilde{D} F_{s}^{k}(x)$ depending smoothly on $s \in\left[0, \frac{1}{2}\right]$ and bounded above and below throughout $S$ in view of Proposition 6.1.

The compactness of $S$ ensures the existence of an $r$-neighbourhood $S^{r}$ of $S$ for some $r>0$ whose volume $\operatorname{vol}_{g}\left[S^{r}\right]<\frac{3}{2} \operatorname{vol}_{g}[S]$ is not much larger than that of $S$. Since the maps $F_{s}^{i / j}$ have bi-Lipschitz restrictions to $S$ for $s \leq 1 / 2$, stay far away from the cut locus, and coincide with the identity map when $s=0$, taking $s>0$ sufficiently small ensures that the compact sets $F_{s}^{i}(S)$ and $F_{s}^{j}(S)$ are contained in $S^{r}$ and both have volume larger than, say, $\frac{3}{4} \operatorname{vol}_{g}[S]$. Their intersection therefore has positive volume, so there exist $x, y \in S$ with $F_{s}^{i}(x)=F_{s}^{j}(x)$. By Proposition 5.1 this forces $x=y$ and $F_{1}^{i}(x)=F_{1}^{j}(y)$, since apart from a negligible set, the graphs of both $F_{1}^{i}$ and 
$F_{1}^{j}$ lie in the $\ell^{q}$-cyclically monotone set $\operatorname{spt}\left[\pi^{i}+\pi^{j}\right]$. This contradicts the definition of $S$, to establish (iii) that $F_{1}^{i}=F_{1}^{j}$ holds $\mu^{i j}$-a.e.

(i)-(ii) Now $F:=F^{i}$ is well-defined $\mu$-a.e. Since $\pi^{i}$ vanishes outside $\operatorname{Graph}(F) \cap \operatorname{sing}(\ell)$ for each $i$, we see $\pi=(i d \times F)_{\#} \mu$ by e.g. Lemma 3.1 of [2]. If there were a second $\ell^{q}$-optimal $\pi^{\prime} \in \Pi(\mu, \nu)$ with spt $\pi^{\prime}$ disjoint from $\operatorname{sing}(\ell)$, we could apply the foregoing argument to $\tilde{\pi}:=\left(\pi+\pi^{\prime}\right) / 2$ to deduce the existence of a map $\tilde{F}$ such that $\tilde{\pi}=(i d \times \tilde{F})_{\#} \mu$. Since both $\pi$ and $\pi^{\prime}$ vanish outside the graph of $\tilde{F}$, we conclude $\pi=(i d \times \tilde{F})_{\#} \mu=\pi^{\prime}$ as before, to establish the uniqueness of $\pi$.

(v) Fix $i \neq j$. Then $\mu^{i}$ and $\mu^{j}$ inherit mutual singularity from $\pi^{i}$ and $\pi^{j}$, because $(i d \times F)_{\#} \min \left\{\mu^{i}, \mu^{j}\right\}$ — being common to $\pi^{i}$ and $\pi^{j}$ — must vanish. Inner regularity provides disjoint $\sigma$-compact sets $U^{i} \subset$ spt $\mu^{i}$ such that

$$
\mu^{i}\left[U^{j}\right]=\left\{\begin{array}{cl}
\mu^{i}[M] & \text { if } i=j \\
0 & \text { else. }
\end{array}\right.
$$

We claim the $\left\{\mu_{s}^{i}\right\}_{j=1}^{\infty}$ remain mutually singular for each $s \in(0,1)$. Indeed, $\mu_{s}^{i}$ vanishes outside the $\sigma$-compact set $F_{s}\left(U^{i}\right)$, which we claim is disjoint from $F_{s}\left(U^{j}\right)$ unless $i=j$. Notice $z \in F_{s}\left(U^{i}\right) \cap F_{s}\left(U^{j}\right)$ implies $U^{i}$ intersects $U^{j}$ by Proposition 5.1 and the $c$-cyclical monotonicity of $\cup_{i=1}^{\infty} \operatorname{spt} \pi_{i}$. But this intersection forces $i=j$ to conclude the proof.

We next aim to establish expressions for the first two derivatives of the relative entropy $e(s):=E_{V}\left(\mu_{s}\right)$ along $q$-geodesics whose endpoints need not be $q$-separated, by extending Theorem 6.4 to the present setting. We extend the entropy $E_{V}(\mu)$ to subprobability measures by the same prescription (91) as for probability measures. The following pair of lemmas are known but included for completeness.

Lemma 7.2 (Domain of the relative entropy) Let $m$ and $\mu$ be Borel measures on a metric space $(M, d)$, with $\mu$ absolutely continuous with respect to $m$ and $\mu[M]<\infty$. Set

$$
E_{ \pm}(\mu \mid m):=\int_{M}\left[\frac{d \mu}{d m} \log \frac{d \mu}{d m}\right]_{ \pm} d m
$$

where $[a]_{ \pm}:=\max \{ \pm a, 0\}$. (i) If $0 \leq \nu \leq \mu$ and $E_{+}(\mu \mid m)$ (or $E_{-}(\mu \mid m)$ ) is finite, then $E_{+}(\nu \mid m)$ (respectively $\left.E_{-}(\nu \mid m)\right)$ is finite. If neither is finite then $E(\mu \mid m):=-\infty$; otherwise $E(\nu \mid m):=E_{+}(\nu \mid m)-E_{-}(\nu \mid m)$ satisfies

$$
-\mu[M]-E_{-}(\mu \mid m) \leq E(\nu \mid m) \leq E_{+}(\mu \mid m) .
$$


(ii) If $\mu=\sum_{i=1}^{\infty} \mu^{i}$ and the $\mu^{i}$ are mutually singular, then either $E(\mu \mid m)=$ $-\infty$ or $E(\mu \mid m)=\lim _{k \rightarrow \infty} E\left(\sum_{i=1}^{k} \mu^{i} \mid m\right)$.

Proof. (i) Fix Borel measures $0 \leq \nu \leq \mu$ and $m$ on $(M, d)$ with $\mu[M]<$ $\infty$ and $\mu$ absolutely continuous with respect to $m$. Let $\rho:=d \mu / d m$ and $\sigma:=d \nu / d m$ denote the Radon-Nikodym derivatives of $\mu$ and $\nu$ with respect to $m$. Since $\sigma \leq \rho$ and $\sigma \log \sigma \geq-1 / e$, if $r>0$ then

$$
\begin{aligned}
\int_{\{\rho>r\}} \sigma \log \sigma d m & \leq \int_{\{\rho>1\}} \rho \log \rho d m=E_{+}(\mu \mid m) \\
\text { and } \quad \int_{\{\rho>r\}} \sigma \log \sigma d m & \geq-\frac{\mu[M]}{e r}>-\infty
\end{aligned}
$$

by Chebyshev's inequality. This shows $E_{+}(\nu \mid m)$ is finite if $E_{+}(\mu \mid m)$ is. On the other hand, monotonicity of $\rho \log \rho$ on $[0,1 / e]$ yields

$$
0 \geq \int_{\left\{\rho \leq \frac{1}{e}\right\}} \sigma \log \sigma d m \geq \int_{\left\{\rho \leq \frac{1}{e}\right\}} \rho \log \rho d m \geq E_{-}(\mu \mid m) .
$$

Taking $r=1 / e$ we can sum these two estimates to conclude $E_{-}(\nu \mid m)$ is finite if $E_{-}(\mu \mid m)$ is, and obtain (70) unless both bounds diverge.

(ii) Let $\sigma:=d \mu / d m$ and $\sigma^{i}:=d \mu^{i} / d m$. Since the $\mu^{i} \geq 0$ are mutually singular and $\mu=\sum \mu_{i}$ is absolutely continuous with respect to $m$, for $m$-a.e. $x$ only one of the three inequalities $0 \leq \sigma^{k}(x) \leq \sigma^{k+1}(x) \leq \sigma(x)$ can be strict. Thus

$$
\begin{aligned}
\lim _{k \rightarrow \infty} \int_{\{\sigma>1\}} \sigma^{k} \log \sigma^{k} d m & =\int_{\{\sigma>1\}} \sigma \log \sigma d m \\
\text { and } \lim _{k \rightarrow \infty} \int_{\{\sigma \leq 1\}} \sigma^{k} \log \sigma^{k} d m & =\int_{\{\sigma \leq 1\}} \sigma \log \sigma d m
\end{aligned}
$$

follow from Lebesgue's monotone convergence theorem, establishing (ii).

Lemma 7.3 (Consequences of Helly's selection theorem) Given $c \in$ $\mathbf{R}$ and a sequence of convex functions $f_{k}:[0,1] \longrightarrow[-\infty, c]$, a subsequence $f_{k(j)}$ converges pointwise a.e. to a convex limit $f:[0,1] \longrightarrow[-\infty, c]$ satisfying either

$$
\begin{aligned}
& \inf _{0 \leq s \leq 1} f(s)>-\infty \quad \text { (proper) } \\
& \text { or } \sup _{0<s<1} f(s)=-\infty \quad \text { (improper). }
\end{aligned}
$$


In the proper case, the derivatives $f^{\prime}=\lim _{j \rightarrow \infty} f_{k(j)}^{\prime}$ converge pointwise a.e. and the second derivatives $f^{\prime \prime}=\lim _{j \rightarrow \infty} f_{k(j)}^{\prime \prime}$ converge distributionally on $(0,1)$.

Proof. The proof is standard, hence omitted.

Theorem 7.4 (Displacement Hessian of the relative entropy again) Let $\left(M^{n}, g\right)$ be a globally hyperbolic spacetime. Fix $V \in C^{2}(M), N>n$, and $0<q<1$. Fix $\mu, \nu \in \mathcal{P}^{a c}(M)$ for which the infimum (33) is finite and the supremum (6) is attained by some $\pi \in \Pi(\mu, \nu)$ with $\ell>0$ holding $\pi$ a.e. Assume the relative entropy $e(s):=E_{V}\left(\mu_{s}\right)$ wth $\mu_{s}:=z_{s \#} \pi$ and map $F_{s}(x):=z_{s}(x, F(x))$ from Theorem 7.1 satisfy $\max \{e(0), e(1)\}<\infty$ and $\sup _{0<s<1} e(s)>-\infty$ and

$$
C:=\left\|\int_{M} \min \left\{\operatorname{Ric}_{F_{s}(x)}^{(N, V)}\left(\frac{\partial F}{\partial s}, \frac{\partial F}{\partial s}\right), 0\right\} d \mu_{s}\right\|_{L^{\infty}([0,1])}<\infty .
$$

Then the conclusions of Theorem 6.4(a) remain true, except that $e(\cdot)$ may be upper semicontinuous rather than continuous at the the endpoints of the interval $s \in[0,1]$.

Proof. Fix $\mu, \nu \in \mathcal{P}^{a c}(M)$ and $\pi \in \Pi(\mu, \nu)$ as described. Let the map $F$, q-geodesic $\left(\mu_{s}\right)_{s \in[0,1]} \subset \mathcal{P}^{a c}(M)$ and mutually singular decompositions $\pi=\sum_{i=1}^{\infty} \pi^{i}$ and $\mu_{s}:=\sum \mu_{s}^{i}$ with $\mu_{s}^{i}:=z_{s \#} \pi^{i}$ and $\ell^{q}$-cyclically monotone spt $\pi^{i} \subset\{\ell>0\}$ be given by Theorem [7.1, which also asserts $\pi[\operatorname{sing}(\ell)]=0$. Normalizing $\hat{\mu}^{i}:=\mu^{i} / \mu^{i}[M]$ and defining $\hat{\nu}^{i}$ and $\hat{\pi}^{i}$ analogously, the marginals $\left(\hat{\mu}^{i}, \hat{\nu}^{i}\right)$ of $\hat{\pi}^{i}$ are $q$-separated by Lemma 4.4, and $F$ coincides a.e. with the unique optimal map between them provided by Theorem 5.8, so $\hat{\pi}^{i}$ is $\ell^{q}$ optimal. Moreover, $\mu^{i}:=\mu_{0}^{i}$ and $\nu^{i}:=\mu_{1}^{i}$ inherit an upper bound on their entropy from $\max \{e(0), e(1)\}<\infty$ by Lemma 7.2 , being compactly supported they inherit a lower bound on their entropy from (10). Their normalized versions also have finite entropy according to the scaling law

$$
E_{V}(\lambda \nu)=\lambda E_{V}(\nu)+\nu[M] \lambda \log \lambda
$$

for $\lambda>0$. Setting $\hat{e}_{i}(s):=E_{V}\left(\hat{\mu}_{s}^{i}\right)$ and applying Theorem 6.4 to $\left(\hat{\mu}_{0}^{i}, \hat{\mu}_{1}^{i}\right)$ yields

$$
\begin{aligned}
e_{i}^{\prime}(s) & =\int_{M}\left[D V_{F_{s}(x)} F_{s}^{\prime}(x)-\operatorname{Tr} B_{s}(x)\right] d \mu_{0}^{i}(x) \quad \text { and } \\
e_{i}^{\prime \prime}(s) & =\int_{M}\left[\operatorname{Tr}\left(B_{s}^{2}(x)\right)+\left(\operatorname{Ric}+D^{2} V\right)_{F_{s}(x)}\left(F_{s}^{\prime}(x), F_{s}^{\prime}(x)\right)\right] d \mu_{0}^{i}(x)
\end{aligned}
$$


on $s \in(0,1)$ with and therefore also without the hats denoting normalization.

The mutual singularity of $\left\{\mu_{s}^{i}\right\}_{i=1}^{\infty}$ asserted by Theorem 7.1 extends to $s=1$ by the $s \leftrightarrow 1-s$ symmetry. We can therefore obtain (54)-(55) by summing (77)-(78) over $i \in \mathbf{N}$ - provided these sums do not diverge. More precisely, define $f_{k}(s):=\sum_{i=1}^{k} e_{i}(s)$. Hypothesis (75) combines with $\operatorname{Tr}\left(B_{s}(x)^{2}\right) \geq 0$ from Theorem [6.4 in (178) to show $\frac{C}{2} s^{2}+f_{k}(s)$ is convex on $[0,1]$. Lemma 7.2 shows $f_{k}(0)$ and $f_{k}(1)$ to be bounded above in terms of $C$ and the endpoints $\mu$ and $\nu$, and asserts for each $s \in[0,1]$ that either $e(s):=E_{V}\left(\mu_{s}\right)=\lim _{k \rightarrow \infty} f_{k}(s)$ or $e(s)=-\infty$. We assert:

Claim 1: If $e(s)=-\infty$ for some $s \in(0,1)$ then $\sup _{0<t<1} e(t)=-\infty$.

Taking Claim 1 for granted (and postponing its proof), if $e(t) \neq-\infty$ for some $t \in(0,1)$, then Claim 1 yields $e(s) \neq-\infty$ for all $s \in(0,1)$, hence pointwise convergence of the full sequence $\frac{C}{2}|\cdot|^{2}+f_{k}(\cdot)$ to the limit $\frac{C}{2}|\cdot|^{2}+e(\cdot)$, which must be convex and real-valued by Lemma 7.3. The same lemma asserts $e^{\prime}(s)=\lim _{k \rightarrow \infty} f_{k}^{\prime}(s)$ pointwise a.e. and $e^{\prime \prime}(s)=\lim _{k \rightarrow \infty} f_{k}^{\prime \prime}(s)$ distributionally on $(0,1)$.

Unless $(0,1) \subset e^{-1}(-\infty)$, (55) therefore follows by summing (78) using Lebesgue's monotone convergence theorem and the pointwise lower bound established above showing its integrand $\geq-C$. Integrating (55) yields (54) and its continuous dependence on $s \in(0,1)$ exactly as in the proof of Theorem 6.4. To complete the proof of the theorem, it remains only to verify Claim 1, which we do in a series of subclaims.

Proof of Claim 1: Let $\rho_{t}:=d \mu_{t} / d m$ denote the Radon-Nikodym derivative of $d \mu_{t}(x)$ with respect to the weighted Lorentzian volume $d m(x)=$ $e^{-V(x)} d \operatorname{vol}_{g}(x)$. Set $N^{i}:=\left(\operatorname{spt} \pi^{i}\right) \backslash \operatorname{sing}(\ell)$ and $N^{\infty}=\cup_{i=1}^{\infty} N^{k}$. Assume $e(s)=-\infty$ for some fixed value of $s \in(0,1)$, since otherwise there is nothing to prove. To establish $e(t)=-\infty$ for all other $t \in(0,1)$, observe $z_{t}: N^{\infty} \longrightarrow M$ is smooth by Lemma 2.4 and its inverse map is countably Lipschitz on $z_{t}\left(N^{\infty}\right)$ by Theorem 5.3. Since $N^{\infty}$ carries the full mass of $\pi$, inner regularity of $\mu_{s}$ yields a $\sigma$-compact $U$ of $\left\{z \in z_{s}\left(N^{\infty}\right) \mid \rho_{s}(z) \leq 1\right\}$ which differs from the latter by a $\mu_{s}$ negligible set. Let $\bar{\pi}^{i}$ denote the restriction of $\pi^{i}$ to the $\sigma$-compact set $S:=z_{s}^{-1}(U)$ with the convention $\pi^{\infty}:=\pi$. Set $\bar{\mu}_{t}^{i}:=z_{t \#}\left(\bar{\pi}^{i}\right)$ and $\bar{\nu}_{t}^{i}:=\sum_{j=1}^{i} \bar{\mu}_{t}^{j}$ for each $i \in \mathbf{N} \cup\{\infty\}$ and $t \in[0,1]$, and denote their entropies by $\bar{e}_{i}(t):=E_{V}\left(\bar{\mu}_{t}^{i}\right)$ and $\bar{f}_{i}(t):=E_{V}\left(\bar{\nu}_{t}^{i}\right)$. Then $0 \leq \bar{\mu}_{t}^{i} \leq \mu_{t}^{i}$ inherit absolute continuity and mutual singularity from $\left\{\mu_{t}^{i}\right\}_{i \in \mathbf{N}}$ so $\bar{f}_{k}(t)=\sum_{i=1}^{k} \bar{e}_{i}(t)$. 
Claim 2: Setting $\rho_{t}^{\infty}=\rho_{t}$, the following identity holds $m$-a.e.:

$$
\bar{\rho}_{t}^{i}:=\frac{d \bar{\mu}_{t}^{i}}{d m}=1_{z_{t}(S)} \rho_{t}^{i}, \quad \forall t \in(0,1) \text { and } i \in \mathbf{N} \cup\{\infty\}
$$

Proof of Claim 2: Absolute continuity of $\mu_{s}$ implies that $U=z_{s}(S)$ has either full or zero Lebesgue density $\mu_{s}$-a.e. Since $z_{t}$ is countably biLipschitz on $S$, and $\mu_{s}=z_{s \#} \pi$, it follows that $S$ has either full or zero $n$-dimensional density in $N^{\infty}$ (or alternately, in the $n$-dimensional Lipschitz submanifold $W \subset M \times M$ guaranteed to contain $N^{\infty}$ (hence spt $\pi$ ) by my result proved with Pass and Warren 68, which relies on the non-degeneracy of $\ell^{q}$ asserted in Corollary 3.7). In fact, absolute continuity of $\pi$ in coordinates on $W$ also follows from that of $\rho_{s}$ and the countably biLipschitz character of $z_{s}$; thus we may say $S$ has full or zero density Lebesgue density $\pi$-a.e. on $W$. Similarly, it follows that $z_{t}(S)$ has either full or zero Lebesgue density $\mu_{t}:=z_{t \#} \pi$ a.e. for each $t \in(0,1)$ to establish claim 2 .

Claim 3: If $e(s)=-\infty$ then $\lim _{k \rightarrow \infty} \bar{f}_{k}(s)=-\infty$.

Proof of Claim 3: The absolute continuity and mutual singularity of $\bar{\mu}_{t}^{i} \leq \mu_{t}^{i}$ and our choice of $U=z_{s}(S)$ imply

$$
\begin{aligned}
-\infty=e(s) & =\int_{M} \rho_{s} \log \rho_{s} d m \\
& \geq \int_{\left\{\rho_{s} \leq 1\right\}} \rho_{s} \log \rho_{s} d m \\
& =\sum_{i=1}^{\infty} \int_{z_{s}(S)} \rho_{s}^{i} \log \rho_{s}^{i} d m \\
& =\lim _{k \rightarrow \infty} \bar{f}_{k}(s)
\end{aligned}
$$

to establish Claim 3.

Claim 4: If $e(s)=-\infty$ for some $s \in(0,1)$ then $\sup \lim \sup \bar{f}_{k}(t)=-\infty$. $0<t<1 \quad k \rightarrow \infty$

Proof of Claim 4: Let $\hat{\bar{\pi}}^{i}:=\bar{\pi}^{i} / \bar{\pi}^{i}[M]$ and normalize $\hat{\bar{\mu}}_{t}^{i}$ similarly. Now $\bar{\pi}^{i} \leq \pi^{i}$ implies $\hat{\bar{\pi}}^{i}$ inherits $\ell^{q}$-optimality from $\hat{\pi}^{i}$, and its marginals $\left(\hat{\bar{\mu}}_{0}^{i}, \hat{\bar{\mu}}_{1}^{i}\right)$ inherit $q$-separation from those of $\hat{\pi}^{i}$. Thus $\left\{\hat{\bar{\pi}}_{t}^{i}\right\}_{t \in[0,1]}$ is a $q$-geodesic for each $i$ and Theorem 6.4 shows convexity of $\frac{C}{2} t^{2}+\bar{f}_{i}(t)$ on $t \in[0,1]$ using (76) . Lemma 7.2 bounds $\bar{f}_{i}(0)$ and $\bar{f}_{i}(1)$ above in terms of $\mu, \nu$ and $C$. Defining $\bar{f}(t):=\limsup _{i \rightarrow \infty} f_{i}(t)$, claim 3 yields $\bar{f}(s)=-\infty$ hence Lemma 7.3 implies $\sup _{0<t<1} \bar{f}(t)=-\infty$ to establish Claim 4 . 
Claim 5: If $e(s)=-\infty$ for some $s \in(0,1)$ then $\sup _{0<t<1} e(t)=-\infty$.

Proof of claim 5: Claim 2 yields

$$
\begin{aligned}
e(t) & =\int_{M} \rho_{t} \log \rho_{t} d m \\
& =\int_{z_{t}(S)} \bar{\rho}_{t} \log \bar{\rho}_{t} d m+\int_{M \backslash z_{t}(S)} \rho_{t} \log \rho_{t} d m .
\end{aligned}
$$

The first summand coincides with $\bar{f}_{\infty}(t):=E_{V}\left(\bar{\mu}_{t}^{\infty}\right)$, which diverges to $-\infty$ by Lemma 7.2 combined with Claim 4 . Thus $e(t)=-\infty$ by the convention from Definition 1.2, regardless of whether or not the other integrals are welldefined. This establishes Claims 1 and 5, hence the theorem.

The following result provides an analog to Corollary 6.6. We obtain weak rather than strong $(K, N, q)$ convexity in this setting since we do not know whether or not there are other $\ell^{q}$-optimal measures $\pi \in \Pi(\mu, \nu)$ for which $\ell>0$ fails to hold $\pi$-a.e. If such measures exist, they generate $q$-geodesics via Theorem 2.11 which we have not developed the machinery to analyze.

Corollary 7.5 (Weak convexity from timelike lower Ricci bounds) Let $\left(M^{n}, g\right)$ be a globally hyperbolic spacetime. Fix $V \in C^{2}(M)$ bounded, $N>n$ and $0<q<1$. If $\operatorname{Ric}^{(N, V)}(v, v) \geq K|v|_{g}^{2} \geq 0$ holds in every timelike direction $(v, x) \in T M$, then the relative entropy $E_{V}(\mu)$ of (9) is weakly $(K, N, q)$-convex for the set $Q \subset \mathcal{P}^{a c}(M)^{2}$ of measures $(\mu, \nu)$ having infimum (33) finite and supremum (6) attained by some $\pi \in \Pi(\mu, \nu)$ with $\ell>0$ holding $\pi$-a.e.

Proof. The proof of this corollary follows from Theorem 7.4 exactly as Corollary 6.6 follows from Theorem 6.4(a); we may take $C=0$ due to our hypothesized timelike lower Ricci curvature bound.

The only difference is that, for non-compactly supported measures, we do not have the a priori lower bound (10) on $e(s):=E_{V}\left(\mu_{s}\right)$, where $\left(\mu_{s}\right)_{s \in[0,1]} \subset$ $\mathcal{P}^{a c}(M)$ is the $q$-geodesic with endpoints $(\mu, \nu) \in Q$ provided by Theorem 7.4 . However, as long as $\max \{e(0), e(1)\}<\infty$, the convexity of $e(s)$ established in that theorem ensures $e(s)$ is real-valued unless $\sup _{0<t<1} e(t)=-\infty$. If $\max \{e(0), e(1)\}=+\infty$, we can apply the foregoing argument on any subinterval $\left[t_{0}, t_{1}\right] \subset[0,1]$ satisfying $\max \left\{e\left(t_{0}\right), e\left(t_{1}\right)\right\}<\infty$ to conclude that $e(s)$ is real-valued, convex and satisfies the desired estimates on $\left[t_{0}, t_{1}\right]$ unless $\left(t_{0}, t_{1}\right) \subset e^{-1}(-\infty)$. Either way, we obtain the weak $(K, N, q)$ convexity from Definition 6.5 of $E_{V}$ for $Q$. 


\section{Ricci lower bounds from entropic convexity}

This final section is devoted to establishing converses to the corollaries of the preceding sections, by constructing a $q$-geodesic which shows the sufficient conditions for $(K, N, q)$-convexity of $E_{V}$ they provide are also necessary. The strategy is based on developing a Lorentzian analog for constructions given in the Riemannian setting by von Renesse and Sturm 80, and generalized by Sturm [85], Lott and Villani 62]. It culminates in Theorem 8.5, which

produces a $q$-geodesic along which this convexity fails in the absence of the appropriate timelike lower Ricci curvature bound.

Lemma 8.1 (Hessian of the Lorentz distance) Let $\left(M^{n}, g\right)$ be a globally hyperbolic Lorentzian manifold. Fix $0<q<1$ and a future-directed proper-time parameterized geodesic segment $t \in\left[0, t_{0}\right] \mapsto y(t) \in M$. Then

$$
\begin{aligned}
-\frac{\partial^{2}}{\partial x^{\alpha} \partial x^{\beta}} \ell(x, y(t) ; q) & =\frac{\partial^{2} L}{\partial v^{\alpha} \partial v^{\beta}}\left(t y^{\prime}(t), y(t) ; q\right)+O\left(t^{q}\right) \\
& =O\left(t^{q-2}\right)
\end{aligned}
$$

as $t \rightarrow 0^{+}$, where the derivatives are taken in Fermi coordinates along the geodesic segment in question and the Hessian of $L$ is positive definite.

Proof. Recall that Fermi coordinates both flatten the geodesic $y(t)$ and act as Lorentzian normal coordinates at each point along it. Given $0 \neq w \in$ $T_{x} M$, set $x(s)=\exp _{y(0)} s w$ and let $\gamma_{(s, t)}:[0,1] \longrightarrow M$ denote the propertime maximizing geodesic joining $\gamma_{(s, t)}(0)=x(s)$ to $\gamma_{(s, t)}(1)=y(t)$. Taking two derivatives of

$$
-\frac{1}{q} \ell(x(s), y(t))^{q}=\int_{0}^{1} L\left(\dot{\gamma}_{(s, t)}(\lambda) ; q\right) d \lambda=\frac{1}{q} \int_{0}^{1}\left|\dot{\gamma}_{s, t}(\lambda)\right|^{q} d \lambda
$$

and using $\left.\frac{\partial^{2} x^{\alpha}}{\partial s^{2}}\right|_{s=0}=0$ leads to

$$
\begin{aligned}
-w^{\alpha} w^{\beta} \frac{\partial^{2}}{\partial x^{\alpha} \partial x^{\beta}} \ell(x, y(t) ; q) & =\int_{0}^{1}\left[D^{2} L \frac{\partial \dot{\gamma}}{\partial s} \frac{\partial \dot{\gamma}}{\partial s}+D L \frac{\partial^{2} \dot{\gamma}}{\partial s^{2}}\right]_{s=0} d \lambda \\
& =\int_{0}^{1} D^{2} L(x, y(\lambda) ; q) \frac{\partial \dot{\gamma}}{\partial s} \frac{\partial \dot{\gamma}}{\partial s} d \lambda,
\end{aligned}
$$

where the $D L$ integral vanishes (after integrating by parts) by the geodesy of $\gamma=\gamma_{(s, t)}$, and the facts that one endpoint $\gamma_{(s, t)}(1)=y(t)$ is independent 
of $s$ while the other $\gamma_{(s, t)}(0)=x(s)$ is a geodesic whose second $s$ derivative vanishes in our chosen coordinates.

Since the Lorentzian geodesic $\gamma_{(s, t)}$ depends smoothly on its endpoints, $\frac{\partial \gamma}{\partial s}$ is a Jacobi fields along $\gamma_{(0, t)}$ with end vectors $w$ and 0 . Since the geodesics in question are collapsing to a point where the geometry is asymptotic to Minkowski space, these Jacobi fields are asympotically linear. The intermediate value theorem and Jacobi equation yield

$$
\frac{\partial \dot{\gamma}_{(0, t)}^{\alpha}}{\partial s}(\lambda)=w^{\alpha}+O\left(w t^{2}\right) .
$$

Inserting $v=\dot{\gamma}_{(0, t)}(\lambda)=t y^{\prime}(t)$ hence $|v|=t$ into (22) yields

$$
\int_{0}^{1} D^{2} L \frac{\partial \dot{\gamma}}{\partial s} \frac{\partial \dot{\gamma}}{\partial s} d \lambda=t^{q-2}\left[(2-q)\left(\left\langle y^{\prime}(t), w\right\rangle_{g}^{2}+|w|_{g}^{2}\right]\left(1+O\left(t^{2}\right)\right)\right.
$$

where the quantity in square brackets is positive due to the the uniform convexity of $L$ proved in Lemma 3.1. Comparison with (81) yields the claims of the present lemma.

Corollary 8.2 (Local concavity of the Lorentz distance) The hypotheses and terminology of Lemma 8.1 imply the second Lorentzian derivative of $\ell(x, y(t))^{q}$ with respect to $x$ is negative-definite for $t>0$ sufficiently small.

Proof. Apart from its sign, the left-hand side of (79) gives the second covariant derivative in question. For $t>0$ sufficiently small, the equated right-hand side becomes positive-definite by uniform convexity of $L$ proved in Lemma 3.1.

By Lemma 8.1 and Corollary 3.7, choosing $(\bar{p}, \bar{x}) \in T^{*} M$ non-zero, timelike, past-directed and sufficiently small ensures $\ell^{q}(\cdot, \bar{y})$ is non-degenerate with Hessian $D^{2} \ell^{q}(\bar{x}, \bar{y})<0$ at $\bar{x}$, where $\bar{y}=\exp _{\bar{x}} D H(\bar{p}, \bar{x} ; q)$. The next proposition provides an $\frac{\ell^{q}}{q}$-convex function $u=u_{\tilde{q} q}$ which is smooth on a neighbourhood $U$ of $\bar{x}$ and satisfies $D u(\bar{x})=\bar{p}$ and $D^{2} u(\bar{x})=0$. As the remark following indicates, the proof works in greater generality than stated.

Lemma 8.3 (Prescribing the 2-jet of an $\frac{\ell^{q}}{q}$-convex function at $\bar{x}$ ) Fix $0<q<1$, a compact set $X \times Y \subset M \times M \backslash \operatorname{sing}(\ell)$ with $(\bar{x}, \bar{y})$ in its interior, and a smooth function $u$ satisfying the first- and second-order conditions $D u(\bar{x})=D_{x} b(\bar{x}, \bar{y})$ and $D^{2} u(\bar{x})>D_{x x}^{2} b(\bar{x}, \bar{y})$ strictly, where $b:=\frac{1}{q} \ell^{q}$. Then there is a b-convex function $w$ on $X$ which agrees with $u$ in some neighbourhood of $\bar{x}$. 
Proof: Two applications of the implicit function theorem show that the relation $D_{x} b(x, y)-D u(x)=0$ defines a diffeomorphic correspondence $F$ between $x$ and $y$ near $(\bar{x}, \bar{y})$ : the non-degeneracy of $b$ from Corollary 3.7(iii) gives $y=F(x)$ locally as a graph over $x$; conversely, $F$ is smoothly invertible since $D^{2} u(\bar{x})-D_{x x}^{2} b(\bar{x}, \bar{y})$ has full rank. Use this correspondence to define $v$ near $\bar{y}=F(\bar{x})$ so that $v(F(x))=b(x, F(x))-u(x)$. On a small enough neighbourhood $U \times F(U)$ of $(\bar{x}, \bar{y})$, the second-order hypothesis implies for each $y \in F(U)$ that $x \in U \mapsto u(x)+v(y)-b(x, y)$ has no critical points save the local minimum $x=F^{-1}(x)$ at which it vanishes. In other words $u(x)+v(y)-b(x, y)$ is non-negative on $U \times F(U)$ and vanishes on the graph of the diffeomorphism $F: U \longrightarrow F(U)$. Then

$$
w(x):=\sup _{y \in F(U)} b(x, y)-v(y)
$$

defines the desired $b$-convex function and coincides with $u$ throughout $U$.

Remark 8.4 Adopting the usual definion of b-convexity from e.g. [82], the preceding proposition and proof extend immediately to any smooth cost function $-b(x, y)$ on a compact product $X \times Y$ of equal dimensional manifoldswith-boundary satisfying the non-degeneracy condition det $D_{x^{i} y^{j}}^{2} b(\bar{x}, \bar{y}) \neq 0$. No other properties specific to the Lorentz distance have been used.

Theorem 8.5 (Entropic convexity implies a timelike Ricci bound) Let $\left(M^{n}, g\right)$ be a globally hyperbolic spacetime. Fix $V \in C^{2}(M), K \in \mathbf{R}$ and $N \neq n$. If $\operatorname{Ric}^{(N, V)}(v, v) \geq K|v|_{g}^{2}$ fails at some timelike vector $(v, x) \in T M$, then the relative entropy $E_{V}(\mu)$ of (9) fails to be weakly $(K, N, q)$-convex for any $0<q<1$. In fact, the q-geodesic $s \in[0,1] \mapsto \mu_{s} \in \mathcal{P}_{c}^{a c}(M)$ along which $\left(\ell_{q}\left(\mu_{0}, \mu_{1}\right)^{2} K, N\right)$ convexity of $e(s):=E_{V}\left(\mu_{s}\right)$ fails may be constructed so that $e \in C^{2}([0,1])$, and $\operatorname{spt}\left[\mu_{0} \times \mu_{1}\right]$ is disjoint from $\{\ell \leq 0\}$ but contained in an arbitrarily small neighbourhood of $(x, x)$.

Proof. Suppose $\operatorname{Ric}^{(N, V)}(\hat{v}, \hat{v})<K \in \mathbf{R}$ at some future-directed vector $(\hat{v}, \bar{x}) \in T M$ with $|\hat{v}|_{g}=1$. The idea of the proof is to construct a $q$-geodesic starting from measure $\mu_{0}$ which is concentrated (say uniformly) within a (Riemannian) ball of radius $r$ around $\bar{x}$, and to transport it in the direction $\hat{v}$ for proper-time $t$, where $r \ll t \ll 1$ are chosen sufficiently small that the Ricci curvature remains approximately constant along the geodesic, to facilitate computation and to contradict the $(K, N, q)$ convexity of $E_{V}(\mu)$. The 
transport will be generated by a smooth potential $u=u_{\tilde{q} q}$ whose first two derivatives at $\bar{x}$ may be freely prescribed within limits imposed by Proposition 8.3. Once $D u(\bar{x})$ has been selected to transport $\bar{x}$ to $y_{t}:=\exp _{\bar{x}} t \hat{v}$, we'll choose $D^{2} u(\bar{x})$ to make the product $D^{2} H D^{2} u$ from (44) become a suitable multiple of the identity operator on $T_{\bar{x}} M$, thus achieving the case of equality in certain inequalities in the proof.

The construction, which is localized at $\bar{x}$, will be carried out in Fermi coordinates around the geodesic $y_{t}=\exp _{\bar{x}} t \hat{v}$. Lemma 8.1 provides $t>0$ sufficiently small that the Hessian of $x \mapsto \ell^{q}\left(x, y_{t}\right)$ at $\bar{x}$ is negative-definite and satisfies

$$
\begin{aligned}
O\left(t^{q-1}\right) & = \pm t \frac{(D V(\bar{x}) \hat{v})}{N-n} \frac{\partial^{2} L}{\partial v^{\alpha} \partial v^{\beta}}(\bar{x}, t \hat{v} ; q) \\
& >\frac{\partial^{2} \ell}{\partial x^{\alpha} \partial x^{\beta}}\left(\bar{x}, y_{t} ; q\right) \\
& =O\left(t^{q-2}\right)
\end{aligned}
$$

plus the non-degeneracy condition of Corollary 3.7. Fix $v_{t}:=t \hat{v}$ and $p_{t}:=$ $D L\left(v_{t}, \bar{x} ; q\right)$; since we are inside the cut locus we know $H$ is smooth at $\left(p_{t}, \bar{x}\right)$ and $v_{t}=D H\left(p_{t}, \bar{x} ; q\right)$. Since $y_{t}$ lies in the future of $\bar{x}$ but within the timelike cut locus, there is a compact neighbourhood $X \times Y$ of $\left(\bar{x}, y_{t}\right)$ which is disjoint from $\operatorname{sing}(\ell)$. Proposition 8.3 provides an $\frac{\ell^{q}}{q}$-convex $u=u_{\tilde{q} q} \in$ $C^{3}$ with $D u(\bar{x})=p_{t}$ and $D^{2} u(\bar{x})=-\frac{1}{N-n}\left(D V(\bar{x}) v_{t}\right) D^{2} H\left(p_{t}, \bar{x} ; q\right)^{-1}$ where $D^{2} H\left(p_{t}, \bar{x} ; q\right)^{-1}=D^{2} L\left(v_{t}, \bar{x} ; q\right)$ from Lemma 3.1 has been exploited. Thus $F_{s}(x):=\exp _{x} s D H(D u(x), x ; q)$ is $C^{2}$ and $y_{t}=F_{1}(\bar{x})$.

Take $\mu_{0}^{(r)}$ to be the uniform distribution (with respect to $\operatorname{vol}_{g}$ say) over the Riemannian ball $X_{r}:=\tilde{B}_{r}(\bar{x})$, so that $\mu_{0}^{(r)} \rightarrow \delta_{\bar{x}}$ against continuous test functions. For $r>0$ sufficiently small, $X_{r} \times F_{1}\left(X_{r}\right) \subset X \times Y$ hence disjoint from sing $(\ell)$. Lemma 4.4 combines with Theorem 5.8 and its corollary to show $\mu_{s}^{(r)}:=F_{s \#} \mu_{0}^{(r)} \in \mathcal{P}_{c}^{a c}(M)$ defines the unique $q$-geodesic on $s \in[0,1)$ connecting its endpoints. Moreover

$$
\lim _{r \rightarrow 0} \ell_{q}\left(\mu_{0}^{(r)}, \mu_{1}^{(r)}\right)=\left|v_{t}\right|_{g}=t .
$$

Regarding $r>0$ as fixed for the moment, let $\rho_{s}:=d \mu_{s}^{(r)} / d \operatorname{vol}_{g}$ and $e(s ; r):=$ $E_{V}\left(\mu_{s}^{(r)}\right)$ denote the relative entropy along the geodesic in question. Since $u$ is smooth, for $s<1$ the Monge-Ampère type equation of Corollary 5.11 
bounds $\left\|\rho_{s}\right\|_{\infty}$ in terms of $\left\|\rho_{0}\right\|_{\infty}$. Thus $e(0 ; r)$ and $e(s ; r)$ are finite, and Theorem 6.4 yields $e(\cdot ; r)$ continuous and semiconvex on $s \in[0,1)$. Moreover, smoothness of $F_{s}(x)$ implies the terms $B_{s}(x)=D F_{s}^{\prime}(x) D F_{s}(x)^{-1}$ which appear in (54)-(55) depend continuously on $(s, x) \in[0,1) \times M$. Thus Lebesgue's dominated convergence theorem yields $e(\cdot ; r) \in C^{2}([0,1))$ with Theorem 6.4 and Lemma 5.7 giving its first two $s$ derivatives

$$
\begin{aligned}
e^{\prime}(0 ; r) & =\int_{M}\left[D V D H(D u)-H^{i j} u_{j i}\right] d \mu_{0}^{(r)} \\
& \rightarrow\left(1+\frac{n}{N-n}\right) D V(\bar{x}) v_{t} \quad \text { as } r \rightarrow 0 \\
e^{\prime \prime}(0 ; r) & =\int_{M}\left[H^{i j} u_{j k} H^{k l} u_{l i}+\left(\operatorname{Ric}+D^{2} V\right)(D H(D u), D H(D u))\right] d \mu_{0}^{(r)} \\
& \rightarrow \frac{n}{(N-n)^{2}}\left(D V(\bar{x}) v_{t}\right)^{2}+\operatorname{Ric}^{(N, V)}\left(v_{t}, v_{t}\right)+\frac{1}{N-n}\left(D V(\bar{x}) v_{t}\right)^{2},
\end{aligned}
$$

in view of (11). Thus

$$
\begin{aligned}
\lim _{r \rightarrow 0} e^{\prime \prime}(0 ; r)-\frac{1}{N} e^{\prime}(0 ; r)^{2} & =\operatorname{Ric}^{(N, V)}\left(v_{t}, v_{t}\right)^{2} \\
& <K\left|v_{t}\right|_{g}^{2} \\
& =K \lim _{r \rightarrow 0} \ell_{q}\left(\mu_{0}^{(r)}, \mu_{1}^{(r)}\right)^{2}
\end{aligned}
$$

For $r>0$ sufficiently small, this contradicts $\left(K \ell_{q}\left(\mu_{0}^{(r)}, \mu_{1}^{(r)}\right)^{2}, N\right)$ convexity of $e(s ; r)$ on $[0,1)$, as desired.

\section{A Monge-Mather shortening estimate}

This appendix contains the deferred proof of Theorem 5.3, which we restate for convenience below. If the Lagrangian (20) were smooth and uniformly convex, this would follow from Corollary 8.2 of the Monge [71]-Mather [63] shortening principal from [92]; see also [25]. However, things are made delicate by the fact that both smoothness and uniform convexity of our Lagrangian $L(v, x ; q)$ degenerate at the light cone (Lemma 3.1). Inspired by [36] and Theorem 8.23 of [92], we use compactness and the $q$-separation hypothesis to derive the desired Lipschitz continuity directly. For $q=1$, related estimates are established by Suhr [88]. 
Theorem A.1 (Lipschitz inverse maps) Fix $q, s \in(0,1)$. If $\left(\mu_{0}, \mu_{1}\right) \in$ $\mathcal{P}_{c}(M)^{2}$ is q-separated and $X_{i}:=\operatorname{spt} \mu_{i}$, there is a continuous map $W$ : $Z_{s}(S) \subset M \longrightarrow S \subset X_{0} \times X_{1}$ such that if $\mu_{s}$ lies on the q-geodesic (8) then $W_{\#} \mu_{s}$ maximizes $\ell^{q}$ in $\Pi\left(\mu_{0}, \mu_{1}\right)$. In fact, the map $W$ is Lipschitz continuous with respect to any fixed choice of Riemannian distance $d_{\tilde{g}}$ on $M$. Here $Z_{s}$ is from (16) and $S$ from the Definition 4.1 of q-separated.

Proof. Fix $(q, s) \in(0,1)$ and let $\left(\mu_{0}, \mu_{1}\right) \in \mathcal{P}_{c}(M)^{2}$ be $q$-separated and $\mu_{s}$ satisfy (8). Setting $X=\operatorname{spt} \mu_{0}$ and $Y:=\operatorname{spt} \mu_{1}$, by Theorem 4.3 there exist potentials $u \oplus v \geq \frac{1}{q} \ell^{q}$ such that the compact set $S:=\{(x, y) \in X \times Y \mid$ $\left.u \oplus v=\frac{1}{q} \ell^{q}\right\}$ is disjoint from $\{\ell \leq 0\}$ and contains the support of one hence all, in view of (33) - maximizers $\pi \in \Pi(\mu, \nu)$ for (6). We claim the map $W: Z \longrightarrow S$ from Corollary 5.2 is Lipschitz with respect to the Riemannian distance $d=d_{\tilde{g}}$, where $Z:=Z_{s}(S)$ is the compact image of $S$ from Lemma 2.5. Equivalently, there exists a constant $C_{s}<\infty$ such that whenever $\left(x^{ \pm}, y^{ \pm}\right)=W\left(z^{ \pm}\right)$with $z^{ \pm} \in Z$,

$$
d\left(x^{+}, x^{-}\right)+d\left(y^{+}, y^{-}\right) \leq C_{s} d\left(z^{+}, z^{-}\right) .
$$

We'll establish this for $s=\frac{1}{2}$ without losing generality.

For each integer $k \in \mathbf{N}$ set

$$
\begin{aligned}
I_{k} & :=\inf _{d\left(z^{+}, z^{-}\right) \geq 1 / k} \frac{d\left(z^{+}, z^{-}\right)}{d\left(x^{+}, x^{-}\right)+d\left(y^{+}, y^{-}\right)} \\
& =\frac{d\left(z_{k}^{+}, z_{k}^{-}\right)}{d\left(x_{k}^{+}, x_{k}^{-}\right)+d\left(y_{k}^{+}, y_{k}^{-}\right)},
\end{aligned}
$$

where the infimum is over pairs $\left(x^{ \pm}, y^{ \pm}\right)=W\left(z^{ \pm}\right)$with $z^{ \pm} \in Z$. Compactness of $Z$ implies $I_{k}$ is attained, positive and non-increasing; our goal is to show that its limit $I_{\infty}$ is also strictly positive. If so, then $C_{s}=1 / I_{\infty}$ is the desired Lipschitz constant.

Use compactness of $Z$ to extract convergent subsequences $z_{k}^{ \pm} \rightarrow z_{\infty}^{ \pm}$; the properties of $W: Z \longrightarrow S$ established in Corollary 5.2 guarantee $\left(x_{k}^{ \pm}, y_{k}^{ \pm}\right) \rightarrow$ $\left(x_{\infty}^{ \pm}, y_{\infty}^{ \pm}\right)$and $z_{\infty}^{ \pm} \in Z_{\frac{1}{2}}\left(x_{\infty}^{ \pm}, y_{\infty}^{ \pm}\right)$along these subsequences. We henceforth assume $d\left(z_{\infty}^{+}, z_{\infty}^{-}\right)=0$, since otherwise we are done. Continuity of $W$ then implies $\left(x_{\infty}^{+}, y_{\infty}^{+}\right)=\left(x_{\infty}^{-}, y_{\infty}^{-}\right)=:\left(x_{\infty}, y_{\infty}\right)$. Let $t \in\left[-\frac{1}{2}, \frac{1}{2}\right] \mapsto \sigma_{k}^{ \pm}(t)=$ $\exp _{z_{k}^{ \pm}}^{g} t v_{k}^{ \pm}$denote the timelike geodesic joining $x_{k}^{ \pm}$to $y_{k}^{ \pm}$passing through $z_{k}^{ \pm}=\sigma_{k}^{ \pm}(0)$. This means $\sigma_{k}^{+}$and $\sigma_{k}^{-}$have the same subsequential limiting 
geodesic $\sigma_{\infty}$. Since $\left(x_{\infty}, y_{\infty}\right) \in S \subset\{\ell>0\}$ this geodesic is timelike: $y_{\infty}$ lies in the chronological future of $x_{\infty}$.

Setting $R_{k}:=d_{T M}\left(\left(v_{k}^{+}, z_{k}^{+}\right),\left(v_{k}^{-}, z_{k}^{-}\right)\right)$yields $r_{k}:=d\left(z_{k}^{+}, z_{k}^{-}\right) \in\left[\frac{1}{k}, R_{k}\right]$ and $R_{k} \rightarrow 0$. Adopting Fermi coordinates along the limiting timelike geodesic $\sigma_{\infty}$, and suppressing the subscripts $k$, for $k$ sufficiently large set $(\Delta v, \Delta z):=$ $\left(v_{k}^{-}-v_{k}^{+}, z_{k}^{-}-z_{k}^{+}\right)$and

$$
\begin{aligned}
J_{k}(t) & :=\frac{1}{R_{k}}\left[\exp _{\sigma^{+}(t)}^{\tilde{g}}\right]^{-1} \sigma^{-}(t) \\
& =\frac{1}{R_{k}}\left(D \exp ^{g}\right)_{\left(t v^{+}, z^{+}\right)}\left(\begin{array}{c}
t \Delta v \\
\Delta z
\end{array}\right)+O\left(R_{k}\right) .
\end{aligned}
$$

Choosing a further subsequence (without relabelling) along which

$$
\lim _{k \rightarrow \infty} \frac{1}{R_{k}}(\Delta z, \Delta v)=\left(\Delta v_{\infty}, \Delta z_{\infty}\right) \in T_{\left(v_{\infty}, z_{\infty}\right)} T M
$$

converges to a vector with unit Riemannian length. Along this subsequence $J_{\infty}(t)=\lim _{k \rightarrow \infty} J_{k}(t)$ converges to a Lorentzian Jacobi field along $\sigma_{\infty}$. This Jacobi field is non-trivial, since $J_{\infty}(0)=\Delta z_{\infty}$, and when $\Delta z_{\infty}=0$ then $J_{\infty}^{\prime}(0)=\Delta v_{\infty}$ has unit Riemannian norm. Although the rate of convergence of (83) can be slow, $\Delta z_{\infty}=0$ implies $r_{k}=o\left(R_{k}\right)$ and

$$
d_{T M}\left(J_{k}\left(c_{k}\right), J_{\infty}\left(c_{k}\right)\right)=o\left(c_{k}\right)+O\left(R_{k}\right) \quad \text { when } \frac{r_{k}}{R_{k}} \ll c_{k},
$$

i.e. as $k \rightarrow \infty$ when $c_{k} \neq 0$ is bounded away from zero or tends to zero more slowly than $r_{k} / R_{k}$.

Now, since $\sigma^{-}(t)=\exp _{\sigma^{+}(t)}^{\tilde{g}} R_{k} J_{k}(t)$ and hence

$$
d\left(\sigma^{+}(t), \sigma^{-}(t)\right)=R_{k}\left|J_{k}(t)\right|_{\tilde{g}}
$$

we find

$$
\begin{aligned}
I_{\infty} & =\lim _{k \rightarrow \infty} \frac{d\left(z_{k}^{+}, z_{k}^{-}\right)}{d\left(x_{k}^{+}, x_{k}^{-}\right)+d\left(y_{k}^{+}, y_{k}^{-}\right)} \\
& =\frac{\left|J_{\infty}(0)\right|_{\tilde{g}}}{\left|J_{\infty}\left(-\frac{1}{2}\right)\right|_{\tilde{g}}+\left|J_{\infty}\left(\frac{1}{2}\right)\right|_{\tilde{g}}} .
\end{aligned}
$$

If $J_{\infty}(0) \neq 0$ the denominator cannot vanish since $I_{\infty} \leq I_{k}<\infty$; in this case we are done. To derive a contradiction, we may therefore assume $J_{\infty}(0)=0$. Then $J_{\infty}^{\prime}(0) \neq 0$ and

$$
J_{\infty}(t)=t J^{\prime}(0)+O\left(t^{3}\right)
$$


as $t \rightarrow 0$. (In fact $o(t)$ would be enough for our purposes: we shall never need the fact that $J_{\infty}$ is a Jacobi field except to guarantee its differentiability at the origin; it is another irrelevant fact that the denominator above cannot vanish since no non-trivial Jacobi field vanishes both at the endpoints and the midpoint of a proper-time maximizing geodesic segment.)

Choose any decaying sequence of times $c_{k} \gg \max \left\{\frac{r_{k}}{R_{k}}, R_{k}\right\}$. For large $k$, fixed and tacit, $a, b \in[0,1]$ and $c>0$ sufficiently small, Riemannian geodesics

$$
\begin{aligned}
x(a) & :=\exp _{\sigma^{+}(-c)}^{\tilde{g}}\left[a R J_{k}(-c)\right] \\
y(b) & :=\exp _{\sigma^{+}(+c)}^{\tilde{g}}\left[b R J_{k}(+c)\right]
\end{aligned}
$$

can be defined so that $x(\cdot)$ interpolates between $\sigma^{ \pm}(-c)$ while $y(\cdot)$ interpolates between $\sigma^{ \pm}(c)$. From (84)-(85) these geodesics have length $O(c R)$ much smaller than the time separation $O(c)$ between their endpoints, hence $k$ large enough implies $y(b)$ lies in the chronological future of $x(a)$ for all $a, b \in[0,1]$. Recalling Theorem 3.6(c), introduce the smooth function

$$
f(a, b):=\frac{1}{q} \ell^{q}(x(a), y(b))
$$

where both $x$ and $y$ depend implicitly on $k$. Since $\left(x_{k}^{ \pm}, y_{k}^{ \pm}\right) \in S$, the $\ell^{q_{-}}$ monotonicity of $S$ established in Theorem 4.3 implies

$$
0 \leq f(0,0)+f(1,1)-f(0,1)-f(1,0)=\int_{0}^{1} \int_{0}^{1} \frac{\partial^{2} f}{\partial a \partial b} d a d b
$$

holds for $c=\frac{1}{2}$; in fact it holds also for each $c \in\left[0, \frac{1}{2}\right]$ by the same theorem applied to the support of $\ell^{q}$-optimal measure $\left(z_{\frac{1}{2}-c} \times z_{\frac{1}{2}+c}\right)_{\#} \pi$ from Theorem 2.11. Always assuming $J_{\infty}(0)=0$, we'll derive a contradiction to this conclusion by showing the mixed partials of $f$ are negative for $k$ sufficiently large. Let $\gamma:=\gamma_{(a, b)}:[0,1] \longrightarrow M$ denote the proper-time maximizing geodesic connecting $x(a)$ to $y(b)$. From

$$
f(a, b)=-\int_{0}^{1} L\left(\dot{\gamma}_{(a, b)}(t) ; q\right) d t=-\frac{1}{q} \int_{0}^{1}\left|\dot{\gamma}_{(a, b)}(t)\right|^{q} d t
$$

we compute

$$
\begin{aligned}
-\frac{\partial^{2} f}{\partial a \partial b} & =\int_{0}^{1}\left[D^{2} L\left(\frac{\partial \dot{\gamma}}{\partial a}, \frac{\partial \dot{\gamma}}{\partial b}\right)+D L \frac{\partial^{2} \dot{\gamma}}{\partial a \partial b}\right] d t \\
& =\int_{0}^{1} D^{2} L\left(\frac{\partial \dot{\gamma}}{\partial a}, \frac{\partial \dot{\gamma}}{\partial b}\right) d t
\end{aligned}
$$


where the $D L$ integral vanishes (after integrating by parts) by the geodesy of $\gamma=\gamma_{(a, b)}$, and the fact that each of its endpoints $\gamma_{(a, b)}(0)=x(a)$ and $\gamma_{(a, b)}(1)=y(b)$ depend only on one of the two variables $a$ and $b$.

Recall that the Lorentzian geodesic $\gamma_{(a, b)}$ depends smoothly on its endpoints, which lie at distance $O(c R)$ from those of $\gamma_{(0,0)}$. Observe that $\frac{\partial \gamma}{\partial a}$ and $\frac{\partial \gamma}{\partial b}$ are both Jacobi fields along $\gamma_{(a, b)}$. Moreover $(a, b)=(0,0)$ implies $\frac{\partial \gamma}{\partial a}$ is the Jacobi field with end vectors $R J_{k}(-c)$ and 0 , while $\frac{\partial \gamma}{\partial b}$ has end vectors 0 and $R J_{k}(c)$. Since the geodesics in question are collapsing to a point where the geometry is asymptotic to Minkowski space, these Jacobi fields are asympotically linear. The intermediate value theorem and Jacobi equation yield

$$
\begin{aligned}
\frac{\partial \dot{\gamma}_{(a, b)}}{\partial a} & =\frac{-R J_{k}(-c)}{2 c \ell\left(x_{k}^{+}, y_{k}^{+}\right)}+O(c R) \\
\frac{\partial \dot{\gamma}_{(a, b)}}{\partial b} & =\frac{R J_{k}(c)}{2 c \ell\left(x_{k}^{+}, y_{k}^{+}\right)}+O(c R) .
\end{aligned}
$$

From (84)-

$$
\frac{\partial \dot{\gamma}_{(a, b)}}{\partial a}=\frac{R J_{\infty}^{\prime}(0)}{2 \ell\left(x_{\infty}, y_{\infty}\right)}+o(R)=\frac{\partial \dot{\gamma}_{(a, b)}}{\partial b}
$$

The positive-definiteness (Lemma 3.1) of $D^{2} L$ in (87) at $\dot{\gamma}_{(a, b)}(t)=\dot{\gamma}_{0,0}(t)+$ $O(c R)$ gives the desired contradiction $\frac{\partial^{2} f}{\partial a \partial b}(a, b)<0$ to (86) for all $a, b \in[0,1]$ and $k$ sufficiently large, thus establishing the theorem.

\section{References}

[1] A. Agrachev and P.W.Y. Lee. Optimal transportation under nonholonomic constraints. Trans. Amer. Math. Soc. 361, 6019-6047 (2009).

[2] N. Ahmad, H.K. Kim, and R.J. McCann. Optimal transportation, topology and uniqueness. Bull. Math. Sci. 1, 13-32 (2011).

[3] A.D. Aleksandrov. Almost everywhere existence of the second differential of a convex function and some properties of convex surfaces connected with it (In Russian). Uchen. Zap. Leningrad. Gos. Univ., Math. Ser. 6, 3-35 (1939). 
[4] C.D. Aliprantis and K.C. Border. Infinite dimensional analysis. A hitchhiker's guide. Third edition. Springer, Berlin, (2006)

[5] L. Ambrosio and S. Rigot. Optimal transportation in the Heisenberg group. J. Funct. Anal. 208, 261-301 (2004).

[6] L. Ambrosio, N. Fusco, and D. Pallara. Functions of bounded variation and free discontinuity problems. The Clarendon Press, Oxford University Press, New York, (2000).

[7] L. Ambrosio and N. Gigli. A user's guide to optimal transport. In Modelling and optimisation of flows on networks, volume 2062 of Lecture Notes in Math., pages 1-155. Springer, Heidelberg (2013).

[8] L. Ambrosio, N. Gigli, A. Mondino, and T. Rajala. Riemannian Ricci curvature lower bounds in metric measure spaces with $\sigma$-finite measure. Trans. Amer. Math. Soc. 367, 4661-4701 (2015).

[9] L.A. Ambrosio, N. Gigli, and G. Savaré. Gradient flows in metric spaces and in the space of probability measures. Lecture Notes in Mathematics ETH Zürich. Birkhäuser Verlag, Basel (2005).

[10] L. Ambrosio, N. Gigli, and G. Savaré. Calculus and heat flow in metric measure spaces and applications to spaces with Ricci bounds from below. Invent. Math. 195, 289-391 (2014).

[11] L. Ambrosio, N. Gigli, and G. Savaré. Metric measure spaces with Riemannian Ricci curvature bounded from below. Duke Math. J. 163, 1405-1490 (2014).

[12] L. Ambrosio, N. Gigli, and G. Savaré. Bakry-Émery curvaturedimension condition and Riemannian Ricci curvature bounds. Ann. Probab. 43, 339-404 (2015).

[13] A. Avez. Essais de géométrie riemannienne hyperbolique globale. Applications à la relativité générale. Ann. Inst. Fourier (Grenoble) 13, 105-190 (1963).

[14] K. Bacher and K.T. Sturm. Localization and tensorization properties of the curvature-dimension condition for metric measure spaces. J. Funct. Anal. 259, 28-56 (2010). 
[15] D. Bakry and M. Émery. Diffusions hypercontractives. In Séminaire de probabilités, XIX, 1983/84, volume 1123 of Lecture Notes in Math., pages 177-206. Springer, Berlin (1985).

[16] Z.M. Balogh, A. Kristály, and K. Sipos. Geometric inequalities on Heisenberg groups. Calc. Var. Partial Differential Equations 57, Art. 61, 41 (2018).

[17] V. Bangert. Analytische Eigenschaften konvexer Funktionen auf Riemannschen Mannigfaltigkeiten. J. Reine Angew. Math. 307/308, 309324 (1979).

[18] J.M. Bardeen, B. Carter, and S.W. Hawking. The four laws of black hole mechanics. Comm. Math. Phys. 31, 161-170 (1973).

[19] J.K. Beem, P.E. Ehrlich, and K.L. Easley. Global Lorentzian Geometry. Marcel Dekker, Inc., New York, second edition, (1996).

[20] M. Beiglböck, M. Goldstern, G. Maresch, and W. Schachermayer. Optimal and better transport plans. J. Funct. Anal. 256, 1907-1927 (2009).

[21] J.D. Bekenstein. Black holes and entropy. Phys. Rev. D (3) 7, 2333-2346 (1973).

[22] J.-D. Benamou and Y. Brenier. A computational fluid mechanics solution to the Monge-Kantorovich mass transfer problem. Numer. Math. 84, 375-393 (2000).

[23] A.N. Bernal and M. Sánchez. Globally hyperbolic spacetimes can be defined as 'causal' instead of 'strongly causal'. Classical and Quantum Gravity 24, 745-749 (2007).

[24] P. Bernard and B. Buffoni. The Monge problem for supercritical Mañé potentials on compact manifolds. Adv. Math. 207, 691-706 (2006).

[25] P. Bernard and B. Buffoni. Optimal mass transportation and Mather theory. J. Eur. Math. Soc. (JEMS) 9, 85-121 (2007).

[26] J. Bertrand, A. Pratelli, and M. Puel. Kantorovich potentials and continuity of total cost for relativistic cost functions. J. Math. Pures Appl. (9) 110, 93-122 (2018). 
[27] J. Bertrand and M. Puel. The optimal mass transport problem for relativistic costs. Calc. Var. Partial Differential Equations 46, 353-374 (2013).

[28] Y. Brenier. Polar factorization and monotone rearrangement of vectorvalued functions. Comm. Pure Appl. Math. 44, 375-417 (1991).

[29] Y. Brenier. Extended Monge-Kantorovich theory. In Optimal transportation and applications (Martina Franca, 2001), volume 1813 of Lecture Notes in Math., pages 91-121. Springer, Berlin (2003).

[30] Y. Brenier, U. Frisch, M. Hénon, G. Loeper, S. Matarrese, R. Moyahee, and A. Sobolevskii. Reconstruction of the early Universe as a convex optimization problem. Mon. Not. R. Astron. Soc. 346, 501524 (2003).

[31] S. Carroll. Spacetime and geometry. An introduction to general relativity. San Francisco: Addison Wesley, 2004.

[32] J.S. Case. Singularity theorems and the Lorentzian splitting theorem for the Bakry-Emery-Ricci tensor. J. Geom. Phys. 60, 477-490 (2010).

[33] F. Cavalletti and E. Milman. The globalization theorem for the curvature dimension condition. Preprint at arxiv.org/abs/1612.07623.

[34] F. Cavalletti and A. Mondino. Sharp and rigid isoperimetric inequalities in metric-measure spaces with lower Ricci curvature bounds. Invent. Math. 208, 803-849 (2017).

[35] F. Cavalletti and A. Mondino. Sharp geometric and functional inequalities in metric measure spaces with lower Ricci curvature bounds. Geom. Topol. 21, 603-645 (2017).

[36] D. Cordero-Erausquin, R.J. McCann and M. Schmuckenschläger. A Riemannian interpolation inequality à la Borell, Brascamp and Lieb. Invent. Math. 146, 219-257 (2001).

[37] D. Cordero-Erausquin, R.J. McCann and M. Schmuckenschläger. Prekopa-Leindler type inequalities on Riemannian manifolds, Jacobi fields, and optimal transport. Ann. Fac. Sci. Toulouse Math. (6) 15, 613-635 (2006). 
[38] M. Eckstein and T. Miller. Causality for nonlocal phenomena. Ann. Henri Poincaré 18, 3049-3096 (2017).

[39] M. Erbar, K. Kuwada, and K.-T. Sturm. On the equivalence of the entropic curvature-dimension condition and Bochner's inequality on metric measure spaces. Invent. Math. 201, 993-1071 (2015).

[40] L.C. Evans and R.F. Gariepy. Measure Theory and Fine Properties of Functions. Stud. Adv. Math. CRC Press, Boca Raton, (1992).

[41] A. Fathi and A. Figalli. Optimal transportation on non-compact manifolds. Israel J. Math. 175, 1-59 (2010).

[42] A. Figalli and L. Rifford. Mass transportation on sub-Riemannian manifolds. Geom. Funct. Anal. 20, 124-159 (2010).

[43] U. Frisch, S. Matarrese, R. Moyahee, and A. Sobolevskii. A reconstruction of the initial conditions of the Universe by optimal mass transportation. Nature 417, 260-262 (2002).

[44] W. Gangbo and R.J. McCann The Geometry of Optimal Transportation. Acta Math. 177,"113-161 (1996).

[45] R. Geroch. Spinor structure of space-times in general relativity. I. J. Mathematical Phys. 9, 1739-1744 (1968).

[46] N. Gigli. Optimal maps in non branching spaces with Ricci curvature bounded from below. Geom. Funct. Anal. 22, 990-999 (2012).

[47] N. Gigli. An overview of the proof of the splitting theorem in spaces with non-negative Ricci curvature. Anal. Geom. Metr. Spaces 2, 169-213 (2014).

[48] D.A. Gomes and T. Senici. Displacement convexity for first-order mean field games. Preprint at axXiv:180\%.07090.

[49] N. Gigli, T. Rajala, and K.-T. Sturm. Optimal maps and exponentiation on finite-dimensional spaces with Ricci curvature bounded from below. J. Geom. Anal. 26, 2914-2929 (2016).

[50] R. Haslhofer and A. Naber. Ricci curvature and Bochner Formulas for Martingales. Preprint at axXiv:1608.04371. 
[51] S.W. Hawking. The occurrence of singularities in cosmology. I. Proc. Roy. Soc. Ser. A 294, 511-521 (1966).

[52] S.W. Hawking and G.F.R. Ellis. The large scale structure of space-time. Cambridge University Press, London-New York (1973).

[53] S.W. Hawking and R. Penrose. The singularities of gravitational collapse and cosmology. Proc. Roy. Soc. London Ser. A 314, 529-548 (1970).

[54] T. Jacobson. Thermodynamics of spacetime: the Einstein equation of state. Phys. Rev. Lett. 75, 1260-1263 (1995).

[55] M. Kell. On interpolation and curvature via Wasserstein geodesics. Adv. Calc. Var. 10, 125-167 (2017).

[56] M. Kell and S. Suhr. On the existence of dual solutions for Lorentzian cost functions. Preprint at arXiv:1808.04393v1.

[57] C. Ketterer. Obata's rigidity theorem for metric measure spaces. Anal. Geom. Metr. Spaces 3, 278-295 (2015).

[58] M. Kunzinger, and C. Sämann. Lorentzian length spaces. Preprint at arXiv:1711.08990v1.

[59] P.W.Y. Lee. Displacement interpolations from a Hamiltonian point of view. J. Funct. Anal. 265, 3163-3203 (2013).

[60] P.W.Y. Lee, C. Li, and I. Zelenko. Ricci curvature type lower bounds for sub-Riemannian structures on Sasakian manifolds. Discrete Contin. Dyn. Syst. 36, 303-321 (2016).

[61] G. Loeper. The reconstruction problem for the Euler-Poisson system in cosmology. Arch. Ration. Mech. Anal. 179, 153-216 (2006).

[62] J. Lott and C. Villani. Ricci curvature for metric measure spaces via optimal transport. Annals Math. (2) 169, 903-991, (2009).

[63] J.N. Mather. Action minimizing invariant measures for positive definite Lagrangian systems. Math. Z. 207, 169-207 (1991).

[64] R.J. McCann. A Convexity Theory for Interacting Gases and Equilibrium Crystals. PhD thesis, Princeton University (1994). 
[65] R.J. McCann. Existence and uniqueness of monotone measurepreserving maps. Duke Math. J. 80, 309-323 (1995).

[66] R.J. McCann. A convexity principle for interacting gases. Adv. Math. 128, 153-179 (1997).

[67] R.J. McCann. Polar factorization of maps on Riemannian manifolds. Geom. Funct. Anal. 11, 589-608 (2001).

[68] R.J. McCann, B. Pass and M. Warren. Rectifiability of optimal transportation plans. Canad. J. Math. 64, 924-934 (2012).

[69] R.J. McCann and M. Puel. Constructing a relativistic heat flow by time transport steps. Ann. Inst. H. Poincare Anal. Non Lineaire 26, 2539-2580 (2009).

[70] A. Mondino and A. Naber. Structure theory of metric-measure spaces with lower Ricci curvature bounds. To appear in J. Euro. Math. Soc.

[71] G. Monge. Mémoire sur la théorie des déblais et de remblais. Histoire de l'Académie Royale des Sciences de Paris, avec les Mémoires de Mathématique et de Physique pour la même année, pages 666-704 (1781).

[72] A. Naber. Characterizations of bounded Ricci curvature on smooth and non-smooth spaces. Preprint at arXiv:1306.6512.

[73] K. Nomizu and H. Ozeki. The existence of complete Riemannian metrics. Proc. Amer. Math. Soc. 12, 889-891 (1961).

[74] S. Ohta, Finsler interpolation inequalities. Calc. Var. Partial Differential Equations 36, 211-249 (2009)

[75] S. Ohta. On the curvature and heat flow on Hamiltonian systems. Anal. Geom. Metr. Spaces 2, 81-114 (2014).

[76] S. Ohta. Splitting theorems for Finsler manifolds of nonnegative Ricci curvature. J. Reine Angew. Math. 700, 155-174 (2015).

[77] B. O'Neill. Semi-Riemannian Geometry (with applications to Relativity). Academic Press, San Diego (1983). 
[78] F. Otto and C. Villani. Generalization of an inequality by Talagrand and links with the logarithmic Sobolev inequality. J. Funct. Anal. 173, 361-400 (2000).

[79] R. Penrose. Gravitational collapse and space-time singularities. Phys. Rev. Lett. 14, 57-59 (1965).

[80] M.-K. von Renesse and K.-T. Sturm. Transport inequalities, gradient estimates, entropy and Ricci curvature. Comm. Pure Appl. Math. 58, 923-940 (2005).

[81] R.T. Rockafellar. Convex Analysis. Princeton University Press, Princeton (1970).

[82] F. Santambrogio. Optimal transport for applied mathematicians. Birkhäuser/Springer, Cham (2015).

[83] B. Schachter. An Eulerian Approach to Optimal Transport with Applications to the Otto Calculus. PhD thesis, University of Toronto (2017).

[84] H.-J. Seifert. Global connectivity by timelike geodesics. Z. Naturforsch 22a, 1356-1360 (1967).

[85] K.-T. Sturm. On the geometry of metric measure spaces, I and II. Acta Math. 196, 65-177 (2006).

[86] K.-T. Sturm. Super-Ricci flows for metric measure spaces. Preprint at arXiv:1603.02193

[87] K.-T. Sturm. Remarks about synthetic upper Ricci bounds for metric measure spaces. Preprint at arXiv:1711.01707

[88] S. Suhr. Theory of optimal transport for Lorentzian cost functions. Preprint at arXiv:1601.04532. To appear in Münster J. Math.

[89] J.-H. Treude and J.D.E. Grant. Volume comparison for hypersurfaces in Lorentzian manifolds and singularity theorems. Ann. Global Anal. Geom. 43, 233-251 (2013).

[90] E. Verlinde. On the origin of gravity and the laws of newton. J. High Energy Phys. 2011, 029 (2011). 
[91] E. Verlinde. Emergent gravity and the dark universe. SciPost Phys. 2 016 (2017).

[92] C. Villani. Optimal Transport. Old and New. Springer, New York (2009).

[93] R.M. Wald. General Relativity. The University of Chicago Press, Chicago (1984).

[94] E. Woolgar and W. Wylie. Cosmological singularity theorems and splitting theorems for $\mathrm{N}$-Bakry-Émery spacetimes. J. Math. Phys., 57 022504, 12 (2016). 